\title{
ASYMMETRIC DECARBOXYLATIVE ALLYLATION OF OXINDOLES
}

Vilius Franckevičius, James D. Cuthbertson, Mark Pickworth, David S. Pugh and Richard J. K. Taylor

Department of Chemistry, University of York, Heslington, York YO10 5DD, UK E-mail: richard.taylor@york.ac.uk

\section{SUPPORTING INFORMATION}

1. Catalyst and Solvent Screen S-2

2. General Experimental Section S-3

3. Experimental Section S-5

3.1. Monoesterification of Malonic Acid $\quad$ S-5

3.2. Anilide Formation from B-Carbonyl Acids S-7

3.3. Dicarbonyl Alkylation $\quad$ S-10

3.4. Anilide Formation from Phenylacetic Acids S-15

3.5. Allyloxycarbonylation of Phenylacetamides S-18

3.6. Oxindole Cyclization S-21

3.7. Decarboxylative Allyl Transfer S-27

4. Comparison of Optical Rotation Data $\quad$ S-34

5. References $\quad$ S-35

6. HPLC Data $\quad$ S-36

7. ${ }^{1} \mathrm{H}$ NMR Spectra $\quad$ S-47 
<smiles>[R]C1(C(=O)OCC=C)C(=O)Nc2ccccc21</smiles>

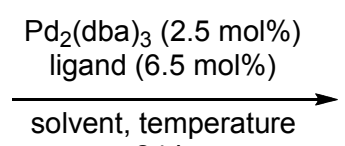

$24 \mathrm{~h}$

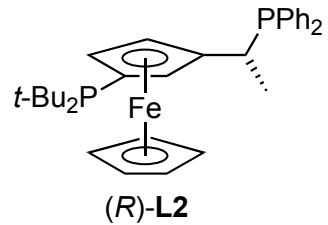

$(R)-\mathrm{L} 1$<smiles>CCC(C)[C@H]1COC(c2ccccc2-c2ccccc2)=N1</smiles>

(R)-L2<smiles></smiles>

6h: $R=P h$<smiles>Pc1ccccc1-c1ccc2ccccc2c1-c1c(P)ccc2ccccc12</smiles>

(R)-L3

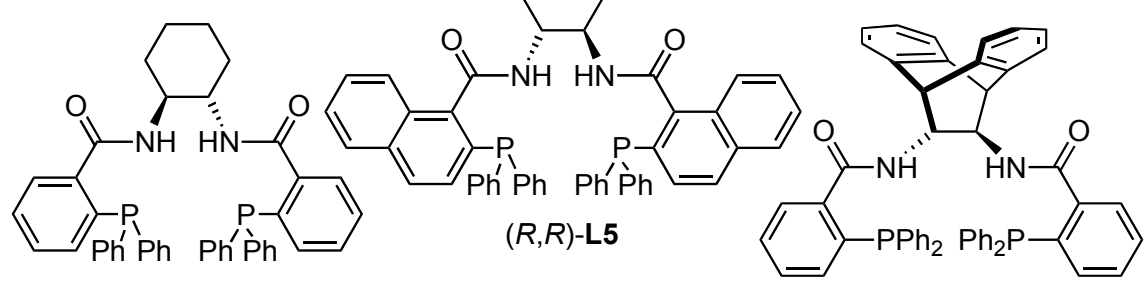

$(S, S)$-L4 $(R, R)-\mathrm{L} 6$

\begin{tabular}{|c|c|c|c|c|c|c|}
\hline entry & $\mathrm{R}$ & solvent & temp. $\left({ }^{\circ} \mathrm{C}\right)$ & ligand & yield $(\%)^{a}$ & ee $(\%)^{k}$ \\
\hline 1 & $\mathrm{Me}$ & THF & rt & $(R)-\mathrm{L} 1$ & 82 & 4 \\
\hline 2 & $\mathrm{Me}$ & THF & $\mathrm{rt}$ & $(R)-\mathrm{L} 2$ & 92 & -6 \\
\hline 3 & Me & THF & $\mathrm{rt}$ & (R)-L3 & 95 & -14 \\
\hline 4 & $\mathrm{Me}$ & THF & rt & $(S, S)-L 4$ & 32 & 4 \\
\hline 5 & Me & THF & $\mathrm{rt}$ & $(R, R)-\mathbf{L} 5$ & 10 & -12 \\
\hline 6 & $\mathrm{Me}$ & 1,4-dioxane & rt & $(R, R)-\mathbf{L} 6$ & 0 & - \\
\hline 7 & $\mathrm{Me}$ & toluene & rt & $(R, R)-\mathbf{L} 6$ & 66 & 11 \\
\hline 8 & Me & 1,2-DCE & $\mathrm{rt}$ & $(R, R)-\mathrm{L} 6$ & 97 & 11 \\
\hline 9 & $\mathrm{Me}$ & $\mathrm{Et}_{2} \mathrm{O}$ & $\mathrm{rt}$ & $(R, R)-\mathbf{L} 6$ & 52 & 18 \\
\hline 10 & $\mathrm{Me}$ & $\mathrm{CH}_{2} \mathrm{Cl}_{2}$ & rt & $(R, R)$-L6 & 90 & 37 \\
\hline 11 & $\mathrm{Me}$ & THF & rt & $(R, R)-\mathbf{L} 6$ & 73 & 46 \\
\hline 12 & $\mathrm{Me}$ & DME & rt & $(R, R)-\mathbf{L} 6$ & 92 & 52 \\
\hline 13 & Me & THF & -40 & $(R, R)-\mathrm{L} 6$ & 85 & 57 \\
\hline 14 & Me & DME & -25 & $(R, R)-\mathrm{L} 6$ & 83 & 74 \\
\hline 15 & $\mathrm{Me}$ & DME & -40 & $(R, R)$-L6 & 0 & - \\
\hline 16 & $\mathrm{Ph}$ & toluene & $\mathrm{rt}$ & $(R)-\mathbf{L} 1$ & 99 & -9 \\
\hline 17 & $\mathrm{Ph}$ & toluene & $\mathrm{rt}$ & $(R)-\mathrm{L} 2$ & 42 & 28 \\
\hline 18 & $\mathrm{Ph}$ & toluene & rt & $(R)-\mathrm{L} 3$ & 99 & 19 \\
\hline 19 & $\mathrm{Ph}$ & toluene & rt & $(S, S)-L 4$ & 91 & -7 \\
\hline 20 & $\mathrm{Ph}$ & toluene & rt & $(R, R)-\mathbf{L} 5$ & 93 & 7 \\
\hline 21 & $\mathrm{Ph}$ & toluene & rt & $(R, R)-\mathbf{L} 6$ & quant. & 64 \\
\hline 22 & $\mathbf{P h}$ & toluene & -25 & $(R, R)-\mathrm{L} 6$ & 99 & 79 \\
\hline 23 & $\mathrm{Ph}$ & DME & -25 & $(R, R)-\mathbf{L} 6$ & 84 & 52 \\
\hline 24 & $\mathrm{Ph}$ & THF & -25 & $(R, R)-\mathbf{L} 6$ & 96 & 43 \\
\hline
\end{tabular}

${ }^{a}$ Isolated yield; ${ }^{b}$ determined by chiral HPLC. 


\section{General Experimental Section}

All reactions were performed in oven-dried glassware under an atmosphere of argon unless specified otherwise. Acetonitrile, dichloromethane, tetrahydrofuran, diethyl ether and toluene were dried on an Innovative Technology Pure Solv solvent purification system by passing the solvent through activated alumina and copper catalyst columns, as appropriate. All other reagents and solvents were used without further purification from commercial sources. Petrol refers to the fractions of petroleum ether which boil between 40 and $60^{\circ} \mathrm{C}$. Aqueous solutions are saturated unless specified otherwise.

Flash column chromatography was carried out using Merck Kieselgel (40-63 $\mu$ m particle size). Analytical thin layer chromatography (TLC) was performed using pre-coated plastic-backed plates (Fluka Kieselgel 60 F254), and visualised by ultra-violet irradiation $(254 \mathrm{~nm})$ and by staining with aqueous acidic ceric ammonium molybdate(IV) or potassium permanganate solutions as appropriate.

${ }^{1} \mathrm{H}$ NMR spectra were recorded on a Jeol ECX-400 NMR spectrometer and are reported as follows: chemical shift, $\delta_{H}$ (in parts per million, ppm), (multiplicity, coupling constant, $J$, and number of protons). Couplings are expressed as: s, singlet; $d$, doublet; t, triplet; q, quartet; sept, septuplet; m, multiplet; br, broad or combinations of these. Where coincident coupling constants have been observed in the NMR spectrum, the apparent multiplicity of the proton resonance concerned is reported. For spectra recorded in $\mathrm{CDCl}_{3}$, the central resonance of residual protic solvents $\mathrm{CHCl}_{3}\left(\delta_{\mathrm{H}}=7.26 \mathrm{ppm}\right)$ was used as the internal reference. ${ }^{13} \mathrm{C}$ NMR spectra were recorded on the same spectrometer at $100 \mathrm{MHz}$, using the central resonance of $\mathrm{CDCl}_{3}\left(\delta_{\mathrm{C}}=77.0 \mathrm{ppm}\right)$ as the internal reference.

Melting points were measured on a Gallenkamp melting point apparatus and are uncorrected.

IR spectra were recorded on a Jasco FT/IR-4100 spectrometer. Samples were prepared as a neat film. 
Accurate mass data were recorded using a Bruker Daltronics microOTOF spectrometer by electrospray ionisation (ESI) at the Department of Chemistry, University of York, York, UK.

Optical rotation analysis was performed using a Jasco DIP-370 polarimeter using a sodium lamp ( $\lambda 589 \mathrm{~nm}$, D-line); $[\mathrm{a}]_{D}{ }^{25}$ values are reported in $10^{-1} \mathrm{deg} \mathrm{cm}^{2} \mathrm{~g}^{-1}$; concentration $(c)$ is in $\mathrm{g}$ per $100 \mathrm{~mL}$.

Enantiomeric excesses were determined by HPLC on an Agilent 1200 series HPLC system. 


\section{Experimental Section}

\subsection{Monoesterification of Malonic Acid}

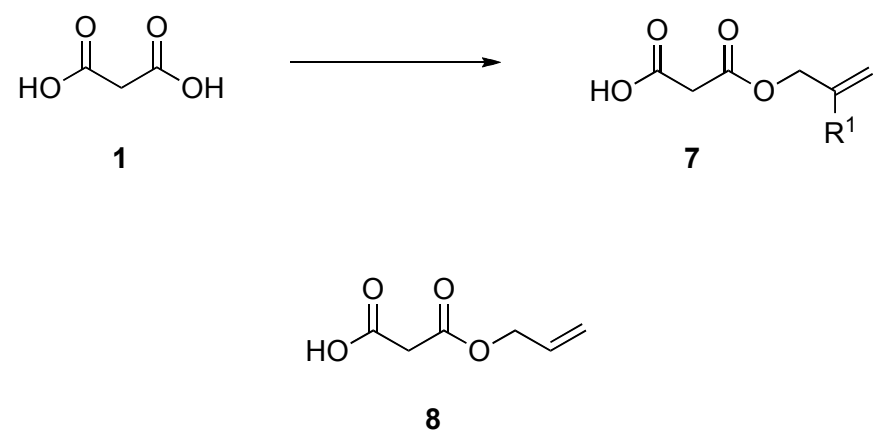

3-(Allyloxy)-3-oxopropanoic acid (8). To a solution of malonic acid (1, $5.20 \mathrm{~g}, 50 \mathrm{mmol}$ ) and allyl alcohol $(6.8 \mathrm{~mL}, 100 \mathrm{mmol})$ in acetonitrile $(150 \mathrm{~mL})$ was added dropwise a suspension of DCC $(11.35 \mathrm{~g}, 55 \mathrm{mmol})$ in acetonitrile $(50 \mathrm{~mL})$. The resulting mixture was stirred at room temperature for $30 \mathrm{~min}$, then filtered and concentrated in vacuo. The residue was dissolved in $\mathrm{Et}_{2} \mathrm{O}(250 \mathrm{~mL})$ and washed with aq. $\mathrm{NaHCO}_{3}(2 \times 100 \mathrm{~mL})$. The combined aqueous phase was acidified to $\mathrm{pH} 1$ with aq. $\mathrm{HCl}(10 \%, 250 \mathrm{~mL})$ and extracted with EtOAc $(2 \times 200 \mathrm{~mL})$. The combined organic fractions were washed with brine (300 $\mathrm{mL})$, dried $\left(\mathrm{MgSO}_{4}\right)$ and concentrated in vacuo to afford crude $8(5.23 \mathrm{~g}, 73 \%)$ as a yellow oil, which was identical to previously isolated material, ${ }^{1}$ and used in the next step without further purification. $R_{F} 0.31$ [Petrol:EtOAc 1:1]; $v_{\max }(\mathrm{film}) / \mathrm{cm}^{-1} 3491,3089,2951,2666$, $1741 ; \delta_{\mathrm{H}}\left(400 \mathrm{MHz}, \mathrm{CDCl}_{3}\right) 10.58$ (br s, $\left.1 \mathrm{H}\right), 5.91$ (ddt, $J=16.4,10.6,5.8 \mathrm{~Hz}, 1 \mathrm{H}$ ), 5.34 (dq, $J=16.5,1.5 \mathrm{~Hz}, 1 \mathrm{H}$ ), $5.26(\mathrm{dq}, J=10.4,1.2 \mathrm{~Hz}, 1 \mathrm{H}), 4.66(\mathrm{dt}, J=5.8,1.3 \mathrm{~Hz}, 2 \mathrm{H}$ ), 3.47 (s, 2H); $\delta_{\mathrm{C}}\left(100 \mathrm{MHz}, \mathrm{CDCl}_{3}\right)$ 171.9, 166.2, 131.1, 119.1, 66.4, 40.9; HRMS (ESI) Found: $[\mathrm{M}+\mathrm{Na}]^{+}, 167.0315 . \mathrm{C}_{6} \mathrm{H}_{8} \mathrm{O}_{4}$ requires $[\mathrm{M}+\mathrm{Na}]^{+}, 167.0315$.

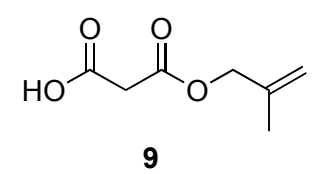

3-(2-Methylallyloxy)-3-oxopropanoic acid (9). To a solution of malonic acid (1, $1.04 \mathrm{~g}$, $10 \mathrm{mmol})$ and 2-methyl-2-propen-1-ol $(1.68 \mathrm{~mL}, 20 \mathrm{mmol})$ in acetonitrile $(30 \mathrm{~mL})$ was added dropwise a suspension of DCC $(2.27 \mathrm{~g}, 11 \mathrm{mmol})$ in acetonitrile $(10 \mathrm{~mL})$. The resulting mixture was stirred at room temperature for $1 \mathrm{~h}$, then filtered and concentrated in vacuo. The residue was dissolved in $\mathrm{Et}_{2} \mathrm{O}(100 \mathrm{~mL})$ and washed with aq. $\mathrm{NaHCO}_{3}(2 \times 100$ $\mathrm{mL})$. The combined aqueous phase was acidified to $\mathrm{pH} 1$ with aq. $\mathrm{HCl}(10 \%, 250 \mathrm{~mL})$ and 
extracted with EtOAc $(2 \times 200 \mathrm{~mL})$. The combined organic fractions were washed with brine $(300 \mathrm{~mL})$, dried $\left(\mathrm{MgSO}_{4}\right)$ and concentrated in vacuo to afford crude $9(1.01 \mathrm{~g}, 64 \%)$ as a clear oil, which was used in the next step without further purification. $R_{F} 0.61$ [Petrol:EtOAc 1:1]; $v_{\max }(\mathrm{film}) / \mathrm{cm}^{-1} 2979,1714 ; \delta_{\mathrm{H}}\left(400 \mathrm{MHz}, \mathrm{CDCl}_{3}\right)$ 5.01-4.98 $(\mathrm{m}, 1 \mathrm{H})$, 4.96-4.93 (m, $1 \mathrm{H}), 4.58(\mathrm{~s}, 2 \mathrm{H}), 3.48(\mathrm{~s}, 2 \mathrm{H}), 1.76-1.74(\mathrm{~m}, 3 \mathrm{H}) ; \delta_{\mathrm{C}}\left(100 \mathrm{MHz}, \mathrm{CDCl}_{3}\right)$ 171.9, 166.2, 139.0, 113.8, 69.0, 40.9, 19.3; HRMS (ESI) Found: $[M+H]^{+}, 159.0650$. $\mathrm{C}_{7} \mathrm{H}_{10} \mathrm{O}_{4}$ requires $[\mathrm{M}+\mathrm{H}]^{+}, 159.0652$.

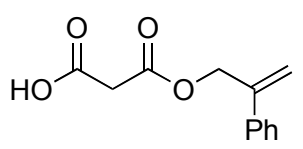

10

3-0xo-3-(2-phenylallyloxy)propanoic acid (10). (The corresponding allylic alcohol 2phenylprop-2-en-1-ol was prepared via a literature procedure. $\left.{ }^{2}\right)$ To a solution of malonic acid (1, $869 \mathrm{mg}, 8.35 \mathrm{mmol})$ and 2-phenylprop-2-en-1-ol (1.68 g, $12.53 \mathrm{mmol})$ in acetonitrile $(30 \mathrm{~mL})$ was added dropwise a suspension of DCC $(1.89 \mathrm{~g}, 9.19 \mathrm{mmol})$ in acetonitrile $(10 \mathrm{~mL})$. The resulting mixture was stirred at room temperature for $1 \mathrm{~h}$, then filtered and concentrated in vacuo. The residue was dissolved in $\mathrm{Et}_{2} \mathrm{O}(100 \mathrm{~mL})$ and washed with aq. $\mathrm{NaHCO}_{3}(2 \times 100 \mathrm{~mL})$. The combined aqueous phase was acidified to $\mathrm{pH}$ 1 with aq. $\mathrm{HCl}(10 \%, 250 \mathrm{~mL})$ and extracted with EtOAc $(2 \times 200 \mathrm{~mL})$. The combined organic fractions were washed with brine $(300 \mathrm{~mL})$, dried $\left(\mathrm{MgSO}_{4}\right)$ and concentrated in vacuo to afford crude $10(1.34 \mathrm{~g}, 73 \%)$ as a pale yellow oil, which was used in the next step without further purification. $R_{F} 0.28$ [Petrol:EtOAc 2:1]; $v_{\max }\left(\right.$ film) $/ \mathrm{cm}^{-1} 2980,1714 ; \delta_{H}$ (400 MHz, $\left.\mathrm{CDCl}_{3}\right)$ 7.45-7.40 (m, 2H), 7.39-7.29 (m, 3H), $5.60(\mathrm{~s}, 1 \mathrm{H}), 5.41(\mathrm{~d}, J=0.9 \mathrm{~Hz}$, $1 \mathrm{H}), 5.09(\mathrm{~d}, J=0.9 \mathrm{~Hz}, 2 \mathrm{H}), 3.46(\mathrm{~s}, 2 \mathrm{H}) ; \delta_{\mathrm{C}}\left(100 \mathrm{MHz}, \mathrm{CDCl}_{3}\right)$ 171.8, 166.2, 141.6, 137.6, 128.5, 128.1, 125.9, 115.9, 66.9, 40.9; HRMS (ESI) Found: $[\mathrm{M}+\mathrm{H}]^{+}, 221.0810$. $\mathrm{C}_{12} \mathrm{H}_{12} \mathrm{O}_{4}$ requires $[\mathrm{M}+\mathrm{H}]^{+}, 221.0808$. 


\subsection{Anilide Formation from ß-Carbonyl Acids}

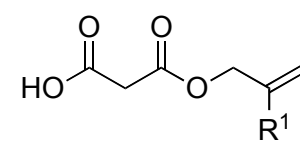

7

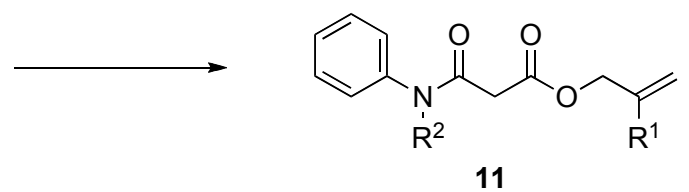

$\overbrace{1}^{O}$

12

Allyl 3-(methyl(phenyl)amino)-3-oxopropanoate (12). To a solution of malonic acid mono-ester 8 (5.22 g, $36.25 \mathrm{mmol})$ in dichloromethane $(100 \mathrm{~mL})$ was added 2-chloro-1methylpyridinium iodide $(13.90 \mathrm{~g}, 54.38 \mathrm{mmol})$, followed by $\mathrm{N}$-methylaniline $(4.31 \mathrm{~mL}$, $39.88 \mathrm{mmol})$. The mixture was cooled to $0^{\circ} \mathrm{C}$ and triethylamine $(25.2 \mathrm{~mL}, 181.3 \mathrm{mmol})$ was added dropwise. The resulting mixture was stirred at $0{ }^{\circ} \mathrm{C}$ for $5 \mathrm{~min}$, then allowed to warm to room temperature and stirred at this temperature for $30 \mathrm{~min}$. The reaction mixture was diluted with aq. $\mathrm{HCl}(10 \%, 100 \mathrm{~mL})$. The organic phase was separated, washed with aq. $\mathrm{NaHCO}_{3}(100 \mathrm{~mL})$ and brine $(100 \mathrm{~mL})$, then dried $\left(\mathrm{MgSO}_{4}\right)$ and concentrated in vacuo. Flash column chromatography [Petrol:EtOAc 2:1] afforded 12 (7.78 g, 92\%) as a pale yellow oil. $R_{F} 0.60$ [Petrol:EtOAc 1:1]; $v_{\max }(\mathrm{film}) / \mathrm{cm}^{-1} 3063,2943,1742,1664,1595 ; \delta_{H}$ (400 MHz, $\left.\mathrm{CDCl}_{3}\right)$ 7.45-7.39 (m, 2H), 7.38-7.33 (m, 1H), 7.25-7.21 (m, 2H), 5.87 (ddt, $J=$ 17.0, 10.5, $5.8 \mathrm{~Hz}, 1 \mathrm{H}$ ), 5.29 (dq, $J=17.2,1.5 \mathrm{~Hz}, 1 \mathrm{H}$ ), $5.22(\mathrm{dq}, J=10.4,1.2 \mathrm{~Hz}, 1 \mathrm{H}$ ), $4.55(\mathrm{td}, J=5.8,1.3 \mathrm{~Hz}, 2 \mathrm{H}), 3.31(\mathrm{~s}, 3 \mathrm{H}), 3.24(\mathrm{~s}, 2 \mathrm{H})$; $\delta_{\mathrm{C}}\left(100 \mathrm{MHz}, \mathrm{CDCl}_{3}\right) 167.3$, $165.8,143.4,131.6,129.9,128.3,127.2,118.6,65.8,41.4,37.4$; HRMS (ESI) Found: $[\mathrm{M}+\mathrm{H}]^{+}$, 234.1132. $\mathrm{C}_{13} \mathrm{H}_{15} \mathrm{NO}_{3}$ requires $[\mathrm{M}+\mathrm{H}]^{+}, 234.1125$.

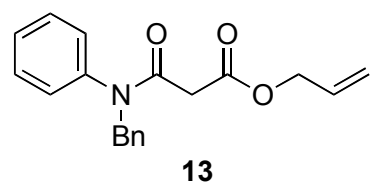

Allyl 3-(benzyl(phenyl)amino)-3-oxopropanoate (13). To a solution of malonic acid mono-ester 8 (1.44 g, $10 \mathrm{mmol})$ in dichloromethane $(40 \mathrm{~mL})$ was added 2-chloro-1methylpyridinium iodide $(3.83 \mathrm{~g}, 15 \mathrm{mmol})$, followed by $\mathrm{N}$-benzylaniline $(2.01 \mathrm{~g}, 11 \mathrm{mmol})$. The mixture was cooled to $0{ }^{\circ} \mathrm{C}$ and triethylamine $(7.0 \mathrm{~mL}, 50 \mathrm{mmol})$ was added dropwise. The resulting mixture was stirred at $0^{\circ} \mathrm{C}$ for $5 \mathrm{~min}$, then allowed to warm to room temperature and stirred at this temperature for $1 \mathrm{~h}$. The reaction mixture was diluted with 
aq. $\mathrm{HCl}(10 \%, 50 \mathrm{~mL})$. The organic phase was separated, washed with aq. $\mathrm{NaHCO}_{3}(50$ $\mathrm{mL})$ and brine $(100 \mathrm{~mL})$, then dried $\left(\mathrm{MgSO}_{4}\right)$ and concentrated in vacuo. Flash column chromatography [Petrol:EtOAc 6:1-4:1] afforded $13(2.96 \mathrm{~g}, 96 \%)$ as a pale yellow oil. $R_{F}$ 0.38 [Petrol:EtOAc 2:1]; $\mathrm{v}_{\max }(\mathrm{film}) / \mathrm{cm}^{-1} 3031,1739,1656,1595 ; \delta_{\mathrm{H}}\left(400 \mathrm{MHz}, \mathrm{CDCl}_{3}\right)$ 7.33-7.19 (m, 8H), 7.00 (dd, $J=6.8,3.0 \mathrm{~Hz}, 2 \mathrm{H}), 5.86(\mathrm{ddt}, J=17.2,10.4,5.8 \mathrm{~Hz}, 1 \mathrm{H})$, $5.29(\mathrm{dq}, J=17.2,1.5 \mathrm{~Hz}, 1 \mathrm{H}), 5.22(\mathrm{dq}, J=10.4,1.2 \mathrm{~Hz}, 1 \mathrm{H}), 4.91(\mathrm{~s}, 2 \mathrm{H}), 4.57$ (dt, $J=$ 5.8, $1.4 \mathrm{~Hz}, 2 \mathrm{H}), 3.24(\mathrm{~s}, 2 \mathrm{H})$; $\delta_{\mathrm{C}}\left(100 \mathrm{MHz} \mathrm{CDCl}_{3}\right)$ 167.3, 165.8, 141.6, 136.8, 131.6, 129.6, 128.7, 128.4, 128.3, 128.3, 127.4, 118.6, 65.8, 53.0, 41.7; HRMS (ESI) Found: $[\mathrm{M}+\mathrm{Na}]^{+}, 332.1257 . \mathrm{C}_{19} \mathrm{H}_{19} \mathrm{NO}_{3}$ requires $[\mathrm{M}+\mathrm{Na}]^{+}, 332.1257$.

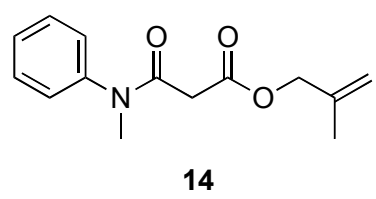

2-Methylallyl 3-(methyl(phenyl)amino)-3-oxopropanoate (14). To a solution of malonic acid mono-ester 9 (926 mg, $5.86 \mathrm{mmol})$ in dichloromethane $(20 \mathrm{~mL})$ was added 2-chloro1-methylpyridinium iodide $(2.24 \mathrm{~g}, 8.79 \mathrm{mmol})$, followed by $N$-methylaniline (697 $\mu \mathrm{L}, 6.45$ $\mathrm{mmol})$. The mixture was cooled to $0^{\circ} \mathrm{C}$ and triethylamine $(4.08 \mathrm{~mL}, 29.3 \mathrm{mmol})$ was added dropwise. The resulting mixture was stirred at $0{ }^{\circ} \mathrm{C}$ for $5 \mathrm{~min}$, then allowed to warm to room temperature and stirred at this temperature for $15 \mathrm{~h}$. The reaction mixture was diluted with aq. $\mathrm{HCl}(10 \%, 30 \mathrm{~mL})$. The organic phase was separated, washed with aq. $\mathrm{NaHCO}_{3}(30$ $\mathrm{mL})$ and brine $(30 \mathrm{~mL})$, then dried $\left(\mathrm{MgSO}_{4}\right)$ and concentrated in vacuo. Flash column chromatography [Petrol:EtOAc 3:1] afforded $14(1.38 \mathrm{~g}, 95 \%)$ as a pale yellow oil. $R_{F} 0.47$ [Petrol:EtOAc 1:1]; $v_{\max }\left(\right.$ film) $/ \mathrm{cm}^{-1} 2937,1739,1659,1595 ; \delta_{\mathrm{H}}\left(400 \mathrm{MHz}, \mathrm{CDCl}_{3}\right) 7.41$ (t, J $=7.5 \mathrm{~Hz}, 2 \mathrm{H}), 7.35(\mathrm{t}, J=7.3 \mathrm{~Hz}, 1 \mathrm{H}), 7.22(\mathrm{~d}, J=7.3 \mathrm{~Hz}, 2 \mathrm{H}), 4.93(\mathrm{~s}, 1 \mathrm{H}), 4.90(\mathrm{~s}, 1 \mathrm{H})$, 4.48 (s, 2H), 3.29 (s, 3H), 3.24 (s, 2H), 1.70 (s, 3H); $\delta_{\mathrm{C}}\left(100 \mathrm{MHz}, \mathrm{CDCl}_{3}\right)$ 167.4, 165.8, 143.4, 139.4, 129.9, 128.2, 127.2, 113.3, 68.3, 41.3, 37.4, 19.4; HRMS (ESI) Found: $[\mathrm{M}+\mathrm{Na}]^{+}, 270.1102 . \mathrm{C}_{14} \mathrm{H}_{17} \mathrm{NO}_{3}$ requires $[\mathrm{M}+\mathrm{Na}]^{+}, 270.1101$.

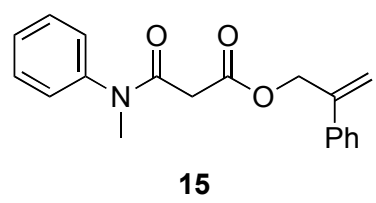

2-Phenylallyl 3-(methyl(phenyl)amino)-3-oxopropanoate (15). To a solution of malonic acid mono-ester 10 (1.165 g, $5.30 \mathrm{mmol})$ in dichloromethane (20 mL) was added 2-chloro1-methylpyridinium iodide $(2.03 \mathrm{~g}, 7.95 \mathrm{mmol})$, followed by $N$-methylaniline $(631 \mu \mathrm{L}, 5.83$ 
mmol). The mixture was cooled to $0^{\circ} \mathrm{C}$ and triethylamine $(3.69 \mathrm{~mL}, 26.5 \mathrm{mmol})$ was added dropwise. The resulting mixture was stirred at $0{ }^{\circ} \mathrm{C}$ for $5 \mathrm{~min}$, then allowed to warm to room temperature and stirred at this temperature for $1 \mathrm{~h}$. The reaction mixture was diluted with aq. $\mathrm{HCl}(10 \%, 30 \mathrm{~mL})$. The organic phase was separated, washed with aq. $\mathrm{NaHCO}_{3}(30$ $\mathrm{mL})$ and brine $(30 \mathrm{~mL})$, then dried $\left(\mathrm{MgSO}_{4}\right)$ and concentrated in vacuo. Flash column chromatography [Petrol:EtOAc 2:1] afforded $15(1.45 \mathrm{~g}, 89 \%)$ as a pale yellow oil. $R_{F} 0.19$ [Petrol:EtOAc 2:1]; $v_{\max }(\mathrm{film}) / \mathrm{cm}^{-1} 2937,1739,1656,1595 ; \delta_{\mathrm{H}}\left(400 \mathrm{MHz}, \mathrm{CDCl}_{3}\right) 7.40-$ $7.25(\mathrm{~m}, 8 \mathrm{H}), 7.11(\mathrm{dd}, J=8.0,1.6 \mathrm{~Hz}, 2 \mathrm{H}), 5.53(\mathrm{~s}, 1 \mathrm{H}), 5.33(\mathrm{~d}, J=0.9 \mathrm{~Hz}, 1 \mathrm{H}), 4.96(\mathrm{~d}$, $J=0.6 \mathrm{~Hz}, 2 \mathrm{H}), 3.25(\mathrm{~s}, 3 \mathrm{H}), 3.21(\mathrm{~s}, 2 \mathrm{H}) ; \delta_{\mathrm{C}}\left(100 \mathrm{MHz}, \mathrm{CDCl}_{3}\right)$ 167.3, 165.6, 143.3, $141.8,137.7,129.8,128.4,128.1,128.0,127.1,125.9,115.6,66.3,41.2$, 37.3; HRMS (ESI) Found: $[\mathrm{M}+\mathrm{Na}]^{+}, 332.1258 . \mathrm{C}_{19} \mathrm{H}_{19} \mathrm{NO}_{3}$ requires $[\mathrm{M}+\mathrm{Na}]^{+}, 332.1257$. 


\subsection{Dicarbonyl Alkylation}
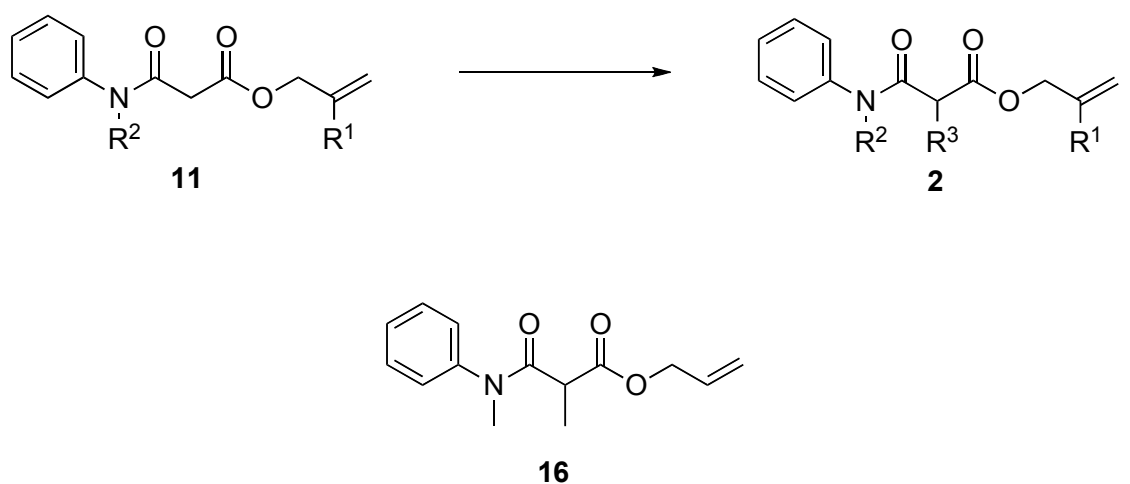

Allyl 2-methyl-3-(methyl(phenyl)amino)-3-oxopropanoate (16). To a solution of dicarbonyl $12(1.165 \mathrm{~g}, 5.0 \mathrm{mmol})$ in tetrahydrofuran $(15 \mathrm{~mL})$ was added potassium tertbutoxide $(616 \mathrm{mg}, 5.5 \mathrm{mmol}$ ) in one portion and the mixture was stirred for $5 \mathrm{~min}$. Methyl iodide (327 $\mu \mathrm{L}, 5.25 \mathrm{mmol}$ ) was added dropwise and the mixture was stirred at room temperature for $1 \mathrm{~h}$. The reaction mixture was diluted with aq. $\mathrm{HCl}(10 \%, 50 \mathrm{~mL})$ and extracted with EtOAc $(3 \times 50 \mathrm{~mL})$. The combined organic fractions were washed with brine $(100 \mathrm{~mL})$, dried $\left(\mathrm{MgSO}_{4}\right)$ and concentrated in vacuo. Flash column chromatography [Petrol:EtOAc 3:1] afforded 16 (946 mg, 77\%) as a clear oil. $R_{F} 0.54$ [Petrol:EtOAc 1:1]; $\mathrm{V}_{\max }(\mathrm{film}) / \mathrm{cm}^{-1} 2987,2941,1744,1661,1595 ; \delta_{\mathrm{H}}\left(400 \mathrm{MHz}, \mathrm{CDCl}_{3}\right)$ 7.46-7.40 (m, 2H), 7.39-7.33 (m, 1H), 7.26-7.22 (m, 2H), 5.86 (ddt, $J=17.1,10.5,1.5 \mathrm{~Hz}, 1 \mathrm{H}), 5.28$ (dq, $J=$ 17.2, $1.5 \mathrm{~Hz}, 1 \mathrm{H}$ ), $5.22(\mathrm{dq}, J=10.5,1.3 \mathrm{~Hz}, 1 \mathrm{H}), 4.61-4.49(\mathrm{~m}, 2 \mathrm{H}), 3.44(\mathrm{q}, J=7.1 \mathrm{~Hz}$, $1 \mathrm{H}), 3.30(\mathrm{~s}, 3 \mathrm{H}), 1.31(\mathrm{~d}, J=7.1 \mathrm{~Hz}, 3 \mathrm{H}) ; \delta_{\mathrm{C}}\left(100 \mathrm{MHz}, \mathrm{CDCl}_{3}\right) 170.1,169.7,143.3$, 131.6, 129.7, 128.0, 127.2, 117.9, 65.4, 43.2, 37.4, 13.9; HRMS (ESI) Found: $[\mathrm{M}+\mathrm{H}]^{+}$, 248.1281. $\mathrm{C}_{14} \mathrm{H}_{17} \mathrm{NO}_{3}$ requires $[\mathrm{M}+\mathrm{H}]^{+}, 248.1281$.

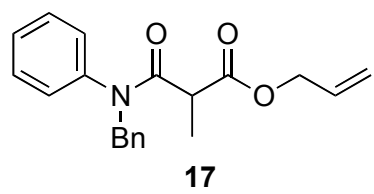

Allyl 3-(benzyl(phenyl)amino)-2-methyl-3-oxopropanoate (17). To a solution of dicarbonyl $13(618 \mathrm{mg}, 2.0 \mathrm{mmol})$ in tetrahydrofuran $(10 \mathrm{~mL})$ was added potassium tertbutoxide $(247 \mathrm{mg}, 2.2 \mathrm{mmol}$ ) in one portion and the mixture was stirred for $5 \mathrm{~min}$. Methyl iodide $(131 \mu \mathrm{L}, 2.1 \mathrm{mmol})$ was added dropwise and the mixture was stirred at room temperature for $2 \mathrm{~h}$. The reaction mixture was diluted with aq. $\mathrm{HCl}(10 \%, 50 \mathrm{~mL})$ and extracted with EtOAc $(3 \times 50 \mathrm{~mL})$. The combined organic fractions were washed with brine $(100 \mathrm{~mL})$, dried $\left(\mathrm{MgSO}_{4}\right)$ and concentrated in vacuo. Flash column chromatography 
[Petrol:EtOAc 6:1] afforded 17 (538 mg, 83\%) as a yellow oil. $R_{F} 0.53$ [Petrol:EtOAc 2:1]; $v_{\max }(\mathrm{film}) / \mathrm{cm}^{-1} 2937,1740,1655,1595 ; \delta_{\mathrm{H}}\left(400 \mathrm{MHz}, \mathrm{CDCl}_{3}\right)$ 7.32-7.28 (m, 3H), 7.26$7.18(\mathrm{~m}, 5 \mathrm{H}), 7.03-6.98(\mathrm{~m}, 2 \mathrm{H}), 5.85$ (ddt, $J=17.1,10.5,5.7 \mathrm{~Hz}, 1 \mathrm{H}$ ), 5.27 (dq, $J=17.2$, $1.5 \mathrm{~Hz}, 1 \mathrm{H}$ ), $5.21(\mathrm{dq}, J=10.5,1.2 \mathrm{~Hz}, 1 \mathrm{H}), 5.07(\mathrm{~d}, J=14.3 \mathrm{~Hz}, 1 \mathrm{H}), 4.72(\mathrm{~d}, J=14.3 \mathrm{~Hz}$, $1 \mathrm{H}$ ), 4.54 (ddd, $J=5.8,2.5,1.2 \mathrm{~Hz}, 2 \mathrm{H}$ ), 3.39 (q, $J=7.0 \mathrm{~Hz}, 1 \mathrm{H}$ ), $1.34(\mathrm{~d}, J=7.0 \mathrm{~Hz}, 3 \mathrm{H}$ ); $\delta_{\mathrm{C}}\left(100 \mathrm{MHz}, \mathrm{CDCl}_{3}\right)$ 170.3, 170.0, 141.7, 137.1, 131.7, 129.6, 128.7, 128.5, 128.3, 128.3, 127.3, 118.3, 65.7, 53.1, 43.7, 14.1; HRMS (ESI) Found: $[\mathrm{M}+\mathrm{Na}]^{+}$, 346.1413. $\mathrm{C}_{20} \mathrm{H}_{21} \mathrm{NO}_{3}$ requires $[\mathrm{M}+\mathrm{Na}]^{+}, 346.1414$.

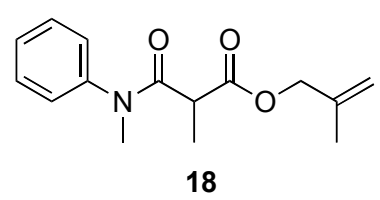

2-Methylallyl 2-methyl-3-(methyl(phenyl)amino)-3-oxopropanoate (18). To a solution of dicarbonyl $14(494 \mathrm{mg}, 2.0 \mathrm{mmol})$ in tetrahydrofuran $(10 \mathrm{~mL})$ was added potassium tertbutoxide $(247 \mathrm{mg}, 2.2 \mathrm{mmol}$ ) in one portion and the mixture was stirred for $5 \mathrm{~min}$. Methyl iodide (131 $\mu \mathrm{L}, 2.1 \mathrm{mmol}$ ) was added dropwise and the mixture was stirred at room temperature for $2 \mathrm{~h}$. The reaction mixture was diluted with aq. $\mathrm{HCl}(10 \%, 50 \mathrm{~mL})$ and extracted with EtOAc $(3 \times 50 \mathrm{~mL})$. The combined organic fractions were washed with brine $(100 \mathrm{~mL})$, dried $\left(\mathrm{MgSO}_{4}\right)$ and concentrated in vacuo. Flash column chromatography [Petrol:EtOAc 6:1-4:1] afforded 18 (420 mg, 80\%) as a yellow oil. $R_{F} 0.58$ [Petrol:EtOAc 2:1]; $v_{\max }(\mathrm{film}) / \mathrm{cm}^{-1} 2938,1741,1656,1595 ; \delta_{\mathrm{H}}\left(400 \mathrm{MHz}, \mathrm{CDCl}_{3}\right) 7.42$ (t, $J=7.4 \mathrm{~Hz}$, 2H), $7.36(\mathrm{t}, J=7.3 \mathrm{~Hz}, 1 \mathrm{H}), 7.26-7.22(\mathrm{~m}, 2 \mathrm{H}), 4.92(\mathrm{~d}, J=9.7 \mathrm{~Hz}, 2 \mathrm{H}), 4.51-4.42(\mathrm{~m}$, 2H), 3.45 (q, $J=7.0 \mathrm{~Hz}, 1 \mathrm{H}$ ), 3.29 (s, 3H), $1.71(\mathrm{~s}, 3 \mathrm{H}), 1.31$ (d, $J=7.0 \mathrm{~Hz}, 3 \mathrm{H}) ; \delta_{\mathrm{C}}(100$ $\left.\mathrm{MHz}, \mathrm{CDCl}_{3}\right)$ 170.3, 170.0, 143.6, 139.5, 129.9, 128.2, 127.4, 112.9, 68.1, 43.4, 37.6, 19.4, 14.1; HRMS (ESI) Found: $[\mathrm{M}+\mathrm{Na}]^{+}, 284.1258 . \mathrm{C}_{15} \mathrm{H}_{19} \mathrm{NO}_{3}$ requires $[\mathrm{M}+\mathrm{Na}]^{+}$, 284.1257.

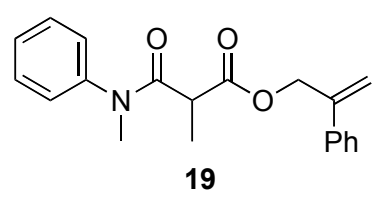

2-Phenylallyl 2-methyl-3-(methyl(phenyl)amino)-3-oxopropanoate (19). To a solution of dicarbonyl $15(927 \mathrm{mg}, 3.0 \mathrm{mmol})$ in tetrahydrofuran $(15 \mathrm{~mL})$ was added potassium tertbutoxide $(370 \mathrm{mg}, 3.3 \mathrm{mmol}$ ) in one portion and the mixture was stirred for $5 \mathrm{~min}$. Methyl iodide (196 $\mu \mathrm{L}, 3.15 \mathrm{mmol}$ ) was added dropwise and the mixture was stirred at room 
temperature for $1 \mathrm{~h}$. The reaction mixture was diluted with aq. $\mathrm{HCl}(10 \%, 50 \mathrm{~mL})$ and extracted with EtOAc $(3 \times 50 \mathrm{~mL})$. The combined organic fractions were washed with brine $(100 \mathrm{~mL})$, dried $\left(\mathrm{MgSO}_{4}\right)$ and concentrated in vacuo. Flash column chromatography [Petrol:EtOAc 4:1] afforded 19 (870 mg, 90\%) as a yellow oil. $R_{F} 0.35$ [Petrol:EtOAc 2:1]; $\mathrm{V}_{\max }(\mathrm{film}) / \mathrm{cm}^{-1} 2937,1742,1655,1595 ; \delta_{\mathrm{H}}\left(400 \mathrm{MHz}, \mathrm{CDCl}_{3}\right)$ 7.43-7.27 (m, 8H), 7.05$6.99(\mathrm{~m}, 2 \mathrm{H}), 5.55-5.53(\mathrm{~m}, 1 \mathrm{H}), 5.34-5.31(\mathrm{~m}, 1 \mathrm{H}), 5.00(\mathrm{dd}, J=13.2,0.8 \mathrm{~Hz}, 1 \mathrm{H}), 4.93$ (dd, $J=13.2,0.8 \mathrm{~Hz}, 1 \mathrm{H}$ ), 3.38 (q, $J=7.0 \mathrm{~Hz}, 1 \mathrm{H}), 3.20(\mathrm{~s}, 3 \mathrm{H}), 1.29(\mathrm{~d}, J=7.0 \mathrm{~Hz}, 3 \mathrm{H}$ ); $\delta_{\mathrm{C}}\left(100 \mathrm{MHz}, \mathrm{CDCl}_{3}\right)$ 170.2, 169.8, 143.4, 142.1, 137.9, 129.8, 128.4, 128.0, 128.0, 127.3, 126.0, 115.6, 66.3, 43.2, 37.4, 13.9; HRMS (ESI) Found: $[\mathrm{M}+\mathrm{H}]^{+}$, 324.1593. $\mathrm{C}_{20} \mathrm{H}_{21} \mathrm{NO}_{3}$ requires $[\mathrm{M}+\mathrm{H}]^{+}, 324.1594$.

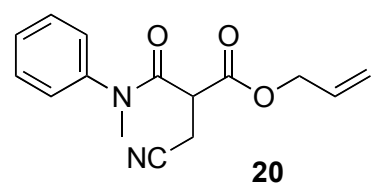

Allyl 2-(cyanomethyl)-3-(methyl(phenyl)amino)-3-oxopropanoate (20). To a solution of dicarbonyl 12 (932 mg, $4.0 \mathrm{mmol})$ in tetrahydrofuran $(20 \mathrm{~mL})$ was added potassium tertbutoxide (493 mg, $4.4 \mathrm{mmol}$ ) in one portion and the mixture was stirred for $5 \mathrm{~min}$. Bromoacetonitrile (293 $\mu \mathrm{L}, 4.2 \mathrm{mmol})$ was added dropwise and the mixture was stirred at room temperature for $2 \mathrm{~h}$. The reaction mixture was diluted with aq. $\mathrm{HCl}(10 \%, 50 \mathrm{~mL})$ and extracted with EtOAc $(3 \times 50 \mathrm{~mL})$. The combined organic fractions were washed with brine $(100 \mathrm{~mL})$, dried $\left(\mathrm{MgSO}_{4}\right)$ and concentrated in vacuo. Flash column chromatography [Petrol:EtOAc 3:1] afforded 20 (1.05 g, 97\%) as a pale pink solid. $R_{F} 0.26$ [Petrol:EtOAc 2:1]; mp 47-49 ${ }^{\circ} \mathrm{C} ; \mathrm{v}_{\max }(\mathrm{film}) / \mathrm{cm}^{-1} 2913,2158,1706,1610 ; \delta_{\mathrm{H}}\left(400 \mathrm{MHz}, \mathrm{CDCl}_{3}\right) 7.46(\mathrm{t}, \mathrm{J}$ $=7.4 \mathrm{~Hz}, 2 \mathrm{H}), 7.40(\mathrm{t}, J=7.4 \mathrm{~Hz}, 1 \mathrm{H}), 7.34-7.30(\mathrm{~m}, 2 \mathrm{H}), 5.85$ (ddt, $J=16.4,10.7,5.7 \mathrm{~Hz}$, 1H), 5.33-5.24 (m, 2H), 4.60 (ddt, $J=13.2,5.7,1.2 \mathrm{~Hz}, 1 \mathrm{H}), 4.56$ (ddt, $J=13.2,5.7,1.1$ $\mathrm{Hz}, 1 \mathrm{H}), 3.75$ (dd, J=9.6, $5.4 \mathrm{~Hz}, 1 \mathrm{H}), 3.36$ (s, 3H), 2.97 (dd, J = 16.9, $9.6 \mathrm{~Hz}, 1 \mathrm{H}), 2.79$ (dd, $J=16.9,5.4 \mathrm{~Hz}, 1 \mathrm{H}) ; \delta_{\mathrm{C}}\left(100 \mathrm{MHz}, \mathrm{CDCl}_{3}\right)$ 166.7, 165.9, 142.5, 130.9, 130.1, 128.7, 127.7, 119.1, 117.2, 66.6, 44.8, 37.9, 17.3; HRMS (ESI) Found: [M+Na $]^{+}, 295.1052$. $\mathrm{C}_{15} \mathrm{H}_{16} \mathrm{~N}_{2} \mathrm{O}_{3}$ requires $[\mathrm{M}+\mathrm{Na}]^{+}, 295.1053$.

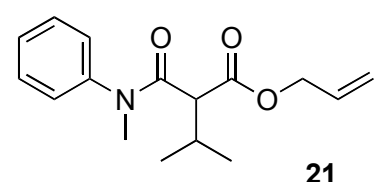


Allyl 3-methyl-2-(methyl(phenyl)carbamoyl)butanoate (21). A suspension of sodium hydride $(60 \%, 220 \mathrm{mg}, 5.5 \mathrm{mmol})$ in tetrahydrofuran $(15 \mathrm{~mL})$ was cooled to $0{ }^{\circ} \mathrm{C}$, and a solution of dicarbonyl $12(1.17 \mathrm{~g}, 5.0 \mathrm{mmol})$ in tetrahydrofuran $(5 \mathrm{~mL})$ was added dropwise via syringe. The solution was stirred at $0{ }^{\circ} \mathrm{C}$ for $15 \mathrm{~min}$, at which point 2-bromopropane $(0.94 \mathrm{~mL}, 10 \mathrm{mmol})$ and tetrabutylammonium iodide $(369 \mathrm{mg}, 1.0 \mathrm{mmol})$ were added. The mixture was stirred at $0{ }^{\circ} \mathrm{C}$ for a further $15 \mathrm{~min}$. A reflux condenser was fitted and the mixture was heated to $80{ }^{\circ} \mathrm{C}$ for 3 days. The mixture was allowed to cool to room temperature, diluted with aq. $\mathrm{HCl}(10 \%, 30 \mathrm{~mL})$ and extracted with EtOAc $(3 \times 50 \mathrm{~mL})$. The combined organic fractions were washed with brine $(150 \mathrm{~mL})$, dried $\left(\mathrm{MgSO}_{4}\right)$ and concentrated in vacuo. Flash column chromatography [Petrol:EtOAc 6:1] afforded 21 (763 $\mathrm{mg}, 56 \%)$ as a clear oil. $R_{F} 0.46$ [Petrol:EtOAc 2:1]; $\mathrm{v}_{\max }(\mathrm{film}) / \mathrm{cm}^{-1} 2964,1746,1655$, 1595; $\delta_{\mathrm{H}}\left(400 \mathrm{MHz}, \mathrm{CDCl}_{3}\right) 7.42(\mathrm{t}, J=7.3 \mathrm{~Hz}, 2 \mathrm{H}), 7.35(\mathrm{t}, J=7.3 \mathrm{~Hz}, 1 \mathrm{H}), 7.20(\mathrm{~d}, J=$ $7.0 \mathrm{~Hz}, 2 \mathrm{H}$ ), 5.87 (ddt, $J=17.2,10.5,5.7 \mathrm{~Hz}, 1 \mathrm{H}$ ), 5.29 (dq, $J=17.2,1.5 \mathrm{~Hz}, 1 \mathrm{H}$ ), 5.22 (dq, $J=10.4,1.3 \mathrm{~Hz}, 1 \mathrm{H}$ ), $4.56(\mathrm{dt}, J=5.7,1.4 \mathrm{~Hz}, 2 \mathrm{H}$ ), $3.29(\mathrm{~s}, 3 \mathrm{H}), 3.08(\mathrm{~d}, J=9.7 \mathrm{~Hz}$, $1 \mathrm{H})$, 2.53-2.39 (m, $1 \mathrm{H}), 0.90(\mathrm{~d}, J=6.7 \mathrm{~Hz}, 3 \mathrm{H}), 0.82(\mathrm{~d}, J=6.7 \mathrm{~Hz}, 3 \mathrm{H}) ; \delta_{\mathrm{C}}(100 \mathrm{MHz}$, $\left.\mathrm{CDCl}_{3}\right)$ 169.0, 168.1, 143.4, 131.8, 129.7, 128.1, 127.9, 118.4, 65.4, 56.3, 37.7, 29.6, 20.5, 20.5; HRMS (ESI) Found: $[\mathrm{M}+\mathrm{Na}]^{+}$, 298.1413. $\mathrm{C}_{16} \mathrm{H}_{21} \mathrm{NO}_{3}$ requires $[\mathrm{M}+\mathrm{Na}]^{+}, 298.1414$.

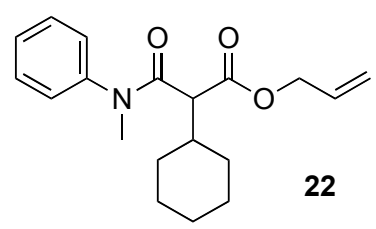

Allyl 2-methyl-3-(methyl(phenyl)amino)-3-oxopropanoate (22). A suspension of sodium hydride $(60 \%, 880 \mathrm{mg}, 22 \mathrm{mmol})$ in tetrahydrofuran $(40 \mathrm{~mL})$ was cooled to $0{ }^{\circ} \mathrm{C}$, and a solution of dicarbonyl $12(4.66 \mathrm{~g}, 20 \mathrm{mmol})$ in tetrahydrofuran $(10 \mathrm{~mL})$ was added dropwise via syringe. The solution was stirred at $0{ }^{\circ} \mathrm{C}$ for $15 \mathrm{~min}$, at which point iodocyclohexane $(5.17 \mathrm{~mL}, 40 \mathrm{mmol})$ was added. The mixture was stirred at $0{ }^{\circ} \mathrm{C}$ for a further $15 \mathrm{~min}$. A reflux condenser was fitted and the mixture was heated to $80^{\circ} \mathrm{C}$ for 4 days. The mixture was allowed to cool to room temperature, diluted with aq. $\mathrm{HCl}(10 \%, 50$ $\mathrm{mL})$ and extracted with EtOAc $(3 \times 100 \mathrm{~mL})$. The combined organic fractions were washed with brine $(200 \mathrm{~mL})$, dried $\left(\mathrm{MgSO}_{4}\right)$ and concentrated in vacuo. Flash column chromatography [Petrol:EtOAc 9:1] afforded $22(2.27 \mathrm{~g}, 36 \%)$ as a clear oil. $R_{F} 0.40$ [Petrol:EtOAc 2:1]; $v_{\max }(\mathrm{film}) / \mathrm{cm}^{-1} 2928,2851,1745,1656,1595 ; \delta_{\mathrm{H}}\left(400 \mathrm{MHz}, \mathrm{CDCl}_{3}\right)$ 7.43 (t, $J=7.4 \mathrm{~Hz}, 2 \mathrm{H}$ ), 7.36 (t, $J=7.3 \mathrm{~Hz}, 1 \mathrm{H}$ ), 7.19 (d, $J=7.0 \mathrm{~Hz}, 2 \mathrm{H}$ ), 5.88 (ddt, $J=$ 
17.1, 10.5, $5.7 \mathrm{~Hz}, 1 \mathrm{H}$ ), 5.30 (dq, $J=17.2,1.5 \mathrm{~Hz}, 1 \mathrm{H}$ ), $5.23(\mathrm{dq}, J=10.4,1.3 \mathrm{~Hz}, 1 \mathrm{H}$ ), 4.62-4.51 (m, 2H), $3.29(\mathrm{~s}, 3 \mathrm{H}), 3.12(\mathrm{~d}, J=10.0 \mathrm{~Hz}, 1 \mathrm{H}), 2.19$ (qt, $J=11.8,3.1 \mathrm{~Hz}, 1 \mathrm{H})$, $1.75(\mathrm{~d}, J=12.1 \mathrm{~Hz}, 1 \mathrm{H}), 1.66-1.56(\mathrm{~m}, 4 \mathrm{H}), 1.34-1.17(\mathrm{~m}, 2 \mathrm{H}), 1.11-0.97(\mathrm{~m}, 1 \mathrm{H}), 0.88-$ $0.66(\mathrm{~m}, 2 \mathrm{H}) ; \delta_{\mathrm{C}}\left(100 \mathrm{MHz}, \mathrm{CDCl}_{3}\right)$ 168.9, 167.9, 143.4, 131.8, 129.7, 128.1, 127.9, 118.4, 65.4, 55.5, 38.9, 37.7, 30.9, 30.8, 26.0, 26.0, 25.8; HRMS (ESI) Found: $[\mathrm{M}+\mathrm{H}]^{+}$, 316.1915. $\mathrm{C}_{19} \mathrm{H}_{25} \mathrm{NO}_{3}$ requires $[\mathrm{M}+\mathrm{H}]^{+}, 316.1907$. 


\subsection{Anilide Formation from Phenylacetic Acids}
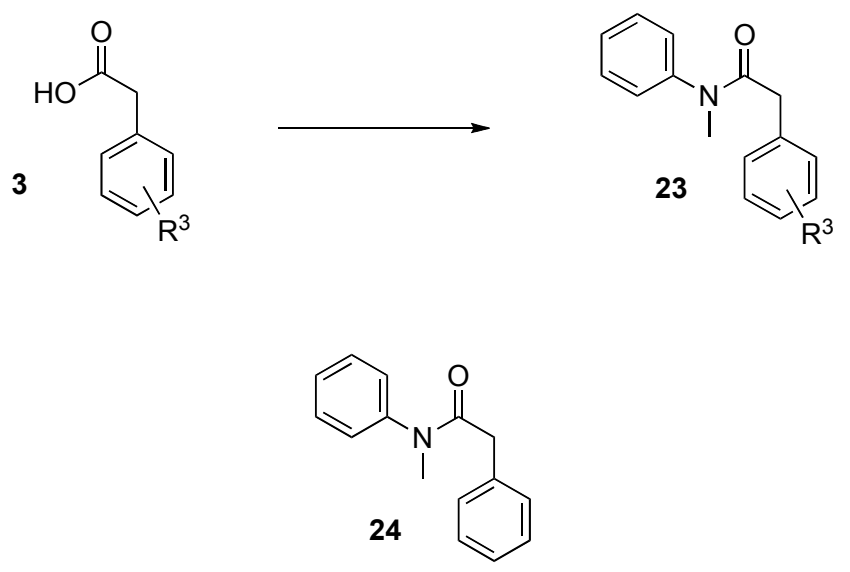

$\mathbf{N}$-Methyl-N,2-diphenylacetamide (24). To a solution of phenylacetic acid (4.08 g, 30 $\mathrm{mmol})$ in dichloromethane $(100 \mathrm{~mL})$ was added 2-chloro-1-methylpyridinium iodide (11.5 g, $45 \mathrm{mmol})$, followed by $N$-methylaniline $(3.57 \mathrm{~mL}, 33 \mathrm{mmol})$. The mixture was cooled to $0^{\circ} \mathrm{C}$ and triethylamine $(20.9 \mathrm{~mL}, 150 \mathrm{mmol})$ was added dropwise. The resulting mixture was allowed to warm to room temperature and stirred at this temperature for $1 \mathrm{~h}$. The reaction mixture was diluted with aq. $\mathrm{HCl}(10 \%, 100 \mathrm{~mL})$. The organic phase was separated, washed with aq. $\mathrm{NaHCO}_{3}(100 \mathrm{~mL})$ and brine $(100 \mathrm{~mL})$, then dried $\left(\mathrm{MgSO}_{4}\right)$ and concentrated in vacuo. Flash column chromatography [Petrol:EtOAc 3:1] afforded 24 (5.72 $\mathrm{g}, 85 \%)$ as a clear oil, identical to previously isolated material. ${ }^{3} R_{F} 0.53$ [Petrol:EtOAc 1:1]; $\mathrm{v}_{\max }(\mathrm{film}) / \mathrm{cm}^{-1} 3031,1652,1594 ; \delta_{\mathrm{H}}\left(400 \mathrm{MHz}, \mathrm{CDCl}_{3}\right)$ 7.43-7.32 (m, 3H), 7.26-7.15 (m, 3H), $7.12(\mathrm{~d}, J=7.1 \mathrm{~Hz}, 2 \mathrm{H}), 7.06(\mathrm{~d}, J=7.0 \mathrm{~Hz}, 2 \mathrm{H}), 3.46(\mathrm{~s}, 2 \mathrm{H}), 3.28$ $(\mathrm{s}, 3 \mathrm{H}) ; \delta_{\mathrm{C}}\left(100 \mathrm{MHz}, \mathrm{CDCl}_{3}\right)$ 170.8, 143.8, 135.3, 129.6, 128.9, 128.2, 127.8, 127.5, 126.4, 40.8, 37.4; HRMS (ESI) Found: $[\mathrm{M}+\mathrm{H}]^{+}$, 226.1229. $\mathrm{C}_{15} \mathrm{H}_{15} \mathrm{NO}$ requires $[\mathrm{M}+\mathrm{H}]^{+}$, 226.1226.

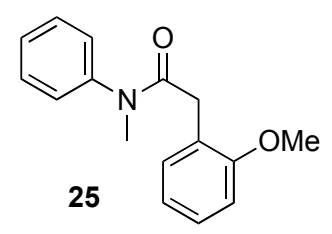

2-(2-Methoxyphenyl)- $N$-methyl- $N$-phenylacetamide (25). To a solution of 2methoxyphenylacetic acid (830 mg, $5.0 \mathrm{mmol}$ ) in dichloromethane $(20 \mathrm{~mL})$ was added 2chloro-1-methylpyridinium iodide $(1.91 \mathrm{~g}, 7.5 \mathrm{mmol})$, followed by $N$-methylaniline $(595 \mu \mathrm{L}$, $5.5 \mathrm{mmol})$. The mixture was cooled to $0^{\circ} \mathrm{C}$ and triethylamine $(3.48 \mathrm{~mL}, 25 \mathrm{mmol})$ was added dropwise. The resulting mixture was allowed to warm to room temperature and stirred at this temperature for $15 \mathrm{~h}$. The reaction mixture was diluted with aq. $\mathrm{HCl}(10 \%, 30$ 
$\mathrm{mL})$. The organic phase was separated, washed with aq. $\mathrm{NaHCO}_{3}(30 \mathrm{~mL})$ and brine $(30$ $\mathrm{mL})$, then dried $\left(\mathrm{MgSO}_{4}\right)$ and concentrated in vacuo. Flash column chromatography [Petrol:EtOAc 2:1] afforded 25 (1.17 g, 92\%) as a clear oil. $R_{F} 0.21$ [Petrol:EtOAc 2:1]; $\mathrm{V}_{\max }(\mathrm{film}) / \mathrm{cm}^{-1} 2937,1654,1595 ; \delta_{\mathrm{H}}\left(400 \mathrm{MHz}, \mathrm{CDCl}_{3}\right) 7.38(\mathrm{t}, J=7.5 \mathrm{~Hz}, 2 \mathrm{H}), 7.31$ (t, $J$ $=7.3 \mathrm{~Hz}, 1 \mathrm{H}), 7.22-7.14(\mathrm{~m}, 4 \mathrm{H}), 6.88(\mathrm{t}, J=7.4 \mathrm{~Hz}, 1 \mathrm{H}), 6.77(\mathrm{~d}, J=8.1 \mathrm{~Hz}, 1 \mathrm{H}), 3.69(\mathrm{~s}$, $3 \mathrm{H}), 3.45(\mathrm{~s}, 2 \mathrm{H}), 3.30(\mathrm{~s}, 3 \mathrm{H}) ; \delta_{\mathrm{C}}\left(100 \mathrm{MHz}, \mathrm{CDCl}_{3}\right)$ 171.2, 156.9, 144.1, 130.5, 129.3, 127.7, 127.4, 127.3, 124.3, 120.2, 110.0, 55.0, 37.4, 35.2; HRMS (ESI) Found: [M+Na] $]^{+}$, 278.1154. $\mathrm{C}_{16} \mathrm{H}_{17} \mathrm{NO}_{2}$ requires $[\mathrm{M}+\mathrm{Na}]^{+}, 278.1151$.

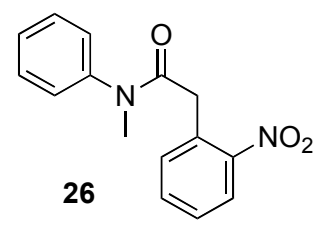

$\mathrm{N}$-Methyl-2-(2-nitrophenyl)-N-phenylacetamide (26). To a solution of 2-nitrophenylacetic acid (905 mg, $5.0 \mathrm{mmol}$ ) in dichloromethane (20 mL) was added 2-chloro-1methylpyridinium iodide $(1.91 \mathrm{~g}, 7.5 \mathrm{mmol})$, followed by $N$-methylaniline $(595 \mu \mathrm{L}, 5.5$ $\mathrm{mmol})$. The mixture was cooled to $0{ }^{\circ} \mathrm{C}$ and triethylamine $(3.48 \mathrm{~mL}, 25 \mathrm{mmol})$ was added dropwise. The resulting mixture was allowed to warm to room temperature and stirred at this temperature for $1 \mathrm{~h}$. The reaction mixture was diluted with aq. $\mathrm{HCl}(10 \%, 30 \mathrm{~mL})$. The organic phase was separated, washed with aq. $\mathrm{NaHCO}_{3}(30 \mathrm{~mL})$ and brine $(30 \mathrm{~mL})$, then dried $\left(\mathrm{MgSO}_{4}\right)$ and concentrated in vacuo. Flash column chromatography [Petrol:EtOAc 2:1] afforded $26(1.20 \mathrm{~g}, 89 \%)$ as a pale yellow solid, identical to previously isolated material. ${ }^{4} R_{F} 0.13$ [Petrol:EtOAc 2:1]; mp 83-85 ${ }^{\circ} \mathrm{C} ; v_{\max }\left(\right.$ film) $/ \mathrm{cm}^{-1} 2937,1732,1705$, $1654,1609,1518,1345 ; \delta_{\mathrm{H}}\left(400 \mathrm{MHz}, \mathrm{CDCl}_{3}\right) 8.06(\mathrm{dd}, J=8.2,1.2 \mathrm{~Hz}, 1 \mathrm{H}), 7.53(\mathrm{td}, J=$ 7.5, $1.3 \mathrm{~Hz}, 1 \mathrm{H}), 7.48$ (t, $J=7.5 \mathrm{~Hz}, 2 \mathrm{H}), 7.44-7.34(\mathrm{~m}, 4 \mathrm{H}), 7.25$ (d, $J=7.1 \mathrm{~Hz}, 1 \mathrm{H}), 3.76$ $(\mathrm{s}, 2 \mathrm{H}), 3.30(\mathrm{~s}, 3 \mathrm{H}) ; \delta_{\mathrm{C}}\left(100 \mathrm{MHz}, \mathrm{CDCl}_{3}\right)$ 168.9, 148.6, 143.5, 133.3, 133.1, 131.5, 129.8, 128.0, 127.8, 127.4, 124.8, 40.1, 37.2; HRMS (ESI) Found: $[\mathrm{M}+\mathrm{Na}]^{+}, 293.0896$. $\mathrm{C}_{15} \mathrm{H}_{14} \mathrm{~N}_{2} \mathrm{O}_{3}$ requires $[\mathrm{M}+\mathrm{Na}]^{+}, 293.0897$.

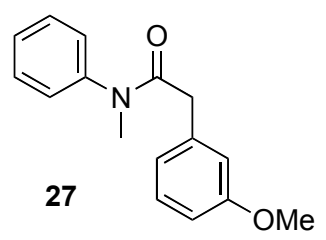

2-(3-Methoxyphenyl)- $N$-methyl- $N$-phenylacetamide (27). To a solution of 3methoxyphenylacetic acid $(830 \mathrm{mg}, 5.0 \mathrm{mmol})$ in dichloromethane $(20 \mathrm{~mL})$ was added 2- 
chloro-1-methylpyridinium iodide $(1.91 \mathrm{~g}, 7.5 \mathrm{mmol})$, followed by $\mathrm{N}$-methylaniline (595 $\mu \mathrm{L}$, $5.5 \mathrm{mmol})$. The mixture was cooled to $0{ }^{\circ} \mathrm{C}$ and triethylamine $(3.48 \mathrm{~mL}, 25 \mathrm{mmol})$ was added dropwise. The resulting mixture was allowed to warm to room temperature and stirred at this temperature for $1 \mathrm{~h}$. The reaction mixture was diluted with aq. $\mathrm{HCl}(10 \%, 30$ $\mathrm{mL})$. The organic phase was separated, washed with aq. $\mathrm{NaHCO}_{3}(30 \mathrm{~mL})$ and brine $(30$ $\mathrm{mL})$, then dried $\left(\mathrm{MgSO}_{4}\right)$ and concentrated in vacuo. Flash column chromatography [Petrol:EtOAc 2:1] afforded $27(1.15 \mathrm{~g}, 90 \%)$ as a clear oil. $R_{F} 0.16$ [Petrol:EtOAc 2:1]; $v_{\max }(\mathrm{film}) / \mathrm{cm}^{-1} 2929,1707,1653,1595 ; \delta_{\mathrm{H}}\left(400 \mathrm{MHz}, \mathrm{CDCl}_{3}\right) 7.39(\mathrm{t}, J=7.3 \mathrm{~Hz}, 2 \mathrm{H})$, 7.36-7.31 (m, 1H), 7.15-7.09 (m, 3H), $6.73(\mathrm{dd}, J=8.2,1.7 \mathrm{~Hz}, 1 \mathrm{H}), 6.64-6.59(\mathrm{~m}, 2 \mathrm{H})$, $3.73(\mathrm{~s}, 3 \mathrm{H}), 3.43(\mathrm{~s}, 2 \mathrm{H}), 3.27$ (s, 3H); $\delta_{\mathrm{C}}\left(100 \mathrm{MHz}, \mathrm{CDCl}_{3}\right)$ 170.7, 159.4, 143.8, 136.7, 129.5, 129.1, 127.8, 127.5, 121.3, 114.3, 112.2, 55.0, 40.8, 37.4; HRMS (ESI) Found: $[\mathrm{M}+\mathrm{H}]^{+}, 256.1323 . \mathrm{C}_{16} \mathrm{H}_{17} \mathrm{NO}_{2}$ requires $[\mathrm{M}+\mathrm{H}]^{+}, 256.1332$. 


\subsection{Allyloxycarbonylation of Phenylacetamides}
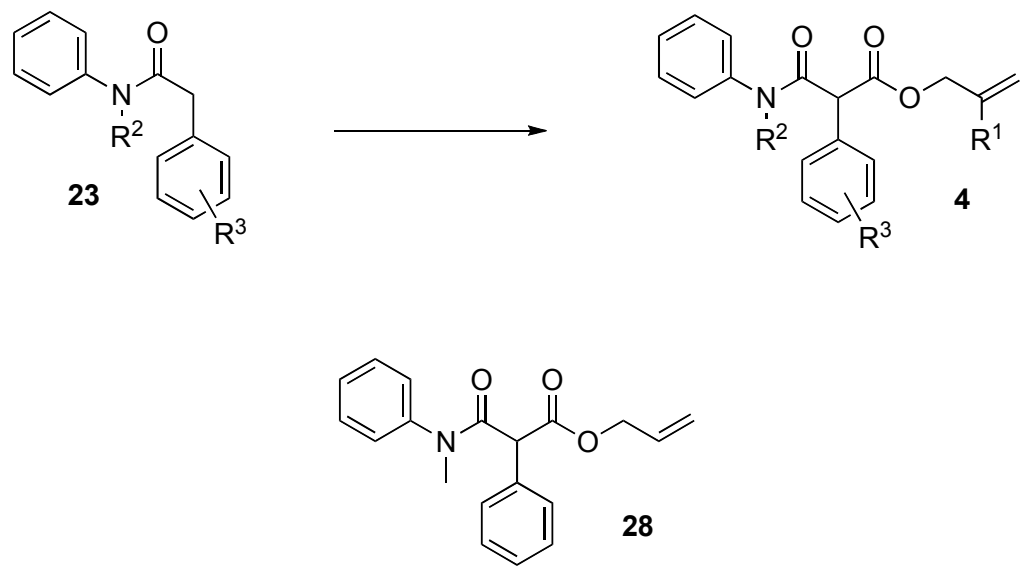

Allyl 3-(methyl(phenyl)amino)-3-oxo-2-phenylpropanoate (28). To a solution of 24 $(5.04 \mathrm{~g}, 22.4 \mathrm{mmol})$ in tetrahydrofuran $(20 \mathrm{~mL})$ cooled to $-78^{\circ} \mathrm{C}$ was added LiHMDS $(1 \mathrm{M}$ in THF, $47 \mathrm{~mL}, 47.0 \mathrm{mmol}$ ) dropwise and the resulting solution was stirred at $-78^{\circ} \mathrm{C}$ for 15 min. Allyl chloroformate $(2.38 \mathrm{~mL}, 22.4 \mathrm{mmol})$ was then added dropwise and the mixture was stirred at $-78{ }^{\circ} \mathrm{C}$ for $1 \mathrm{~h}$. The reaction was quenched with aq. $\mathrm{NH}_{4} \mathrm{Cl}(10 \mathrm{~mL})$ and allowed to warm to room temperature. The mixture was diluted with aq. $\mathrm{HCl}(10 \%, 50 \mathrm{~mL})$ and extracted with EtOAc $(3 \times 100 \mathrm{~mL})$. The combined organic fractions were washed with brine $(200 \mathrm{~mL})$, dried $\left(\mathrm{MgSO}_{4}\right)$ and concentrated in vacuo to afford crude 28 (6.90 g, quant.) as a clear oil, which was used in the next step without further purification. $R_{F} 0.44$ [Petrol:EtOAc 2:1]; $v_{\max }\left(\right.$ film) $/ \mathrm{cm}^{-1}$ 2937, 1748, 1656, 1595; $\delta_{\mathrm{H}}\left(400 \mathrm{MHz}, \mathrm{CDCl}_{3}\right) 7.42-$ $7.35(\mathrm{~m}, 3 \mathrm{H})$, 7.28-7.23 (m, 3H), 7.16-7.02 (m, 4H), 5.93-5.82 (m, 1H), $5.27(\mathrm{dq}, J=17.2$, $1.5 \mathrm{~Hz}, 1 \mathrm{H}), 5.20(\mathrm{dq}, J=10.5,1.3 \mathrm{~Hz}, 1 \mathrm{H}), 4.66-4.55(\mathrm{~m}, 3 \mathrm{H}), 3.27(\mathrm{~s}, 3 \mathrm{H}) ; \delta_{\mathrm{C}}(100 \mathrm{MHz}$, $\left.\mathrm{CDCl}_{3}\right)$ 168.4, 167.6, 143.1, 133.3, 131.7, 129.7, 129.4, 128.3, 128.2, 127.7, 127.7, 118.2, 65.9, 55.6, 37.7; HRMS (ESI) Found: $[\mathrm{M}+\mathrm{H}]^{+}$, 310.1445. $\mathrm{C}_{19} \mathrm{H}_{19} \mathrm{NO}_{3}$ requires $[\mathrm{M}+\mathrm{H}]^{+}$, 310.1438.

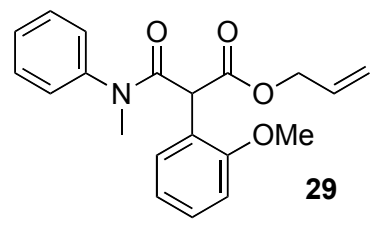

Allyl 2-(2-methoxyphenyl)-3-(methyl(phenyl)amino)-3-oxopropanoate (29). To a solution of 25 (993 mg, $3.89 \mathrm{mmol})$ in tetrahydrofuran $(10 \mathrm{~mL})$ cooled to $-78^{\circ} \mathrm{C}$ was added LiHMDS (1 M in THF, $8.18 \mathrm{~mL}, 8.18 \mathrm{mmol}$ ) dropwise and the resulting solution was stirred at $-78{ }^{\circ} \mathrm{C}$ for $15 \mathrm{~min}$. Allyl chloroformate $(414 \mu \mathrm{L}, 3.89 \mathrm{mmol})$ was then added dropwise 
and the mixture was stirred at $-78{ }^{\circ} \mathrm{C}$ for $30 \mathrm{~min}$. The reaction was quenched with aq. $\mathrm{NH}_{4} \mathrm{Cl}(5 \mathrm{~mL})$ and allowed to warm to room temperature. The mixture was diluted with aq. $\mathrm{HCl}(10 \%, 50 \mathrm{~mL})$ and extracted with EtOAc $(3 \times 100 \mathrm{~mL})$. The combined organic fractions were washed with brine $(200 \mathrm{~mL})$, dried $\left(\mathrm{MgSO}_{4}\right)$ and concentrated in vacuo to afford crude 29 (1.32 g, quant.) as a pale yellow oil, which was used in the next step without further purification. $R_{F} 0.30$ [Petrol:EtOAc 2:1]; $v_{\max }\left(\right.$ film) $/ \mathrm{cm}^{-1} 2937,1748,1655,1595 ; \delta_{\mathrm{H}}$ $\left(400 \mathrm{MHz}, \mathrm{CDCl}_{3}\right) 7.51(\mathrm{dd}, J=7.7,1.7 \mathrm{~Hz}, 1 \mathrm{H}), 7.33-7.28(\mathrm{~m}, 3 \mathrm{H}), 7.25-7.19(\mathrm{~m}, 1 \mathrm{H})$, 7.07-6.99 (m, 2H), 6.95 (td, J=7.5, $1.0 \mathrm{~Hz}, 1 \mathrm{H}), 6.67$ (dd, J = 8.3, 0.9 Hz, 1H), 5.93-5.82 (m, 1H), $5.26(\mathrm{dq}, J=17.2,1.6 \mathrm{~Hz}, 1 \mathrm{H}), 5.18(\mathrm{dq}, J=10.5,1.3 \mathrm{~Hz}, 1 \mathrm{H}), 5.14(\mathrm{~s}, 1 \mathrm{H}), 4.60$ (dt, $J=5.5,1.4 \mathrm{~Hz}, 2 \mathrm{H}), 3.46(\mathrm{~s}, 3 \mathrm{H}), 3.27$ (s, 3H); $\delta_{\mathrm{C}}\left(100 \mathrm{MHz}, \mathrm{CDCl}_{3}\right)$ 168.8, 168.3, 156.1, 143.1, 131.8, 130.0, 129.3, 128.8, 127.7, 127.6, 122.1, 120.4, 117.9, 109.8, 65.7, 54.8, 48.6, 37.6; HRMS (ESI) Found: $[\mathrm{M}+\mathrm{H}]^{+}$, 340.1538. $\mathrm{C}_{20} \mathrm{H}_{21} \mathrm{NO}_{4}$ requires $[\mathrm{M}+\mathrm{H}]^{+}$, 340.1543.

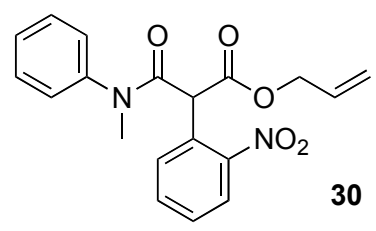

Allyl 3-(methyl(phenyl)amino)-2-(2-nitrophenyl)-3-oxopropanoate (30). To a solution of 26 (879 mg, $3.26 \mathrm{mmol})$ in tetrahydrofuran $(10 \mathrm{~mL})$ cooled to $-78{ }^{\circ} \mathrm{C}$ was added LiHMDS (1 M in THF, $6.84 \mathrm{~mL}, 6.84 \mathrm{mmol}$ ) dropwise and the resulting solution was stirred at $-78^{\circ} \mathrm{C}$ for $15 \mathrm{~min}$. Allyl chloroformate (346 $\mu \mathrm{L}, 3.26 \mathrm{mmol}$ ) was then added dropwise at $-78{ }^{\circ} \mathrm{C}$. The reaction was allowed to warm to room temperature and stirred at this temperature for $15 \mathrm{~h}$. The reaction was quenched with aq. $\mathrm{NH}_{4} \mathrm{Cl}(5 \mathrm{~mL})$, diluted with aq. $\mathrm{HCl}(10 \%, 50 \mathrm{~mL})$ and extracted with EtOAc $(3 \times 100 \mathrm{~mL})$. The combined organic fractions were washed with brine $(200 \mathrm{~mL})$, dried $\left(\mathrm{MgSO}_{4}\right)$ and concentrated in vacuo. Flash column chromatography [Petrol:EtOAc 3:1] afforded 30 (401 mg, 35\%) as a brown solid. $R_{F} 0.25$ [Petrol:EtOAc 2:1]; mp 86-88 ${ }^{\circ} \mathrm{C} ; \mathrm{v}_{\max }(\mathrm{film}) / \mathrm{cm}^{-1}$ 2937, 1732, 1706, 1655, 1524, 1346; $\delta_{\mathrm{H}}$ $\left(400 \mathrm{MHz} \mathrm{CDCl}_{3}\right) 7.86(\mathrm{dd}, J=8.2,1.3 \mathrm{~Hz}, 1 \mathrm{H}), 7.76(\mathrm{dd}, J=7.9,1.4 \mathrm{~Hz}, 1 \mathrm{H}), 7.65$ (td, J $=7.7,1.4 \mathrm{~Hz}, 1 \mathrm{H}), 7.48-7.42(\mathrm{~m}, 1 \mathrm{H}), 7.36-7.28(\mathrm{~m}, 3 \mathrm{H}), 7.07-6.91(\mathrm{~m}, 2 \mathrm{H}), 5.86(\mathrm{ddt}, J=$ 17.2, 10.5, $5.7 \mathrm{~Hz}, 1 \mathrm{H}), 5.31-5.25(\mathrm{~m}, 2 \mathrm{H}), 5.22(\mathrm{dq}, J=10.5,1.3 \mathrm{~Hz}, 1 \mathrm{H}), 4.66$ (ddt, $J=$ 13.2, 5.7, $1.4 \mathrm{~Hz}, 1 \mathrm{H}), 4.60$ (ddt, $J=13.2,5.6,1.4 \mathrm{~Hz}, 1 \mathrm{H}), 3.31(\mathrm{~s}, 3 \mathrm{H})$; $\delta_{\mathrm{C}}(100 \mathrm{MHz}$, $\left.\mathrm{CDCl}_{3}\right)$ 167.7, 166.8, 148.3, 142.3, 133.4, 131.5, 131.4, 130.0, 128.9, 128.8, 128.6, 127.4, 
124.7, 118.6, 66.4, 52.2, 37.8; $\mathrm{HRMS}$ (ESI) Found: $[\mathrm{M}+\mathrm{Na}]^{+}$, 377.1102. $\mathrm{C}_{19} \mathrm{H}_{18} \mathrm{~N}_{2} \mathrm{O}_{5}$ requires $[\mathrm{M}+\mathrm{Na}]^{+}, 377.1108$.

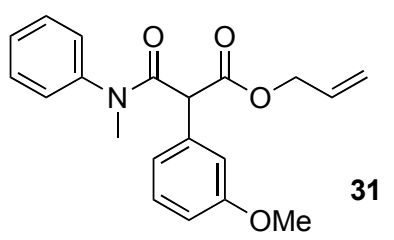

Allyl 2-(3-methoxyphenyl)-3-(methyl(phenyl)amino)-3-oxopropanoate (31). To a solution of 27 (979 mg, $3.84 \mathrm{mmol})$ in tetrahydrofuran $(10 \mathrm{~mL})$ cooled to $-78^{\circ} \mathrm{C}$ was added LiHMDS (1 M in THF, $8.06 \mathrm{~mL}, 8.06 \mathrm{mmol}$ ) dropwise and the resulting solution was stirred at $-78{ }^{\circ} \mathrm{C}$ for $15 \mathrm{~min}$. Allyl chloroformate $(408 \mu \mathrm{L}, 3.84 \mathrm{mmol})$ was then added dropwise and the mixture was stirred at $-78{ }^{\circ} \mathrm{C}$ for $30 \mathrm{~min}$. The reaction was quenched with aq. $\mathrm{NH}_{4} \mathrm{Cl}(5 \mathrm{~mL})$ and allowed to warm to room temperature. The mixture was diluted with aq. $\mathrm{HCl}(10 \%, 50 \mathrm{~mL})$ and extracted with EtOAc $(3 \times 100 \mathrm{~mL})$. The combined organic fractions were washed with brine $(200 \mathrm{~mL})$, dried $\left(\mathrm{MgSO}_{4}\right)$ and concentrated in vacuo to afford crude 31 (1.30 g, quant.) as a pale yellow oil, which was used in the next step without further purification. $R_{F} 0.33$ [Petrol:EtOAc 2:1]; $v_{\max }\left(\right.$ film) $/ \mathrm{cm}^{-1} 2937,1749,1656,1595 ; \delta_{H}$ (400 MHz, $\left.\mathrm{CDCl}_{3}\right)$ 7.43-7.34 (m, 3H), $7.16(\mathrm{t}, J=7.9 \mathrm{~Hz}, 1 \mathrm{H}), 7.10$ (br s, 2H), 6.81 (ddd, J = 8.3, 2.6, $0.9 \mathrm{~Hz}, 1 \mathrm{H}), 6.74-6.72(\mathrm{~m}, 1 \mathrm{H}), 6.68(\mathrm{dt}, J=7.6,1.0 \mathrm{~Hz}, 1 \mathrm{H}), 5.88(\mathrm{ddt}, J=$ 17.2, 10.6, $5.6 \mathrm{~Hz}, 1 \mathrm{H}), 5.28$ (dq, $J=17.2,1.6 \mathrm{~Hz}, 1 \mathrm{H}), 5.21(\mathrm{dq}, J=10.5,1.3 \mathrm{~Hz}, 1 \mathrm{H})$, 4.66-4.55 (m, 3H), $3.75(\mathrm{~s}, 3 \mathrm{H}), 3.28(\mathrm{~s}, 3 \mathrm{H}) ; \delta_{\mathrm{C}}\left(100 \mathrm{MHz}, \mathrm{CDCl}_{3}\right)$ 168.3, 167.4, 159.3, 143.0, 134.6, 131.6, 129.7, 129.0, 128.2, 127.7, 121.7, 118.1, 114.6, 113.7, 65.9, 55.5, 55.0, 37.6; HRMS (ESI) Found: $[\mathrm{M}+\mathrm{Na}]^{+}, 362.1351 . \mathrm{C}_{20} \mathrm{H}_{21} \mathrm{NO}_{4}$ requires $[\mathrm{M}+\mathrm{Na}]^{+}$, 362.1363. 


\subsection{Oxindole Cyclization}
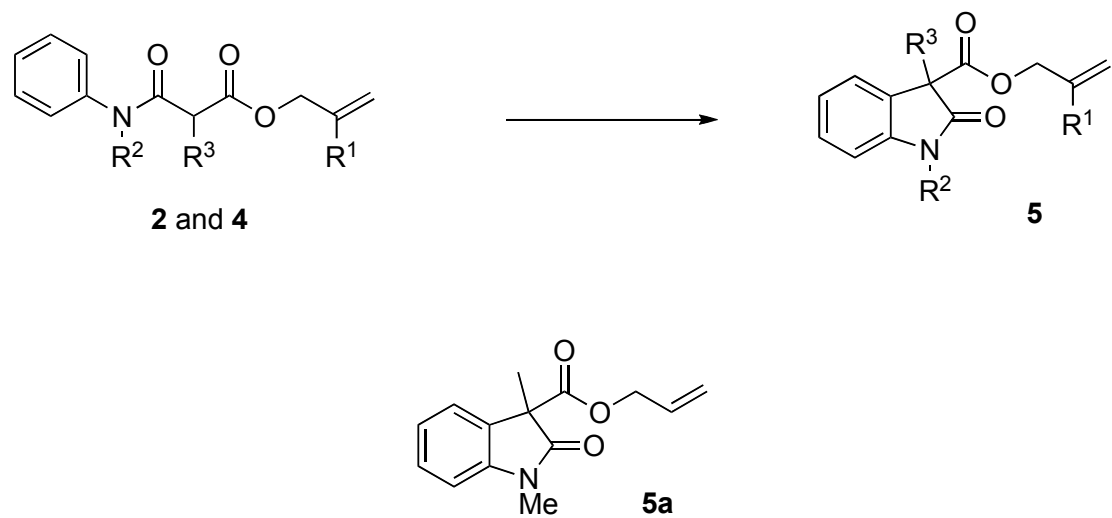

Allyl 1,3-dimethyl-2-oxoindoline-3-carboxylate (5a). To a solution of linear substrate 16 (933 $\mathrm{mg}, 3.77 \mathrm{mmol}$ ) in toluene $(60 \mathrm{~mL}$ ) was added copper(II) acetate monohydrate (752 $\mathrm{mg}, 3.77 \mathrm{mmol}$ ) and the resulting suspension was heated to $120{ }^{\circ} \mathrm{C}$ under an air atmosphere for $15 \mathrm{~h}$. The mixture was allowed to cool to room temperature, filtered through a plug of celite and concentrated in vacuo. Flash column chromatography [Petrol:EtOAc 9:1-6:1] afforded 5 a $(752 \mathrm{mg}, 81 \%)$ as a pale yellow solid. $R_{F} 0.67$ [Petrol:EtOAc 1:1]; mp 48-50 ${ }^{\circ} \mathrm{C} ; \mathrm{v}_{\max }(\mathrm{film}) / \mathrm{cm}^{-1}$ 2984, 2936, 1743, 1718, 1610; $\delta_{\mathrm{H}}(400$ $\left.\mathrm{MHz}, \mathrm{CDCl}_{3}\right) 7.33(\mathrm{td}, J=7.7,1.1 \mathrm{~Hz}, 1 \mathrm{H}), 7.28-7.24(\mathrm{~m}, 1 \mathrm{H}), 7.07(\mathrm{td}, J=7.5,0.7 \mathrm{~Hz}$, $1 \mathrm{H}), 6.87(\mathrm{~d}, J=7.8 \mathrm{~Hz}, 1 \mathrm{H}), 5.82-5.72(\mathrm{~m}, 1 \mathrm{H}), 5.16-5.09(\mathrm{~m}, 2 \mathrm{H}), 4.58-4.55(\mathrm{~m}, 2 \mathrm{H})$, $3.26(\mathrm{~s}, 3 \mathrm{H}), 1.68(\mathrm{~s}, 3 \mathrm{H}) ; \delta_{\mathrm{C}}\left(100 \mathrm{MHz}, \mathrm{CDCl}_{3}\right)$ 174.8, 169.1, 143.4, 131.1, 129.7, 128.8, 122.7, 122.6, 117.6, 108.3, 65.7, 54.8, 26.3, 19.8. HRMS (ESI) Found: $[\mathrm{M}+\mathrm{H}]^{+}, 246.1125$. $\mathrm{C}_{14} \mathrm{H}_{15} \mathrm{NO}_{3}$ requires $[\mathrm{M}+\mathrm{H}]^{+}, 246.1125$.

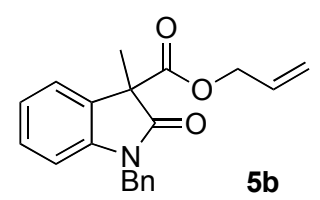

Allyl 1-benzyl-3-methyl-2-oxoindoline-3-carboxylate (5b). To a solution of linear substrate 17 (431 mg, $1.33 \mathrm{mmol})$ in toluene $(50 \mathrm{~mL})$ was added copper(II) acetate monohydrate $\left(266 \mathrm{mg}, 1.33 \mathrm{mmol}\right.$ ) and the resulting suspension was heated to $120^{\circ} \mathrm{C}$ under an air atmosphere for $15 \mathrm{~h}$. The mixture was allowed to cool to room temperature, filtered through a plug of celite and concentrated in vacuo. Flash column chromatography [Petrol:EtOAc 9:1] afforded $\mathbf{5 b}(377 \mathrm{mg}, 88 \%)$ as a brown solid. $R_{F} 0.30$ [Petrol:EtOAc 4:1]; mp 55-58 ${ }^{\circ} \mathrm{C} ; \mathrm{v}_{\max }(\mathrm{film}) / \mathrm{cm}^{-1} 2937,1737,1709,1611 ; \delta_{\mathrm{H}}\left(400 \mathrm{MHz}, \mathrm{CDCl}_{3}\right) 7.32-$ $7.21(\mathrm{~m}, 6 \mathrm{H}), 7.18(\mathrm{td}, J=7.8,1.3 \mathrm{~Hz}, 1 \mathrm{H}), 7.02(\mathrm{td}, J=7.6,1.0 \mathrm{~Hz}, 1 \mathrm{H}), 6.70(\mathrm{~d}, J=7.8$ $\mathrm{Hz}, 1 \mathrm{H}$ ), 5.78 (ddt, $J=17.5,10.3,5.5 \mathrm{~Hz}, 1 \mathrm{H}), 5.18-5.11(\mathrm{~m}, 3 \mathrm{H}), 4.75(\mathrm{~d}, J=15.8 \mathrm{~Hz}$, 
1H), 4.62 (ddt, $J=13.4,5.5,1.5 \mathrm{~Hz}, 1 \mathrm{H}$ ), 4.54 (ddt, $J=13.4,5.6,1.4 \mathrm{~Hz}, 1 \mathrm{H}), 1.73(\mathrm{~s}, 3 \mathrm{H}$ ); $\delta_{\mathrm{C}}\left(100 \mathrm{MHz}, \mathrm{CDCl}_{3}\right)$ 175.2, 169.3, 142.6, 135.4, 131.2, 130.0, 128.9, 128.7, 127.5, 127.0, 122.9, 122.8, 118.3, 109.5, 66.1, 55.0, 43.7, 19.8; HRMS (ESI) Found: [M+H] ${ }^{+}, 322.1443$. $\mathrm{C}_{20} \mathrm{H}_{19} \mathrm{NO}_{3}$ requires $[\mathrm{M}+\mathrm{H}]^{+}, 322.1438$.

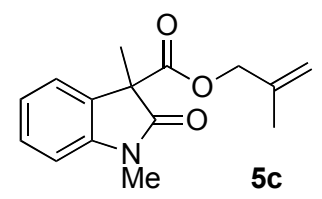

2-Methylallyl 1,3-dimethyl-2-oxoindoline-3-carboxylate (5c). To a solution of linear substrate 18 (355 mg, $1.36 \mathrm{mmol})$ in toluene $(40 \mathrm{~mL})$ was added copper(II) acetate monohydrate $(271 \mathrm{mg}, 1.36 \mathrm{mmol})$ and the resulting suspension was heated to $120{ }^{\circ} \mathrm{C}$ under an air atmosphere for $15 \mathrm{~h}$. The mixture was allowed to cool to room temperature, filtered through a plug of celite and concentrated in vacuo. Flash column chromatography [Petrol:EtOAc 6:1] afforded 5c (263 mg, 75\%) as a pale yellow oil. $R_{F} 0.43$ [Petrol:EtOAc 2:1]; $\mathrm{v}_{\max }(\mathrm{film}) / \mathrm{cm}^{-1} 2979,2937,1740,1714,1609 ; \delta_{\mathrm{H}}\left(400 \mathrm{MHz}, \mathrm{CDCl}_{3}\right) 7.32$ (td, J = 7.7, $1.2 \mathrm{~Hz}, 1 \mathrm{H}), 7.27-7.23(\mathrm{~m}, 1 \mathrm{H}), 7.06(\mathrm{td}, J=7.6,0.9 \mathrm{~Hz}, 1 \mathrm{H}), 6.87(\mathrm{~d}, J=7.8 \mathrm{~Hz}, 1 \mathrm{H})$, 4.81-4.78 (m, 1H), 4.75 (s, 1H), 4.49 (d, J = 13.4 Hz, 1H), 4.45 (d, J=13.4 Hz, 1H), 3.25 $(\mathrm{s}, 3 \mathrm{H}), 1.68(\mathrm{~s}, 3 \mathrm{H}), 1.57(\mathrm{~s}, 3 \mathrm{H}) ; \delta_{\mathrm{C}}\left(100 \mathrm{MHz}, \mathrm{CDCl}_{3}\right)$ 175.0, 169.3, 143.6, 139.2, 130.0, 129.0, 123.0, 122.8, 112.5, 108.4, 68.4, 55.0, 26.5, 19.8, 19.1; HRMS (ESI) Found: $[\mathrm{M}+\mathrm{H}]^{+}, 260.1278 . \mathrm{C}_{15} \mathrm{H}_{17} \mathrm{NO}_{3}$ requires $[\mathrm{M}+\mathrm{H}]^{+}, 260.1281$.

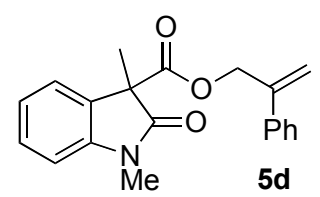

2-Phenylallyl 1,3-dimethyl-2-oxoindoline-3-carboxylate (5d). To a solution of linear substrate 19 (743 mg, $2.3 \mathrm{mmol})$ in toluene $(100 \mathrm{~mL})$ was added copper(II) acetate monohydrate $(458 \mathrm{mg}, 2.3 \mathrm{mmol})$ and the resulting suspension was heated to $120{ }^{\circ} \mathrm{C}$ under an air atmosphere for $24 \mathrm{~h}$. The mixture was allowed to cool to room temperature, filtered through a plug of celite and concentrated in vacuo. Flash column chromatography [Petrol:EtOAc 6:1] afforded 5d (586 mg, 79\%) as a yellow oil. $R_{F} 0.21$ [Petrol:EtOAc 4:1]; $\mathrm{V}_{\max }(\mathrm{film}) / \mathrm{cm}^{-1} 2979,2937,1740,1714,1609 ; \delta_{\mathrm{H}}\left(400 \mathrm{MHz}, \mathrm{CDCl}_{3}\right) 7.29$ (td, J = 7.7, 1.3 $\mathrm{Hz}, 1 \mathrm{H}), 7.27-7.20(\mathrm{~m}, 5 \mathrm{H}), 7.11(\mathrm{~d}, J=7.4 \mathrm{~Hz}, 1 \mathrm{H}), 7.00(\mathrm{td}, J=7.6,0.9 \mathrm{~Hz}, 1 \mathrm{H}), 6.81$ (d, $J=7.8 \mathrm{~Hz}, 1 \mathrm{H}), 5.39(\mathrm{~s}, 1 \mathrm{H}), 5.14-5.12(\mathrm{~m}, 1 \mathrm{H}), 4.98(\mathrm{~d}, J=13.5 \mathrm{~Hz}, 1 \mathrm{H}), 4.91(\mathrm{~d}, J=$ $13.5 \mathrm{~Hz}, 1 \mathrm{H}), 3.18$ (s, 3H), 1.66 (s, 3H); $\delta_{\mathrm{C}}\left(100 \mathrm{MHz}, \mathrm{CDCl}_{3}\right)$ 174.8, 169.2, 143.5, 141.9, 
137.7, 129.7, 128.9, 128.3, 127.8, 125.9, 122.9, 122.8, 114.8, 108.3, 66.6, 55.0, 26.4, 19.5; HRMS (ESI) Found: $[\mathrm{M}+\mathrm{H}]^{+}, 322.1432 . \mathrm{C}_{20} \mathrm{H}_{19} \mathrm{NO}_{3}$ requires $[\mathrm{M}+\mathrm{H}]^{+}, 322.1438$.

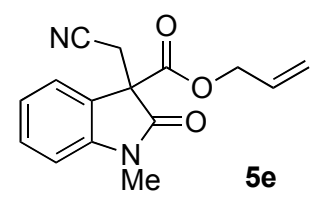

Allyl 3-(cyanomethyl)-1-methyl-2-oxoindoline-3-carboxylate (5e). To a solution of linear substrate 20 (920 mg, $3.38 \mathrm{mmol})$ in mesitylene (100 mL) was added copper(II) acetate monohydrate $(673 \mathrm{mg}, 3.38 \mathrm{mmol})$ and the resulting suspension was heated to $170{ }^{\circ} \mathrm{C}$ under an air atmosphere for $15 \mathrm{~h}$. The mixture was allowed to cool to room temperature, filtered through a plug of celite and concentrated in vacuo. Flash column chromatography [Petrol:EtOAc 3:1] afforded 5 e $(361 \mathrm{mg}, 40 \%)$ as a dark orange solid. $R_{F}$ 0.27 [Petrol:EtOAc 2:1]; mp 70-72 ${ }^{\circ} \mathrm{C} ; \mathrm{v}_{\max }(\mathrm{film}) / \mathrm{cm}^{-1} 2913,1706,1610 ; \delta_{\mathrm{H}}(400 \mathrm{MHz}$, $\left.\mathrm{CDCl}_{3}\right) 7.42(\mathrm{td}, J=7.8,1.2 \mathrm{~Hz}, 1 \mathrm{H}), 7.37(\mathrm{dd}, J=7.5,0.7 \mathrm{~Hz}, 1 \mathrm{H}), 7.14$ (td, $J=7.6,1.0$ $\mathrm{Hz}, 1 \mathrm{H}), 6.94(\mathrm{~d}, J=7.9 \mathrm{~Hz}, 1 \mathrm{H}), 5.74$ (ddt, $J=17.1,10.7,5.4 \mathrm{~Hz}, 1 \mathrm{H}), 5.17-5.08(\mathrm{~m}, 2 \mathrm{H})$, 4.59 (dt, $J=5.4,1.5 \mathrm{~Hz}, 2 \mathrm{H}), 3.34$ (d, $J=16.8 \mathrm{~Hz}, 1 \mathrm{H}), 3.28$ (s, 3H), 3.04 (d, $J=16.8 \mathrm{~Hz}$, $1 \mathrm{H}) ; \delta_{\mathrm{C}}\left(100 \mathrm{MHz}, \mathrm{CDCl}_{3}\right)$ 171.3, 166.7, 144.1, 130.6, 130.5, 125.3, 123.5, 123.4, 118.6, 115.5, 109.2, 66.8, 55.5, 26.8, 22.5; HRMS (ESI) Found: $[\mathrm{M}+\mathrm{H}]^{+}, 271.1077 . \mathrm{C}_{15} \mathrm{H}_{14} \mathrm{~N}_{2} \mathrm{O}_{3}$ requires $[\mathrm{M}+\mathrm{H}]^{+}, 271.1077$.

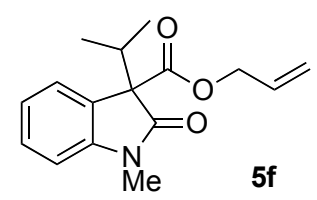

Allyl 3-isopropyl-1-methyl-2-oxoindoline-3-carboxylate (5f). To a solution of linear substrate 21 (654 mg, $2.38 \mathrm{mmol})$ in toluene $(50 \mathrm{~mL}$ ) was added copper(II) acetate monohydrate $\left(473 \mathrm{mg}, 2.38 \mathrm{mmol}\right.$ ) and the resulting suspension was heated to $120{ }^{\circ} \mathrm{C}$ under an air atmosphere for 4 days. The mixture was allowed to cool to room temperature, filtered through a plug of celite and concentrated in vacuo. Flash column chromatography [Petrol:EtOAc 6:1] afforded $\mathbf{5 f}(381 \mathrm{mg}, 59 \%)$ as a yellow oil. $R_{F} 0.26$ [Petrol:EtOAc 4:1]; $\mathrm{V}_{\max }(\mathrm{film}) / \mathrm{cm}^{-1} 2965,1740,1713,1609 ; \delta_{\mathrm{H}}\left(400 \mathrm{MHz}, \mathrm{CDCl}_{3}\right)$ 7.35-7.28 (m, 2H), 7.07 (td, $J=7.6,1.0 \mathrm{~Hz}, 1 \mathrm{H}), 6.83(\mathrm{~d}, J=7.7 \mathrm{~Hz}, 1 \mathrm{H}), 5.83(\mathrm{ddt}, J=17.2,10.6,5.5 \mathrm{~Hz}, 1 \mathrm{H}), 5.21$ (dq, $J=17.2,1.6 \mathrm{~Hz}, 1 \mathrm{H}), 5.16(\mathrm{dq}, J=10.5,1.3 \mathrm{~Hz}, 1 \mathrm{H}), 4.61$ (dt, $J=5.5,1.5 \mathrm{~Hz}, 2 \mathrm{H}$ ), $3.22(\mathrm{~s}, 3 \mathrm{H}), 2.85-2.73(\mathrm{~m}, 1 \mathrm{H}), 1.04(\mathrm{~d}, J=6.8 \mathrm{~Hz}, 3 \mathrm{H}), 0.81(\mathrm{~d}, J=6.9 \mathrm{~Hz}, 3 \mathrm{H}) ; \delta_{\mathrm{C}}(100$ $\left.\mathrm{MHz}_{\mathrm{CDCl}}\right)$ 173.0, 168.8, 144.0, 131.4, 128.8, 127.3, 124.2, 122.6, 118.2, 107.9, 65.9, 
63.3, 34.8, 26.2, 17.1, 17.1; HRMS (ESI) Found: $[\mathrm{M}+\mathrm{Na}]^{+}$, 296.1255. $\mathrm{C}_{16} \mathrm{H}_{19} \mathrm{NO}_{3}$ requires $[\mathrm{M}+\mathrm{Na}]^{+}, 296.1257$.

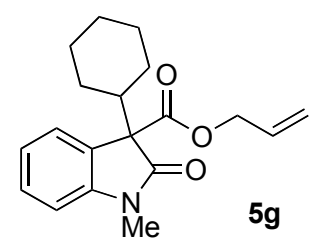

Allyl 3-cyclohexyl-1-methyl-2-oxoindoline-3-carboxylate $(\mathbf{5 g})$. To a solution of linear substrate $22(2.27 \mathrm{~g}, 7.21 \mathrm{mmol})$ in mesitylene $(100 \mathrm{~mL})$ was added copper(II) acetate monohydrate $(1.58 \mathrm{~g}, 7.93 \mathrm{mmol})$ and the resulting suspension was heated to $170{ }^{\circ} \mathrm{C}$ under an air atmosphere for $15 \mathrm{~h}$. The mixture was allowed to cool to room temperature, filtered through a plug of celite and concentrated in vacuo. Flash column chromatography [Petrol:EtOAc 14:1] afforded $5 \mathbf{g}(1.07 \mathrm{~g}, 47 \%)$ as a pale brown solid. $R_{F} 0.40$ [Petrol:EtOAc 2:1]; mp 72-74 ${ }^{\circ} \mathrm{C} ; \mathrm{v}_{\max }(\mathrm{film}) / \mathrm{cm}^{-1} 2937,2860,1715,1609 ; \delta_{\mathrm{H}}\left(400 \mathrm{MHz}, \mathrm{CDCl}_{3}\right) 7.35$ (dd, $J=7.5,1.1 \mathrm{~Hz}, 1 \mathrm{H}), 7.31(\mathrm{td}, J=7.7,1.2 \mathrm{~Hz}, 1 \mathrm{H}), 7.08(\mathrm{td}, J=7.6,1.0 \mathrm{~Hz}, 1 \mathrm{H}), 6.82(\mathrm{~d}, J$ $=7.8 \mathrm{~Hz}, 1 \mathrm{H}), 5.83(\mathrm{ddt}, J=17.2,10.8,5.5 \mathrm{~Hz}, 1 \mathrm{H}), 5.22(\mathrm{dq}, J=17.2,1.6 \mathrm{~Hz}, 1 \mathrm{H}), 5.17$ (dq, $J=10.5,1.3 \mathrm{~Hz}, 1 \mathrm{H}), 4.61$ (dt, $J=5.4,1.5 \mathrm{~Hz}, 2 \mathrm{H}$ ), 3.22 (s, 3H), 2.45 (tt, J=12.0, 3.0 $\mathrm{Hz}, 1 \mathrm{H}), 1.76-1.57(\mathrm{~m}, 4 \mathrm{H}), 1.48-0.95(\mathrm{~m}, 6 \mathrm{H}) ; \delta_{\mathrm{C}}\left(100 \mathrm{MHz}, \mathrm{CDCl}_{3}\right)$ 172.9, 168.5, 143.8, 131.3, 128.6, 127.2, 124.2, 122.5, 118.1, 107.8, 65.8, 63.3, 44.8, 26.9, 26.8, 26.2, 26.2, 26.1, 25.8; HRMS (ESI) Found: $[\mathrm{M}+\mathrm{H}]^{+}, 314.1754 . \mathrm{C}_{19} \mathrm{H}_{23} \mathrm{NO}_{3}$ requires $[\mathrm{M}+\mathrm{H}]^{+}, 314.1751$.

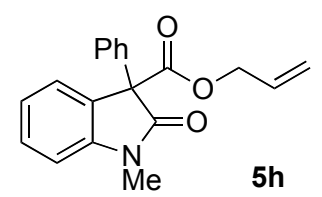

Allyl 1-methyl-2-oxo-3-phenylindoline-3-carboxylate (5h). To a solution of linear substrate 28 (577 mg, $1.87 \mathrm{mmol})$ in toluene $(30 \mathrm{~mL})$ was added copper(II) acetate monohydrate $(37 \mathrm{mg}, 0.19 \mathrm{mmol})$ and the resulting suspension was heated to $120{ }^{\circ} \mathrm{C}$ under an air atmosphere for $15 \mathrm{~h}$. The mixture was allowed to cool to room temperature, filtered through a plug of celite and concentrated in vacuo. Flash column chromatography [Petrol:EtOAc 4:1] afforded $5 \mathrm{~h}(471 \mathrm{mg}, 82 \%)$ as a pale yellow solid. $R_{F} 0.15$ [Petrol:EtOAc 4:1]; mp 89-91 ${ }^{\circ} \mathrm{C} ; \mathrm{v}_{\max }\left(\right.$ film) $/ \mathrm{cm}^{-1}$ 2945, 1737, 1704, 1607; $\delta_{\mathrm{H}}(400 \mathrm{MHz}$, $\left.\mathrm{CDCl}_{3}\right) 7.47(\mathrm{dd}, J=7.5,0.8 \mathrm{~Hz}, 1 \mathrm{H}), 7.42(\mathrm{td}, J=7.8,1.3 \mathrm{~Hz}, 1 \mathrm{H}), 7.37-7.30(\mathrm{~m}, 5 \mathrm{H})$, 7.17 (td, $J=7.6,1.0 \mathrm{~Hz}, 1 \mathrm{H}), 6.93(\mathrm{~d}, J=7.8 \mathrm{~Hz}, 1 \mathrm{H}), 5.81$ (ddt, $J=17.2,10.7,5.5 \mathrm{~Hz}$, $1 \mathrm{H}), 5.18(\mathrm{dq}, J=17.2,1.6 \mathrm{~Hz}, 1 \mathrm{H}), 5.16(\mathrm{dq}, J=10.4,1.3 \mathrm{~Hz}, 1 \mathrm{H}), 4.68(\mathrm{ddt}, J=13.4$, 
5.4, $1.5 \mathrm{~Hz}, 1 \mathrm{H}), 4.63$ (ddt, $J=13.4,5.5,1.6 \mathrm{~Hz}, 1 \mathrm{H}), 3.23(\mathrm{~s}, 3 \mathrm{H}) ; \delta_{\mathrm{C}}\left(100 \mathrm{MHz}, \mathrm{CDCl}_{3}\right)$ 172.5, 168.7, 144.2, 135.6, 131.1, 129.6, 128.4, 128.1, 127.8, 126.7, 125.9, 122.8, 118.3, 108.6, 66.3, 63.8, 26.6; HRMS (ESI) Found: $[\mathrm{M}+\mathrm{H}]^{+}, 308.1285 . \mathrm{C}_{19} \mathrm{H}_{17} \mathrm{NO}_{3}$ requires $[\mathrm{M}+\mathrm{H}]^{+}, 308.1281$.

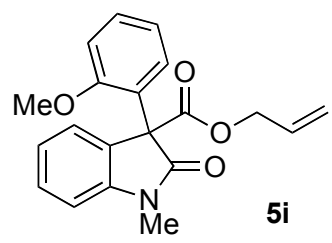

Allyl 3-(2-methoxyphenyl)-1-methyl-2-oxoindoline-3-carboxylate (5i). To a solution of linear substrate 29 (1.14 g, $3.35 \mathrm{mmol})$ in toluene $(100 \mathrm{~mL})$ was added copper(II) acetate monohydrate $\left(667 \mathrm{mg}, 3.35 \mathrm{mmol}\right.$ ) and the resulting suspension was heated to $120{ }^{\circ} \mathrm{C}$ under an air atmosphere for $48 \mathrm{~h}$. The mixture was allowed to cool to room temperature, filtered through a plug of celite and concentrated in vacuo. Flash column chromatography [Petrol:EtOAc 3:1-2:1] afforded $5 \mathbf{i}$ (850 mg, 75\%) as a yellow crystalline solid. $R_{F} 0.20$ [Petrol:EtOAc 2:1]; mp 83-86 ${ }^{\circ} \mathrm{C} ; \mathrm{v}_{\max }(\mathrm{film}) / \mathrm{cm}^{-1}$ 2937, 1732, 1706, 1608; $\delta_{\mathrm{H}}(400 \mathrm{MHz}$, $\left.\mathrm{CDCl}_{3}\right) 7.43(\mathrm{dd}, J=7.5,0.8 \mathrm{~Hz}, 1 \mathrm{H}), 7.34(\mathrm{td}, J=7.8,1.3 \mathrm{~Hz}, 1 \mathrm{H}), 7.26(\mathrm{ddd}, J=8.2,7.1$, $2.3 \mathrm{~Hz}, 1 \mathrm{H}), 7.06(\mathrm{td}, J=7.6,1.0 \mathrm{~Hz}, 1 \mathrm{H}), 7.00(\mathrm{dd}, J=8.2,1.0 \mathrm{~Hz}, 1 \mathrm{H}), 6.89-6.80(\mathrm{~m}$, $3 \mathrm{H}$ ), 5.86 (ddt, $J=17.2,10.5,5.6 \mathrm{~Hz}, 1 \mathrm{H}), 5.22(\mathrm{dq}, J=17.2,1.6 \mathrm{~Hz}, 1 \mathrm{H}), 5.16(\mathrm{dq}, J=$ 10.5, $1.3 \mathrm{~Hz}, 1 \mathrm{H}$ ), 4.68 (ddt, $J=13.3,5.6,1.4 \mathrm{~Hz}, 1 \mathrm{H}$ ), 4.62 (ddt, $J=13.3,5.6,1.4 \mathrm{~Hz}$, $1 \mathrm{H}), 3.84(\mathrm{~s}, 3 \mathrm{H}), 3.24(\mathrm{~s}, 3 \mathrm{H}) ; \delta_{\mathrm{C}}\left(100 \mathrm{MHz}, \mathrm{CDCl}_{3}\right)$ 172.4, 168.6, 157.9, 143.9, 131.7, 131.7, 129.5, 129.2, 128.1, 126.4, 125.8, 122.9, 120.8, 118.2, 112.6, 108.2, 66.3, 62.4, 56.1, 26.6; HRMS (ESI) Found: $[\mathrm{M}+\mathrm{H}]^{+}, 338.1383 . \mathrm{C}_{20} \mathrm{H}_{19} \mathrm{NO}_{4}$ requires $[\mathrm{M}+\mathrm{H}]^{+}, 338.1387$.

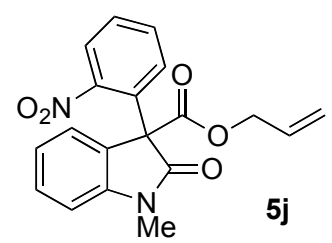

Allyl 1-methyl-3-(2-nitrophenyl)-2-oxoindoline-3-carboxylate (5j). To a solution of linear substrate 30 (329 mg, $0.93 \mathrm{mmol})$ in toluene $(50 \mathrm{~mL})$ was added copper(II) acetate monohydrate $(185 \mathrm{mg}, 0.93 \mathrm{mmol})$ and the resulting suspension was heated to $120{ }^{\circ} \mathrm{C}$ under an air atmosphere for $15 \mathrm{~h}$. The mixture was allowed to cool to room temperature, filtered through a plug of celite and concentrated in vacuo. Flash column chromatography [Petrol:EtOAc 3:1] afforded 5j (230 mg, 70\%) as a sticky brown oil. $R_{F} 0.19$ [Petrol:EtOAc 2:1]; $v_{\max }\left(\right.$ film)/cm ${ }^{-1} 2937,1735,1707,1608,1525,1347 ; \delta_{\mathrm{H}}\left(400 \mathrm{MHz}, \mathrm{CDCl}_{3}\right)$ 8.01-7.97 
(m, 1H), 7.54-7.45 (m, 3H), $7.42(\mathrm{td}, J=7.8,1.3 \mathrm{~Hz}, 1 \mathrm{H}), 7.30-7.26(\mathrm{~m}, 1 \mathrm{H}), 7.13(\mathrm{td}, J=$ 7.6, $1.0 \mathrm{~Hz}, 1 \mathrm{H}), 6.94(\mathrm{~d}, J=7.8 \mathrm{~Hz}, 1 \mathrm{H}), 5.87-5.76(\mathrm{~m}, 1 \mathrm{H}), 5.17-5.11(\mathrm{~m}, 2 \mathrm{H}), 4.64(\mathrm{dt}, J$ $=5.7,1.4 \mathrm{~Hz}, 2 \mathrm{H}), 3.28(\mathrm{~s}, 3 \mathrm{H}) ; \delta_{\mathrm{C}}\left(100 \mathrm{MHz}, \mathrm{CDCl}_{3}\right)$ 171.5, 167.2, 149.5, 144.1, 132.9, $131.1,130.8,130.1,130.1$, 129.3, 126.4, 126.2, 125.9, 123.2, 118.7, 108.9, 66.8, 63.5, 27.0; HRMS (ESI) Found: $[\mathrm{M}+\mathrm{H}]^{+}$, 353.1132. $\mathrm{C}_{19} \mathrm{H}_{16} \mathrm{~N}_{2} \mathrm{O}_{5}$ requires $[\mathrm{M}+\mathrm{H}]^{+}, 353.1132$.

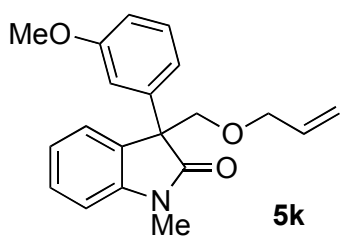

Allyl 3-(3-methoxyphenyl)-1-methyl-2-oxoindoline-3-carboxylate (5k). To a solution of linear substrate 31 (1.05 g, $3.1 \mathrm{mmol})$ in toluene $(100 \mathrm{~mL})$ was added copper(II) acetate monohydrate $(617 \mathrm{mg}, 3.1 \mathrm{mmol})$ and the resulting suspension was heated to $120{ }^{\circ} \mathrm{C}$ under an air atmosphere for $15 \mathrm{~h}$. The mixture was allowed to cool to room temperature, filtered through a plug of celite and concentrated in vacuo. Flash column chromatography [Petrol:EtOAc 4:1] afforded 5k (925 mg, 89\%) as a brown oil. $R_{F} 0.13$ [Petrol:EtOAc 4:1]; $\mathrm{V}_{\max }(\mathrm{film}) / \mathrm{cm}^{-1} 2937,1741,1714,1607,1583 ; \delta_{\mathrm{H}}\left(400 \mathrm{MHz}, \mathrm{CDCl}_{3}\right) 7.47$ (ddd, J = 7.5, 1.3, $0.5 \mathrm{~Hz}, 1 \mathrm{H}), 7.41(\mathrm{td}, J=7.8,1.3 \mathrm{~Hz}, 1 \mathrm{H}), 7.24(\mathrm{t}, J=8.0 \mathrm{~Hz}, 1 \mathrm{H}), 7.16(\mathrm{td}, J=7.6$, $1.0 \mathrm{~Hz}, 1 \mathrm{H}), 6.96-6.90(\mathrm{~m}, 3 \mathrm{H}), 6.85$ (ddd, $J=8.3,2.6,0.9 \mathrm{~Hz}, 1 \mathrm{H}), 5.87-5.76(\mathrm{~m}, 1 \mathrm{H})$, 5.22-5.14 (m, 2H), 4.68 (ddt, $J=13.4,5.4,1.5 \mathrm{~Hz}, 1 \mathrm{H}), 4.63$ (ddt, $J=13.4,5.5,1.5 \mathrm{~Hz}$, $1 \mathrm{H}), 3.76(\mathrm{~s}, 3 \mathrm{H}), 3.22(\mathrm{~s}, 3 \mathrm{H}) ; \delta_{\mathrm{C}}\left(100 \mathrm{MHz}, \mathrm{CDCl}_{3}\right)$ 172.4, 168.5, 159.4, 144.2, 137.0, 131.1, 129.6, 129.3, 126.6, 125.9, 122.8, 120.2, 118.4, 113.9, 113.3, 108.6, 66.3, 63.7, 55.1, 26.6; HRMS (ESI) Found: $[\mathrm{M}+\mathrm{H}]^{+}, 338.1384 . \mathrm{C}_{20} \mathrm{H}_{19} \mathrm{NO}_{4}$ requires $[\mathrm{M}+\mathrm{H}]^{+}, 338.1387$. 


\subsection{Decarboxylative Allyl Transfer}
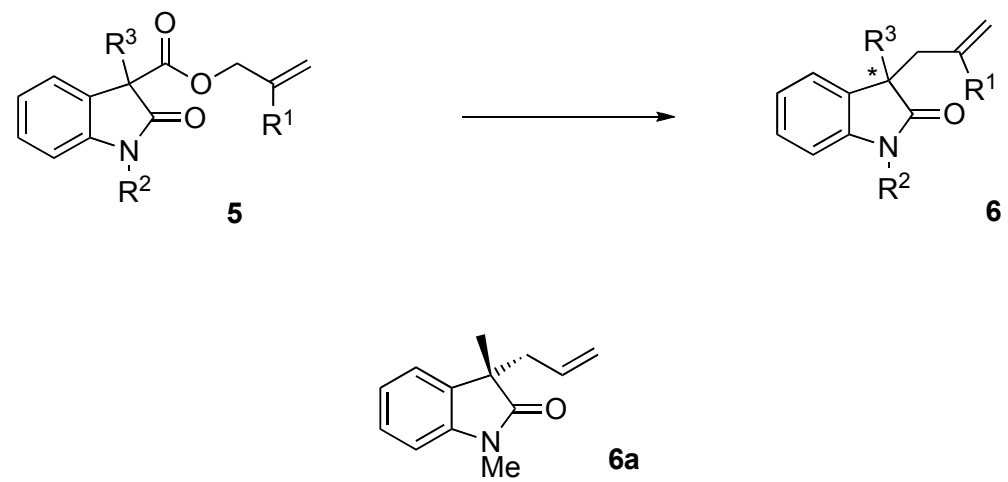

(S)-3-Allyl-1,3-dimethylindolin-2-one (6a). To a mixture of substrate 5a (37 mg, 0.15 $\mathrm{mmol}$ ), tris(dibenzylideneacetone)dipalladium(0) $(3.4 \mathrm{mg}, 3.75 \mu \mathrm{mol})$ and $(R, R)$-ANDENPhenyl-Trost ligand L6 $(7.9 \mathrm{mg}, 9.75 \mu \mathrm{mol})$ under an argon atmosphere was added dry dimethoxyethane $(3 \mathrm{~mL})$ and the mixture immediately cooled to $-25^{\circ} \mathrm{C}$ with stirring. The mixture was stirred at this temperature for $24 \mathrm{~h}$, then concentrated in vacuo. Flash column chromatography [Petrol:EtOAc 9:1] afforded $\mathbf{6 a}(25 \mathrm{mg}, 83 \%)$ as a pale yellow oil, identical to previously isolated material. ${ }^{5} R_{F} 0.59$ [Petrol:EtOAc 4:1]; $v_{\max }\left(\right.$ film) $/ \mathrm{cm}^{-1} 2928,2158$, 1705,$1611 ; \delta_{\mathrm{H}}\left(400 \mathrm{MHz}, \mathrm{CDCl}_{3}\right) 7.26(\mathrm{td}, J=7.7,1.3 \mathrm{~Hz}, 1 \mathrm{H}), 7.19(\mathrm{dd}, J=7.3,0.8 \mathrm{~Hz}$, $1 \mathrm{H}), 7.06(\mathrm{td}, J=7.5,1.0 \mathrm{~Hz}, 1 \mathrm{H}), 6.83(\mathrm{~d}, J=7.8 \mathrm{~Hz}, 1 \mathrm{H}$ ), 5.44 (ddt, $J=17.2,10.1,7.3$ $\mathrm{Hz}, 1 \mathrm{H}$ ), 4.98 (ddt, $J=17.0,2.0,1.4 \mathrm{~Hz}, 1 \mathrm{H}$ ), 4.92 (ddt, $J=10.1,1.8,0.7 \mathrm{~Hz}, 1 \mathrm{H}$ ), 3.20 (s, 3H), 2.53 (ddt, $J=13.5,7.6,1.0 \mathrm{~Hz}, 1 \mathrm{H}$ ), 2.50 (ddt, $J=13.5,7.1,1.1 \mathrm{~Hz}, 1 \mathrm{H}$ ), $1.37(\mathrm{~s}, 3 \mathrm{H}$ ); $\delta_{\mathrm{C}}\left(100 \mathrm{MHz}, \mathrm{CDCl}_{3}\right) 180.1,143.1,133.5,132.5,127.7,122.8,122.3,118.6,107.8,48.2$, 42.4, 26.0, 22.6; HRMS (ESI) Found: $[\mathrm{M}+\mathrm{H}]^{+}, 202.1227 . \mathrm{C}_{13} \mathrm{H}_{15} \mathrm{NO}$ requires $[\mathrm{M}+\mathrm{H}]^{+}$, 202.1226; Chiral HPLC, AD column, $1 \mathrm{~mL} / \mathrm{min}$, 99:1 Hexanes:IPA, $t_{A}$ (major) $=10.1 \mathrm{~min}, t_{B}$ $($ minor $)=12.1 \mathrm{~min}, 74 \% \mathrm{ee} ;[\mathrm{a}]_{D}^{25}-6.2\left(c 0.62, \mathrm{CHCl}_{3}, 74 \%\right.$ ee $)$.

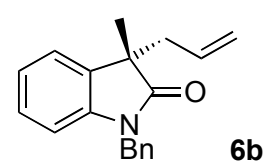

(S)-3-Allyl-1-benzyl-3-methylindolin-2-one (6b). To a mixture of substrate $5 \mathrm{~b}(48.2 \mathrm{mg}$, $0.15 \mathrm{mmol}$ ), tris(dibenzylideneacetone)dipalladium(0) $(3.4 \mathrm{mg}, 3.75 \mu \mathrm{mol})$ and $(R, R)$ ANDEN-Phenyl-Trost ligand L6 $(7.9 \mathrm{mg}, 9.75 \mu \mathrm{mol})$ under an argon atmosphere was added dry dimethoxyethane $(3 \mathrm{~mL})$ and the mixture immediately cooled to $-25{ }^{\circ} \mathrm{C}$ with stirring. The mixture was stirred at this temperature for $24 \mathrm{~h}$, then concentrated in vacuo. Flash column chromatography [Petrol:EtOAc 9:1] afforded 6b (39 mg, 94\%) as a bright yellow oil, identical to previously isolated material. ${ }^{5} R_{F} 0.43$ [Petrol:EtOAc 4:1]; 
$\mathrm{V}_{\max }(\mathrm{film}) / \mathrm{cm}^{-1} 2966,2928,1709,1609 ; \delta_{\mathrm{H}}\left(400 \mathrm{MHz}, \mathrm{CDCl}_{3}\right)$ 7.33-7.23 (m, 5H), 7.21 (dd, $J=7.4,1.0 \mathrm{~Hz}, 1 \mathrm{H}), 7.14(\mathrm{td}, J=7.7,1.3 \mathrm{~Hz}, 1 \mathrm{H}), 7.03(\mathrm{td}, J=7.6,1.0 \mathrm{~Hz}, 1 \mathrm{H}), 6.70(\mathrm{~d}, J$ $=7.7 \mathrm{~Hz}, 1 \mathrm{H}$ ), 5.47 (dddd, $J=17.0,10.1,7.9,6.8 \mathrm{~Hz}, 1 \mathrm{H}), 5.05$ (ddd, $J=17.0,3.2,1.3 \mathrm{~Hz}$, $1 \mathrm{H}), 5.02(\mathrm{~d}, J=15.7 \mathrm{~Hz}, 1 \mathrm{H}), 4.95(\mathrm{dd}, J=10.1,2.0 \mathrm{~Hz}, 1 \mathrm{H}), 4.81(\mathrm{~d}, J=15.7 \mathrm{~Hz}, 1 \mathrm{H})$, 2.64 (ddt, $J=13.5,7.9,1.0 \mathrm{~Hz}, 1 \mathrm{H}$ ), 2.57 (ddt, $J=13.5,6.8,1.1 \mathrm{~Hz}, 1 \mathrm{H}), 1.44(\mathrm{~s}, 3 \mathrm{H}$ ); $\delta_{\mathrm{C}}\left(100 \mathrm{MHz}, \mathrm{CDCl}_{3}\right)$ 180.2, 142.2, 136.0, 133.5, 132.6, 128.6, 127.6, 127.4, 127.2, 122.9, 122.3, 118.8, 109.0, 48.3, 43.6, 42.5, 23.2; HRMS (ESI) Found: $[\mathrm{M}+\mathrm{Na}]^{+}, 300.1359$. $\mathrm{C}_{19} \mathrm{H}_{19} \mathrm{NO}$ requires [M+Na] ${ }^{+}$, 300.1359; Chiral HPLC, OJ-R column, $1.0 \mathrm{~mL} / \mathrm{min}, 99: 1$ Hexanes:IPA, $t_{A}($ major $)=10.1 \mathrm{~min}, t_{B}($ minor $)=19.3 \mathrm{~min}, 75 \%$ ee; $[a]_{D}{ }^{25}-20.4$ (c 2.27, $\mathrm{CHCl}_{3}, 75 \%$ ee).

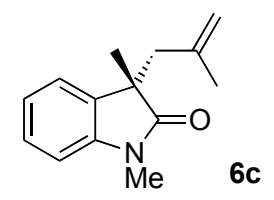

(S)-1,3-Dimethyl-3-(2-methylallyl)indolin-2-one (6c). To a mixture of substrate 5c (39 $\mathrm{mg}, 0.15 \mathrm{mmol})$, tris(dibenzylideneacetone)dipalladium(0) $(3.4 \mathrm{mg}, 3.75 \mu \mathrm{mol})$ and $(R, R)$ ANDEN-Phenyl-Trost ligand L6 $(7.9 \mathrm{mg}, 9.75 \mu \mathrm{mol})$ under an argon atmosphere was added dry tetrahydrofuran $(3 \mathrm{~mL})$ and the mixture immediately cooled to $-25{ }^{\circ} \mathrm{C}$ with stirring. The mixture was stirred at this temperature for $48 \mathrm{~h}$, then concentrated in vacuo. Flash column chromatography [Petrol:EtOAc 14:1-9:1] afforded 6c (21 mg, 65\%, 88\% brsm) as a pale yellow oil. $R_{F} 0.29$ [Petrol:EtOAc 4:1]; $v_{\max }(\mathrm{film}) / \mathrm{cm}^{-1} 2965,1709,1612$; $\delta_{\mathrm{H}}\left(400 \mathrm{MHz}, \mathrm{CDCl}_{3}\right) 7.25(\mathrm{td}, J=7.7,1.3 \mathrm{~Hz}, 1 \mathrm{H}), 7.19(\mathrm{dd}, J=7.4,0.8 \mathrm{~Hz}, 1 \mathrm{H}), 7.05$ (td, $J=7.5,1.0 \mathrm{~Hz}, 1 \mathrm{H}), 6.82(\mathrm{~d}, J=7.8 \mathrm{~Hz}, 1 \mathrm{H}), 4.55(\mathrm{dq}, J=3.1,1.5 \mathrm{~Hz}, 1 \mathrm{H}), 4.48(\mathrm{dq}, J=$ 2.9, $0.9 \mathrm{~Hz}, 1 \mathrm{H}$ ), 3.18 (s, 3H), $2.72(\mathrm{dd}, J=13.5,0.8 \mathrm{~Hz}, 1 \mathrm{H}), 2.47$ (dd, J = 13.5, 0.6 Hz, $1 \mathrm{H}), 1.37(\mathrm{~s}, 3 \mathrm{H}), 1.32(\mathrm{dd}, J=1.4,0.8 \mathrm{~Hz}, 3 \mathrm{H}) ; \delta_{\mathrm{C}}\left(100 \mathrm{MHz}, \mathrm{CDCl}_{3}\right)$ 180.3, 143.2, 141.2, 133.6, 127.7, 123.1, 122.2, 114.2, 107.9, 48.6, 45.6, 26.1, 24.6, 23.5; HRMS (ESI) Found: $[\mathrm{M}+\mathrm{Na}]^{+}$, 238.1202. $\mathrm{C}_{14} \mathrm{H}_{17} \mathrm{NO}$ requires $[\mathrm{M}+\mathrm{Na}]^{+}$, 238.1202; Chiral HPLC, AD column, 1.0 $\mathrm{mL} / \mathrm{min}, 99: 1$ Hexanes:IPA, $t_{A}$ (major) $=8.6 \mathrm{~min}, t_{B}$ (minor) $=10.1 \mathrm{~min}, 89 \%$ ee; $[\mathrm{a}]_{\mathrm{D}}{ }^{25}+4.4$ (c $0.48, \mathrm{CHCl}_{3}, 89 \%$ ee).

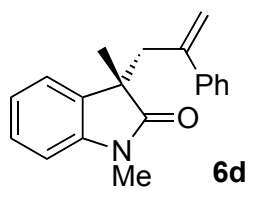


(S)-1,3-Dimethyl-3-(2-phenylallyl)indolin-2-one (6d). To a mixture of substrate $5 \mathbf{d}$ (48.2 $\mathrm{mg}, 0.15 \mathrm{mmol})$, tris(dibenzylideneacetone)dipalladium(0) $(3.4 \mathrm{mg}, 3.75 \mu \mathrm{mol})$ and $(R, R)$ ANDEN-Phenyl-Trost ligand L6 $(7.9 \mathrm{mg}, 9.75 \mu \mathrm{mol})$ under an argon atmosphere was added dry tetrahydrofuran $(3 \mathrm{~mL})$ and the mixture immediately cooled to $-25{ }^{\circ} \mathrm{C}$ with stirring. The mixture was stirred at this temperature for $24 \mathrm{~h}$, then concentrated in vacuo. Flash column chromatography [Petrol:EtOAc 6:1] afforded 6d (40 mg, 96\%) as a yellow oil. $R_{F} 0.27$ [Petrol:EtOAc 4:1]; $\mathrm{v}_{\max }(\mathrm{film}) / \mathrm{cm}^{-1} 2928,1708,1612 ; \delta_{\mathrm{H}}\left(400 \mathrm{MHz}, \mathrm{CDCl}_{3}\right)$ $7.14(\mathrm{td}, J=7.7,1.4 \mathrm{~Hz}, 1 \mathrm{H}), 7.13-7.10(\mathrm{~m}, 3 \mathrm{H}), 6.96$ (ddd, $J=7.4,1.3,0.6 \mathrm{~Hz}, 1 \mathrm{H}), 6.93-$ $6.89(\mathrm{~m}, 2 \mathrm{H}), 6.87(\mathrm{td}, J=7.5,1.0 \mathrm{~Hz}, 1 \mathrm{H}), 6.62(\mathrm{~d}, J=7.7 \mathrm{~Hz}, 1 \mathrm{H}), 4.90(\mathrm{~d}, J=1.7 \mathrm{~Hz}$, $1 \mathrm{H}), 4.87-4.85(\mathrm{~m}, 1 \mathrm{H}), 3.25(\mathrm{dd}, J=13.7,1.1 \mathrm{~Hz}, 1 \mathrm{H}), 2.92(\mathrm{~d}, J=13.7 \mathrm{~Hz}, 1 \mathrm{H}), 2.82(\mathrm{~s}$, $3 \mathrm{H}), 1.36(\mathrm{~s}, 3 \mathrm{H}) ; \delta_{\mathrm{C}}\left(100 \mathrm{MHz}, \mathrm{CDCl}_{3}\right)$ 179.9, 145.7, 143.1, 141.2, 132.7, 127.5, 127.4, 127.0, 126.6, 123.7, 121.8, 116.8, 107.5, 48.5, 43.9, 25.7, 24.0; HRMS (ESI) Found: $[\mathrm{M}+\mathrm{Na}]^{+}, 300.1363 . \mathrm{C}_{19} \mathrm{H}_{19} \mathrm{NO}$ requires $[\mathrm{M}+\mathrm{Na}]^{+}, 300.1359$; Chiral HPLC, OJ-R column, $1.0 \mathrm{~mL} / \mathrm{min}, 95: 5$ Hexanes:IPA, $t_{A}$ (minor) $=9.7 \mathrm{~min}, t_{B}$ (major) $=11.6 \mathrm{~min}, 88 \%$ ee; $[\mathrm{a}]_{\mathrm{D}}{ }^{25}$ $-17.3\left(c 1.03, \mathrm{CHCl}_{3}, 88 \%\right.$ ee) .

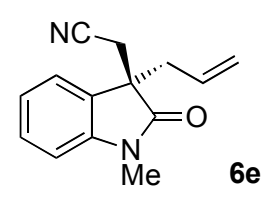

(R)-2-(3-Allyl-1-methyl-2-oxoindolin-3-yl)acetonitrile (6e). To a mixture of substrate 5e (40.5 mg, $0.15 \mathrm{mmol})$, tris(dibenzylideneacetone)dipalladium(0) (3.4 mg, $3.75 \mu \mathrm{mol}$ ) and $(R, R)$-ANDEN-Phenyl-Trost ligand L6 $(7.9 \mathrm{mg}, 9.75 \mu \mathrm{mol})$ under an argon atmosphere was added dry dimethoxyethane $(3 \mathrm{~mL})$ and the mixture immediately cooled to $-25^{\circ} \mathrm{C}$ with stirring. The mixture was stirred at this temperature for $24 \mathrm{~h}$, then concentrated in vacuo. Flash column chromatography [Petrol:EtOAc 4:1] afforded 6 e (34 mg, quant.) as an orange solid, identical to previously isolated material. ${ }^{5} R_{F} 0.31$ [Petrol:EtOAc 2:1]; mp 66$68^{\circ} \mathrm{C} ; \mathrm{v}_{\max }(\mathrm{film}) / \mathrm{cm}^{-1} 2955,2925,1710,1612 ; \delta_{\mathrm{H}}\left(400 \mathrm{MHz}, \mathrm{CDCl}_{3}\right) 7.45$ (ddd, J = 7.4, $1.2,0.5 \mathrm{~Hz}, 1 \mathrm{H}), 7.35$ (td, $J=7.8,1.2 \mathrm{~Hz}, 1 \mathrm{H}), 7.14(\mathrm{td}, J=7.6,1.0 \mathrm{~Hz}, 1 \mathrm{H}), 6.89(\mathrm{~d}, J=$ $7.8 \mathrm{~Hz}, 1 \mathrm{H}$ ), 5.42 (dddd, $J=16.9,10.1,7.8,6.8 \mathrm{~Hz}, 1 \mathrm{H}$ ), 5.09 (ddd, $J=17.0,3.0,1.3 \mathrm{~Hz}$, 1H), 5.00 (ddt, $J=10.1,1.6,0.8 \mathrm{~Hz}, 1 \mathrm{H}$ ), 3.22 (s, 3H), 2.87 (d, J=16.7 Hz, 1H), 2.70 (ddt, $J=13.5,6.8,1.1 \mathrm{~Hz}, 1 \mathrm{H}), 2.67-2.59(\mathrm{~m}, 2 \mathrm{H}) ; \delta_{\mathrm{C}}\left(100 \mathrm{MHz}, \mathrm{CDCl}_{3}\right)$ 176.3, 143.3, 130.5, 129.2, 128.8, 123.4, 123.1, 120.3, 116.4, 108.5, 48.7, 40.2, 26.3, 24.9; HRMS (ESI) Found: $[\mathrm{M}+\mathrm{Na}]^{+}$, 249.1003. $\mathrm{C}_{14} \mathrm{H}_{14} \mathrm{~N}_{2} \mathrm{O}$ requires $[\mathrm{M}+\mathrm{Na}]^{+}, 249.0998$; Chiral HPLC, AD 
column, $1.0 \mathrm{~mL} / \mathrm{min}, 98: 2$ Hexanes:IPA, $t_{A}$ (major) $=21.4 \mathrm{~min}, t_{B}$ (minor) $=25.6 \mathrm{~min}, 68 \%$ ee; $[\mathrm{a}]_{\mathrm{D}}{ }^{25}-39.7$ (c $0.88, \mathrm{CHCl}_{3}, 68 \%$ ee) .

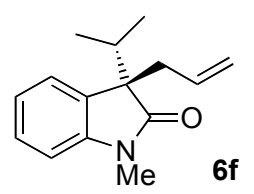

(S)-3-Allyl-3-isopropyl-1-methylindolin-2-one (6f). To a mixture of substrate $5 f$ (41 $\mathrm{mg}$, $0.15 \mathrm{mmol}$ ), tris(dibenzylideneacetone)dipalladium(0) $(3.4 \mathrm{mg}, 3.75 \mu \mathrm{mol})$ and $(R, R)$ ANDEN-Phenyl-Trost ligand L6 $(7.9 \mathrm{mg}, 9.75 \mu \mathrm{mol})$ under an argon atmosphere was added dry tetrahydrofuran $(3 \mathrm{~mL})$ and the mixture immediately cooled to $-25{ }^{\circ} \mathrm{C}$ with stirring. The mixture was stirred at this temperature for $24 \mathrm{~h}$, then concentrated in vacuo. Flash column chromatography [Petrol:EtOAc 9:1] afforded $6 f(30 \mathrm{mg}, 87 \%)$ as a clear oil, identical to previously isolated material. ${ }^{5} R_{F} 0.43$ [Petrol:EtOAc 4:1]; $\mathrm{v}_{\max }(\mathrm{film}) / \mathrm{cm}^{-1} 3074$, 2964, 1704, 1610; $\delta_{\mathrm{H}}\left(400 \mathrm{MHz}, \mathrm{CDCl}_{3}\right) 7.26$ (td, $\left.J=7.7,1.3 \mathrm{~Hz}, 1 \mathrm{H}\right), 7.19$ (dd, $J=7.4$, $0.8 \mathrm{~Hz}, 1 \mathrm{H}), 7.04(\mathrm{td}, J=7.5,1.0 \mathrm{~Hz}, 1 \mathrm{H}), 6.80(\mathrm{~d}, J=7.8 \mathrm{~Hz}, 1 \mathrm{H}), 5.36-5.24(\mathrm{~m}, 1 \mathrm{H}), 4.93$ (ddd, $J=17.0,3.3,1.3 \mathrm{~Hz}, 1 \mathrm{H}$ ), 4.81 (ddt, $J=10.1,2.0,1.0 \mathrm{~Hz}, 1 \mathrm{H}$ ), 3.17 (s, 3H), 2.64 (ddt, $J=13.5,6.8,1.1 \mathrm{~Hz}, 1 \mathrm{H}$ ), 2.59 (ddt, $J=13.7,8.0,1.2 \mathrm{~Hz}, 1 \mathrm{H}$ ), 2.19 (sept, $J=6.9 \mathrm{~Hz}$, $1 \mathrm{H}), 0.97(\mathrm{~d}, J=6.9 \mathrm{~Hz}, 3 \mathrm{H}), 0.72(\mathrm{~d}, J=6.8 \mathrm{~Hz}, 3 \mathrm{H}) ; \delta_{\mathrm{C}}\left(100 \mathrm{MHz}, \mathrm{CDCl}_{3}\right)$ 179.4, 144.2, 132.8, 130.7, 127.6, 123.7, 122.0, 118.2, 107.5, 56.2, 39.5, 34.7, 25.8, 17.3, 17.2; HRMS (ESI) Found: $[\mathrm{M}+\mathrm{H}]^{+}, 230.1541 . \mathrm{C}_{15} \mathrm{H}_{19} \mathrm{NO}$ requires $[\mathrm{M}+\mathrm{H}]^{+}, 230.1539$; Chiral HPLC, AD column, 0.5 mL/min, 99.7:0.3 Hexanes:IPA, $t_{A}$ (minor) $=35.7 \mathrm{~min}, t_{B}$ (major) = $38.0 \mathrm{~min}$, $72 \%$ ee (approximate value as baseline separation could not be achieved); $[a]_{D}^{25}+36.3(c$ $0.75, \mathrm{CHCl}_{3}, 72 \%$ ee).

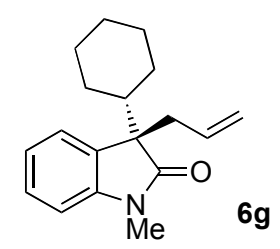

(S)-3-Allyl-3-cyclohexyl-1-methylindolin-2-one $(\mathbf{6 g})$. To a mixture of substrate $\mathbf{5 g}$ (47 $\mathrm{mg}, 0.15 \mathrm{mmol})$, tris(dibenzylideneacetone)dipalladium(0) $(3.4 \mathrm{mg}, 3.75 \mu \mathrm{mol})$ and $(R, R)$ ANDEN-Phenyl-Trost ligand L6 $(7.9 \mathrm{mg}, 9.75 \mu \mathrm{mol})$ under an argon atmosphere was added dry tetrahydrofuran $(3 \mathrm{~mL})$ and the mixture immediately cooled to $-25{ }^{\circ} \mathrm{C}$ with stirring. The mixture was stirred at this temperature for $24 \mathrm{~h}$, then concentrated in vacuo. Flash column chromatography [Petrol:EtOAc 19:1] afforded $6 \mathrm{~g}$ (40.5 mg, quant.) as a 
clear oil. $R_{F} 0.48$ [Petrol:EtOAc 4:1]; $v_{\max }(\mathrm{film}) / \mathrm{cm}^{-1} 2928,2859,1714,1609 ; \delta_{\mathrm{H}}(400$ $\mathrm{MHz}_{\mathrm{CDCl}}$ ) $7.24(\mathrm{td}, J=7.7,1.3 \mathrm{~Hz}, 1 \mathrm{H}), 7.18(\mathrm{dd}, J=7.4,0.7 \mathrm{~Hz}, 1 \mathrm{H}), 7.03$ (td, $J=7.5$, $1.0 \mathrm{~Hz}, 1 \mathrm{H}$ ), 6.78 (d, J=7.7 Hz, 1H), 5.27 (dddd, $J=16.7,10.1,7.7,6.8 \mathrm{~Hz}, 1 \mathrm{H}$ ), 4.91 (ddd, $J=17.0,3.3,1.3 \mathrm{~Hz}, 1 \mathrm{H}$ ), 4.79 (ddt, $J=10.1,2.1,1.1 \mathrm{~Hz}, 1 \mathrm{H}$ ), 3.15 (s, 3H), 2.63 (ddt, $J=13.3,6.8,1.1 \mathrm{~Hz}, 1 \mathrm{H}), 2.56$ (ddt, $J=13.6,8.0,1.1 \mathrm{~Hz}, 1 \mathrm{H}$ ), 1.85 (tt, $J=11.8,2.9$ $\mathrm{Hz}, 1 \mathrm{H}), 1.78-1.68(\mathrm{~m}, 2 \mathrm{H}), 1.63-1.53(\mathrm{~m}, 2 \mathrm{H}), 1.47(\mathrm{~d}, J=12.7 \mathrm{~Hz}, 1 \mathrm{H}), 1.25-1.10(\mathrm{~m}$, $3 \mathrm{H}$ ), 1.00 (qt, $J=12.8,3.7 \mathrm{~Hz}, 1 \mathrm{H}), 0.81$ (qd, $J=12.8,3.6 \mathrm{~Hz}, 1 \mathrm{H}) ; \delta_{\mathrm{C}}\left(100 \mathrm{MHz}, \mathrm{CDCl}_{3}\right)$ 179.4, 144.0, 132.7, 131.2, 127.4, 123.7, 121.9, 118.1, 107.4, 56.3, 44.7, 38.9, 27.3, 27.1,

26.5, 26.2, 26.1, 25.7; HRMS (ESI) Found: $[\mathrm{M}+\mathrm{H}]^{+}$, 270.1853. $\mathrm{C}_{18} \mathrm{H}_{23} \mathrm{NO}$ requires [M+H] $]^{+}$, 270.1852; Chiral HPLC, OJ-R column, $0.5 \mathrm{~mL} / \mathrm{min}, 99: 1$ Hexanes:IPA, $t_{A}$ (major) = 7.1 $\min , t_{B}($ minor $)=8.0 \mathrm{~min}, 76 \%$ ee; $[\mathrm{a}]_{\mathrm{D}}^{25}+53.0\left(c 1.07, \mathrm{CHCl}_{3}, 76 \%\right.$ ee $)$.

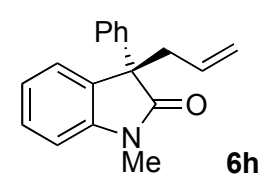

(S)-3-Allyl-1-methyl-3-phenylindolin-2-one (6h). To a mixture of substrate $5 \mathrm{~h} \mathrm{(46} \mathrm{mg}$, $0.15 \mathrm{mmol})$, tris(dibenzylideneacetone)dipalladium(0) $(3.4 \mathrm{mg}, 3.75 \mu \mathrm{mol})$ and $(R, R)$ ANDEN-Phenyl-Trost ligand L6 $(7.9 \mathrm{mg}, 9.75 \mu \mathrm{mol})$ under an argon atmosphere was added dry toluene $(3 \mathrm{~mL})$ and the mixture immediately cooled to $-25^{\circ} \mathrm{C}$ with stirring. The mixture was stirred at this temperature for $24 \mathrm{~h}$, then concentrated in vacuo. Flash column chromatography [Petrol:EtOAc 6:1] afforded 6h (39 mg, 99\%) as a clear sticky oil, identical to previously isolated material. ${ }^{6} R_{F} 0.25$ [Petrol:EtOAc 4:1]; $\mathrm{v}_{\max }(\mathrm{film}) / \mathrm{cm}^{-1} 2928,1706$, 1609; $\delta_{\mathrm{H}}\left(400 \mathrm{MHz}, \mathrm{CDCl}_{3}\right)$ 7.42-7.38 (m, 2H), 7.36-7.21 (m, 5H), 7.12 (td, J=7.5, 1.0 Hz, $1 \mathrm{H}$ ), 6.90 (d, $J=7.8 \mathrm{~Hz}, 1 \mathrm{H}$ ), 5.41 (ddt, $J=17.2,10.1,7.2 \mathrm{~Hz}, 1 \mathrm{H}$ ), 5.04 (ddd, $J=17.0$, 3.2, $1.3 \mathrm{~Hz}, 1 \mathrm{H}$ ), 4.93 (ddt, $J=10.1,1.8,0.8 \mathrm{~Hz}, 1 \mathrm{H}$ ), 3.20 (s, 3H), 3.04 (dt, J = 7.2, 1.0 $\mathrm{Hz}, 2 \mathrm{H}) ; \delta_{\mathrm{C}}\left(100 \mathrm{MHz}, \mathrm{CDCl}_{3}\right)$ 177.8, 143.7, 139.4, 132.3, 131.5, 128.4, 128.1, 127.2, 126.9, 125.1, 122.4, 119.1, 108.1, 56.3, 41.9, 26.2; HRMS (ESI) Found: [M+H] ${ }^{+}, 264.1384$. $\mathrm{C}_{18} \mathrm{H}_{17} \mathrm{NO}$ requires $[\mathrm{M}+\mathrm{H}]^{+}, 264.1383$; Chiral HPLC, OJ-R column, $1.0 \mathrm{~mL} / \mathrm{min}, 90: 10$ Hexanes:IPA, $t_{A}$ (major) $=6.0 \mathrm{~min}, t_{B}$ (minor) $=22.8 \mathrm{~min}, 79 \%$ ee; $[\mathrm{a}]_{\mathrm{D}}{ }^{25}-124.2$ (c 0.97 , $\mathrm{CHCl}_{3}, 79 \%$ ee).

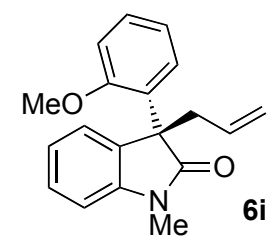


(R)-3-Allyl-3-(2-methoxyphenyl)-1-methylindolin-2-one (6i). To a mixture of substrate $\mathbf{5 i}$ (50.6 mg, $0.15 \mathrm{mmol}$ ), tris(dibenzylideneacetone)dipalladium(0) $(3.4 \mathrm{mg}, 3.75 \mu \mathrm{mol})$ and $(R, R)$-ANDEN-Phenyl-Trost ligand $\mathbf{L} 6(7.9 \mathrm{mg}, 9.75 \mu \mathrm{mol})$ under an argon atmosphere was added dry toluene $(3 \mathrm{~mL})$ and the mixture immediately cooled to $-25^{\circ} \mathrm{C}$ with stirring. The mixture was stirred at this temperature for $24 \mathrm{~h}$, then concentrated in vacuo. Flash column chromatography [Petrol:EtOAc 4:1] afforded $6 \mathbf{i}$ (44 mg, quant.) as a yellow solid, identical to previously isolated material. ${ }^{6} R_{F} 0.25$ [Petrol:EtOAc 2:1]; mp 88-90 ${ }^{\circ} \mathrm{C}$; $\mathrm{V}_{\max }(\mathrm{film}) / \mathrm{cm}^{-1} 2937,1708 ; \delta_{\mathrm{H}}\left(400 \mathrm{MHz}, \mathrm{CDCl}_{3}\right) 7.57$ (dd, $\left.J=7.8,1.6 \mathrm{~Hz}, 1 \mathrm{H}\right), 7.27-7.19$ (m, 2H), $7.03(\mathrm{td}, J=7.6,1.2 \mathrm{~Hz}, 1 \mathrm{H}), 6.93(\mathrm{td}, J=7.5,1.0 \mathrm{~Hz}, 1 \mathrm{H}), 6.86(\mathrm{dd}, J=7.3,1.4$ $\mathrm{Hz}, 1 \mathrm{H}), 6.82(\mathrm{~d}, J=7.7 \mathrm{~Hz}, 1 \mathrm{H}), 6.76(\mathrm{dd}, J=8.2,1.1 \mathrm{~Hz}, 1 \mathrm{H}$ ), 5.35 (ddt, $J=17.2,10.1$, $7.1 \mathrm{~Hz}, 1 \mathrm{H}$ ), $5.00(\mathrm{dq}, J=17.0,1.3 \mathrm{~Hz}, 1 \mathrm{H}$ ), 4.88 (ddt, $J=10.1,1.9,0.9 \mathrm{~Hz}, 1 \mathrm{H}$ ), 3.41 (s, $3 \mathrm{H}$ ), 3.27 (s, 3H), 3.00 (dt, $J=7.1,1.0 \mathrm{~Hz}, 2 \mathrm{H})$; $\delta_{\mathrm{C}}\left(100 \mathrm{MHz}, \mathrm{CDCl}_{3}\right) 179.2,157.1,144.3$, 133.2, 131.7, 129.6, 128.6, 127.4, 127.4, 122.5, 122.0, 120.8, 119.0, 112.2, 107.0, 55.8, 53.7, 40.5, 26.1; HRMS (ESI) Found: $[\mathrm{M}+\mathrm{H}]^{+}, 294.1478 . \mathrm{C}_{19} \mathrm{H}_{19} \mathrm{NO}_{2}$ requires $[\mathrm{M}+\mathrm{H}]^{+}$, 294.1489; Chiral HPLC, OJ-R column, $1.0 \mathrm{~mL} / \mathrm{min}, 90: 10$ Hexanes:IPA, $t_{A}$ (major) $=14.7$ $\min , t_{B}$ (minor) $=35.6 \mathrm{~min}, 95 \%$ ee; $[\mathrm{a}]_{D}{ }^{25}+77.0\left(c 1.38, \mathrm{CHCl}_{3}, 95 \%\right.$ ee $)$.

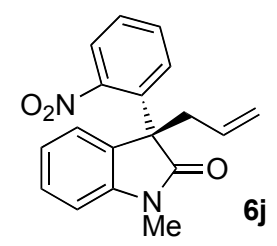

(R)-3-Allyl-1-methyl-3-(2-nitrophenyl)indolin-2-one (6j). To a mixture of substrate $\mathbf{5 j}$ (35.2 $\mathrm{mg}, 0.1 \mathrm{mmol}$ ), tris(dibenzylideneacetone)dipalladium(0) (2.3 $\mathrm{mg}, 2.5 \mu \mathrm{mol}$ ) and $(R, R)$-ANDEN-Phenyl-Trost ligand $\mathbf{L} 6(5.3 \mathrm{mg}, 6.5 \mu \mathrm{mol})$ under an argon atmosphere was added dry toluene $(2 \mathrm{~mL})$ and the mixture immediately cooled to $-25^{\circ} \mathrm{C}$ with stirring. The mixture was stirred at this temperature for $24 \mathrm{~h}$, then concentrated in vacuo. Flash column chromatography [Petrol:EtOAc 4:1] afforded $6 \mathbf{j}$ (31 mg, quant.) as a yellow solid. $R_{F} 0.31$ [Petrol:EtOAc 2:1]; $\mathrm{mp} 132-133^{\circ} \mathrm{C} ; \mathrm{v}_{\max }(\mathrm{film}) / \mathrm{cm}^{-1} 3074,1703,1610,1524,1365 ; \delta_{\mathrm{H}}(400$ $\left.\mathrm{MHz}, \mathrm{CDCl}_{3}\right) 7.93$ (dd, $J=8.1,1.0 \mathrm{~Hz}, 1 \mathrm{H}$ ), $7.68(\mathrm{td}, J=8.0,1.5 \mathrm{~Hz}, 1 \mathrm{H}), 7.59$ (dd, $J=8.0$, $1.4 \mathrm{~Hz}, 1 \mathrm{H}$ ), 7.44 (td, $J=8.0,1.2 \mathrm{~Hz}, 1 \mathrm{H}$ ), 7.31 (td, $J=7.7,1.3 \mathrm{~Hz}, 1 \mathrm{H}), 6.99$ (td, $J=7.6$, $0.9 \mathrm{~Hz}, 1 \mathrm{H}$ ), 6.89 (dd, $J=7.4,0.7 \mathrm{~Hz}, 1 \mathrm{H}$ ), $6.86(\mathrm{~d}, J=7.8 \mathrm{~Hz}, 1 \mathrm{H}$ ), 5.36 (ddt, $J=17.0$, $10.1,7.0 \mathrm{~Hz}, 1 \mathrm{H}$ ), 5.01 (ddt, $J=17.0,1.6,1.2 \mathrm{~Hz}, 1 \mathrm{H}), 4.98-4.94(\mathrm{~m}, 1 \mathrm{H}), 3.27(\mathrm{~s}, 3 \mathrm{H})$, $3.00(\mathrm{~d}, J=6.8 \mathrm{~Hz}, 2 \mathrm{H}) ; \delta_{\mathrm{C}}\left(100 \mathrm{MHz}, \mathrm{CDCl}_{3}\right) 177.2,150.3,144.3,132.2,132.1,130.8$, $130.5,130.3,128.8,128.5,124.9,122.8,122.4,120.0,108.1,54.4,42.8,26.3$; HRMS 
(ESI) Found: $[\mathrm{M}+\mathrm{Na}]^{+}, 331.1056 . \mathrm{C}_{18} \mathrm{H}_{16} \mathrm{~N}_{2} \mathrm{O}_{3}$ requires $[\mathrm{M}+\mathrm{Na}]^{+}, 331.1053$; Chiral HPLC, OJ-R column, $1.0 \mathrm{~mL} / \mathrm{min}, 90: 10$ Hexanes:IPA, $t_{A}$ (major) $=15.5 \mathrm{~min}, t_{B}$ (minor) $=26.4$ $\min , 87 \%$ ee; $[\mathrm{a}]_{\mathrm{D}}^{25}-130.3$ (c $0.83, \mathrm{CHCl}_{3}, 87 \%$ ee).

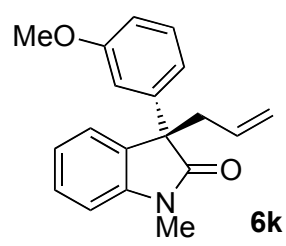

(S)-3-Allyl-3-(3-methoxyphenyl)-1-methylindolin-2-one (6k). To a mixture of substrate 5k (50.6 mg, $0.15 \mathrm{mmol}$ ), tris(dibenzylideneacetone)dipalladium(0) (3.4 mg, $3.75 \mu \mathrm{mol}$ ) and $(R, R)$-ANDEN-Phenyl-Trost ligand $\mathbf{L} 6(7.9 \mathrm{mg}, 9.75 \mu \mathrm{mol})$ under an argon atmosphere was added dry toluene $(3 \mathrm{~mL})$ and the mixture immediately cooled to $-25^{\circ} \mathrm{C}$ with stirring. The mixture was stirred at this temperature for $24 \mathrm{~h}$, then concentrated in vacuo. Flash column chromatography [Petrol:EtOAc 6:1] afforded 6k (40 mg, 91\%) as a clear oil. $R_{F}$ 0.24 [Petrol:EtOAc 4:1]; $\mathrm{v}_{\max }(\mathrm{film}) / \mathrm{cm}^{-1} 2937,1709,1608 ; \delta_{\mathrm{H}}\left(400 \mathrm{MHz}, \mathrm{CDCl}_{3}\right) 7.33$ (td, $J$ $=7.7,1.2 \mathrm{~Hz}, 1 \mathrm{H}$ ), 7.27 (dd, $J=7.0,0.7 \mathrm{~Hz}, 1 \mathrm{H}), 7.22(\mathrm{t}, J=8.0 \mathrm{~Hz}, 1 \mathrm{H}), 7.11$ (td, $J=7.5$, $0.9 \mathrm{~Hz}, 1 \mathrm{H}), 7.00-6.94(\mathrm{~m}, 2 \mathrm{H}), 6.89(\mathrm{~d}, J=7.8 \mathrm{~Hz}, 1 \mathrm{H}), 6.79$ (ddd, $J=8.3,2.4,0.7 \mathrm{~Hz}$, $1 \mathrm{H}$ ), 5.40 (ddt, $J=17.1,10.1,7.1 \mathrm{~Hz}, 1 \mathrm{H}$ ), 5.03 (ddd, $J=17.0,3.0,1.2 \mathrm{~Hz}, 1 \mathrm{H}$ ), 4.92 (dd, $J$ $=10.1,1.9 \mathrm{~Hz}, 1 \mathrm{H}), 3.77(\mathrm{~s}, 3 \mathrm{H}), 3.20(\mathrm{~s}, 3 \mathrm{H}), 3.04-3.00(\mathrm{~m}, 2 \mathrm{H}) ; \delta_{\mathrm{C}}\left(100 \mathrm{MHz}, \mathrm{CDCl}_{3}\right)$ 177.8, 159.6, 143.7, 141.0, 132.4, 131.5, 129.4, 128.2, 125.1, 122.4, 119.4, 119.1, 113.5, 112.2, 108.2, 56.3, 55.1, 41.9, 26.3; HRMS (ESI) Found: $[\mathrm{M}+\mathrm{H}]^{+}$, 294.1490. $\mathrm{C}_{19} \mathrm{H}_{19} \mathrm{NO}_{2}$ requires $[\mathrm{M}+\mathrm{H}]^{+}$, 294.1489; Chiral HPLC, OJ-R column, $1.0 \mathrm{~mL} / \mathrm{min}, 90: 10$ Hexanes:IPA, $t_{A}$ $($ major $)=7.4 \mathrm{~min}, t_{B}($ minor $)=16.7 \mathrm{~min}, 78 \%$ ee; $[\mathrm{a}]_{D}^{25}-90.7\left(c 1.13, \mathrm{CHCl}_{3}, 78 \%\right.$ ee $)$. 


\section{Comparison of Optical Rotation Data}

\begin{tabular}{|c|c|c|c|c|c|}
\hline Entry & Product & $\begin{array}{c}\text { Optical rotation (in } \mathrm{CHCl}_{3} \text { ) } \\
\text { Measured }\end{array}$ & $\begin{array}{c}\text { Optical rotation (in } \mathrm{CHCl}_{3} \text { ) } \\
\text { Literature values }\end{array}$ & Reference & A-values \\
\hline 1 & & $\begin{array}{c}{[a]_{D}^{25}-6.2} \\
(c 0.62,74 \% \text { ee })\end{array}$ & $\begin{array}{c}{[a]_{D}^{24}-9.7} \\
(c 0.9,81 \% \text { ee })\end{array}$ & Trost $^{5}$ & 1.74 \\
\hline 2 & & $\begin{array}{c}{[\mathrm{a}]_{\mathrm{D}}^{25}-20.4} \\
(c 2.27,75 \% \text { ee })\end{array}$ & $\begin{array}{c}{[a]_{D}^{24}-26.9} \\
(c 1.5,75 \% \text { ee })\end{array}$ & Trost $^{5}$ & 1.74 \\
\hline 3 & & $\begin{array}{c}{[a]_{D}^{25}+4.4} \\
(c 0.48,89 \% \text { ee })\end{array}$ & - & - & 1.74 \\
\hline 4 & & $\begin{array}{c}{[\mathrm{a}]_{\mathrm{D}}^{25}-17.3} \\
(c 1.03,88 \% \text { ee })\end{array}$ & - & - & 1.74 \\
\hline 5 & & $\begin{array}{c}{[a]_{D}^{25}-39.7} \\
(c 0.88,68 \% \text { ee })\end{array}$ & $\begin{array}{c}{[a]_{D}^{24}-40.9} \\
(c 1.0,93 \% \text { ee })\end{array}$ & Trost $^{5}$ & 1.77 \\
\hline 6 & & $\begin{array}{c}{[a]_{D}^{25}+36.3} \\
(c 0.75,72 \% \text { ee })\end{array}$ & $\begin{array}{c}\text { enantiomer: } \\
{[a]_{D}^{24}-57.7} \\
(c 2.0,91 \% \text { ee })\end{array}$ & Trost $^{5}$ & 2.21 \\
\hline 7 & & $\begin{array}{c}{[\mathrm{a}]_{\mathrm{D}}^{25}+53.0} \\
(c 1.07,76 \% \text { ee })\end{array}$ & - & - & 2.2 \\
\hline 8 & & $\begin{array}{c}{[\mathrm{a}]_{\mathrm{D}}^{25}-124.2} \\
(c 0.97,79 \% \mathrm{ee})\end{array}$ & $\begin{array}{c}{[\mathrm{a}]_{\mathrm{D}}^{20}-160.7} \\
(c 0.30,>99 \% \text { ee }) \\
\text { enantiomer: } \\
{[\mathrm{a}]_{\mathrm{D}}^{27}+118.3} \\
(c 1.0,81 \% \text { ee })\end{array}$ & $\begin{array}{l}\text { Smith }^{7} \\
\text { Dorta }^{8}\end{array}$ & 2.8 \\
\hline 9 & & $\begin{array}{c}{[a]_{D}^{25}+77.0} \\
(c 1.38,95 \% \text { ee })\end{array}$ & $\begin{array}{c}\text { enantiomer: } \\
{[\mathrm{a}]_{\mathrm{D}}^{27}-86.8} \\
(c 1.0,83 \% \text { ee })\end{array}$ & Dorta $^{8}$ & $>2.8$ \\
\hline 10 & & $\begin{array}{c}{[a]_{D}^{25}-130.3} \\
(c 0.83,87 \% \text { ee })\end{array}$ & - & - & $>2.8$ \\
\hline 11 & & $\begin{array}{c}{[\mathrm{a}]_{\mathrm{D}}^{25}-90.7} \\
(c 1.13,78 \% \text { ee })\end{array}$ & $\begin{array}{c}\text { enantiomer: } \\
{[\mathrm{a}]_{\mathrm{D}}^{27}+100.3} \\
(c 0.5,85 \% \text { ee })\end{array}$ & Dorta $^{8}$ & $>2.8$ \\
\hline
\end{tabular}




\section{References}

(1) Navarro, I.; Basset, J. F.; Hebbe, S.; Major, S. M.; Werner, T.; Howsham, C.; Brackow, J.; Barrett, A. G. M. J. Am. Chem. Soc. 2008, 130, 10293-10298.

(2) Duan, Z. C.; Hu, X. P.; Zhang, C.; Wang, D. Y.; Yu, S. B.; Zheng, Z. J. Org. Chem. 2009, 74, 9191-9194.

(3) Motoyama, Y.; Aoki, M.; Takaoka, N.; Aoto, R.; Nagashima, H. Chem. Commun. 2009, 1574-1576.

(4) Makosza, M.; Sypniewski, M.; Glinka, T. Tetrahedron 1996, 52, 3189-3194.

(5) Trost, B. M.; Zhang, Y. J. Am. Chem. Soc. 2006, 128, 4590-4591.

(6) Trost, B. M.; Frederiksen, M. U. Angew. Chem. Int. Ed. 2005, 44, 308-310.

(7) Duguet, N.; Slawin, A. M. Z.; Smith, A. D. Org. Lett. 2009, 11, 3858-3861.

(8) Luan, X. J.; Wu, L. L.; Drinkel, E.; Mariz, R.; Gatti, M.; Dorta, R. Org. Lett. 2010, 12, 1912-1915. 


\section{HPLC Data}

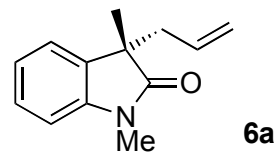

a) racemate

AD column; 99: 1 Hex/IPA, 1 mL/min, 23 bar

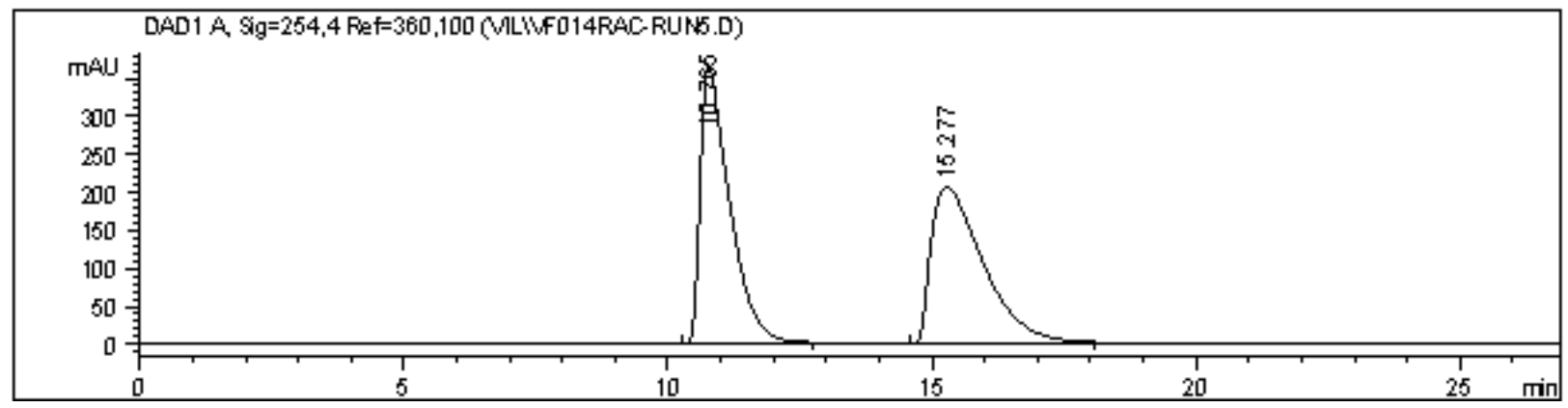

Signal 1: DADl A, Sig $=254,4$ Ref $=360,100$

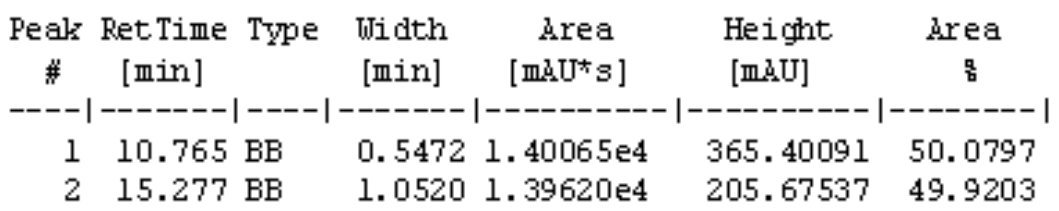

b) $74 \% \mathrm{ee}$

AD column, 99: 1 Hex: IPA, 1 mL/min, 24 bar

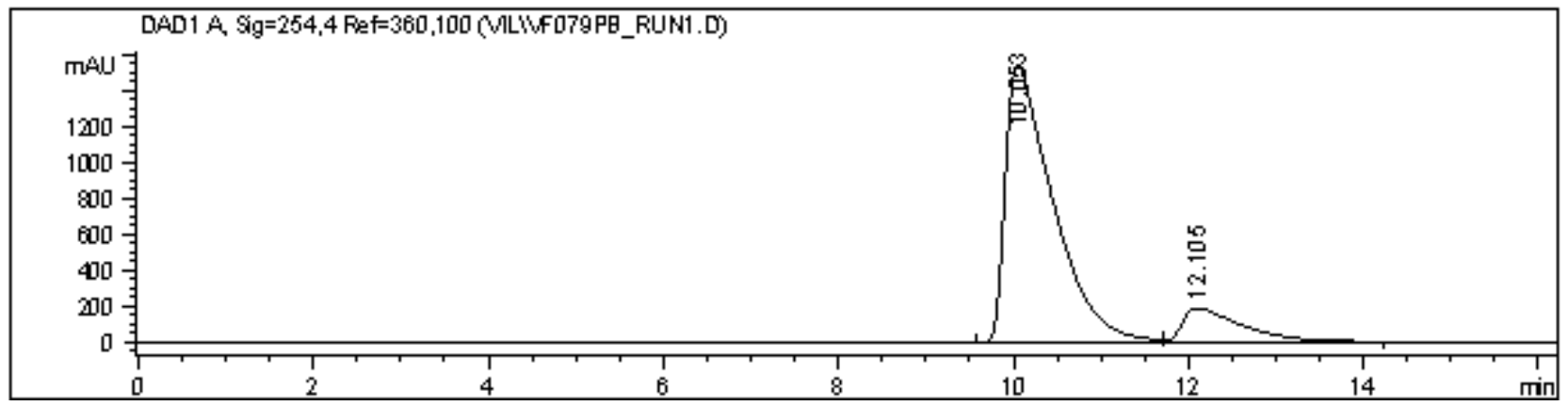

Signal 1: DADl A, Sig $=254,4$ Ref $=360,100$

\begin{tabular}{|c|c|c|c|c|c|c|}
\hline $\begin{array}{c}\text { Pealz } \\
\#\end{array}$ & $\begin{array}{l}\text { Ret Time } \\
\text { [min] }\end{array}$ & Type & $\begin{array}{l}\text { Width } \\
\text { [min] }\end{array}$ & $\begin{array}{c}\text { Area } \\
\text { [meUts }\end{array}$ & $\begin{array}{l}\text { Height } \\
\text { [miU] }\end{array}$ & $\begin{array}{c}\text { Area } \\
\text { 몸 }\end{array}$ \\
\hline & 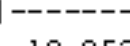 & & - & ---------- & |----------- & \\
\hline $\begin{array}{l}1 \\
2\end{array}$ & $\begin{array}{l}10.053 \\
12.105\end{array}$ & $\begin{array}{l}\text { BV } \\
\text { VB }\end{array}$ & $\begin{array}{l}0.5308 \\
0.6665\end{array}$ & $\begin{array}{l}5.80730 \mathrm{e} 4 \\
8834.87109\end{array}$ & $\begin{array}{r}1544.03931 \\
188.60004\end{array}$ & $\begin{array}{l}86.795 .5 \\
13.2045\end{array}$ \\
\hline
\end{tabular}




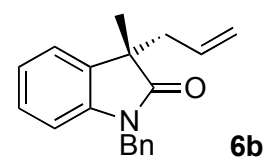

a) racemate

0J-R column, 99:1 Hex:IPA $1 \mathrm{~mL} / \min , 30$ bar

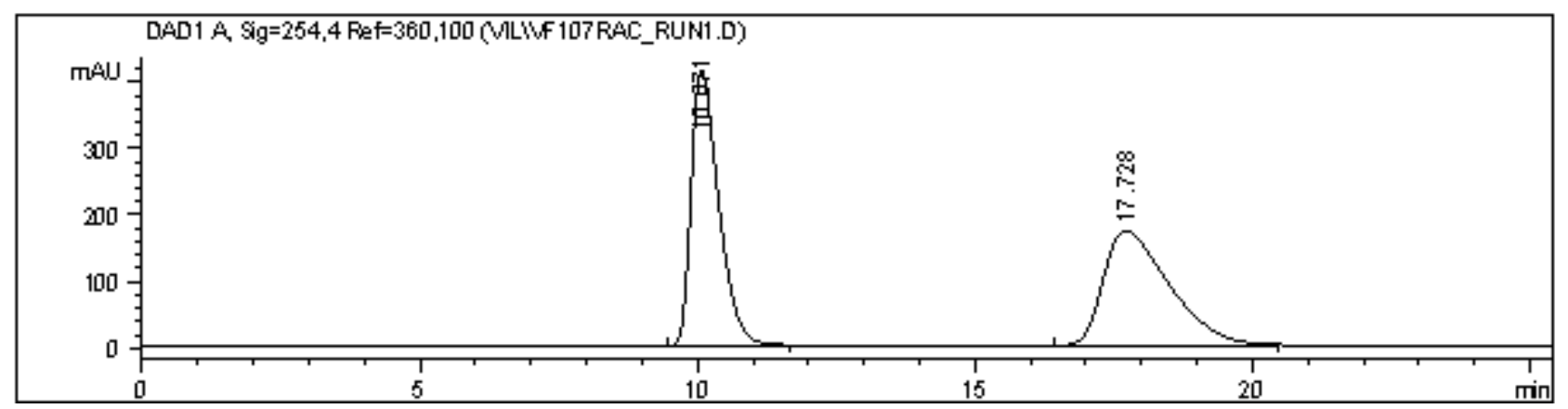

Signal 1: DADl A, Sig $=254,4$ Ref $=360,100$

\begin{tabular}{|c|c|c|c|c|c|}
\hline $\begin{array}{c}\text { Peak } \\
\#\end{array}$ & $\begin{array}{l}\text { RetTime Type } \\
\text { [min] }\end{array}$ & $\begin{array}{l}\text { Width } \\
\text { [min] }\end{array}$ & $\begin{array}{c}\text { Area } \\
{\left[\mathrm{ma}^{2} \mathrm{U}^{*} \mathrm{~s}\right]}\end{array}$ & $\begin{array}{l}\text { Height } \\
\text { [miU] }\end{array}$ & $\begin{array}{c}\text { Area } \\
\text { 몹 }\end{array}$ \\
\hline- & & ----- & -------- & $-0-0-0-0$ & ------ \\
\hline 1 & $10.071 \mathrm{BB}$ & 0.5217 & 1. $41959 \mathrm{e} 4$ & 414.39221 & 50.0798 \\
\hline 2 & $28 \mathrm{BB}$ & 326 & $1.41 .506 \mathrm{e} 4$ & 172.22816 & 49.9202 \\
\hline
\end{tabular}

b) $75 \%$ ee

0J-R column, 99:1 Hex:IPA, $1 \mathrm{~mL} / \min , 29$ bar

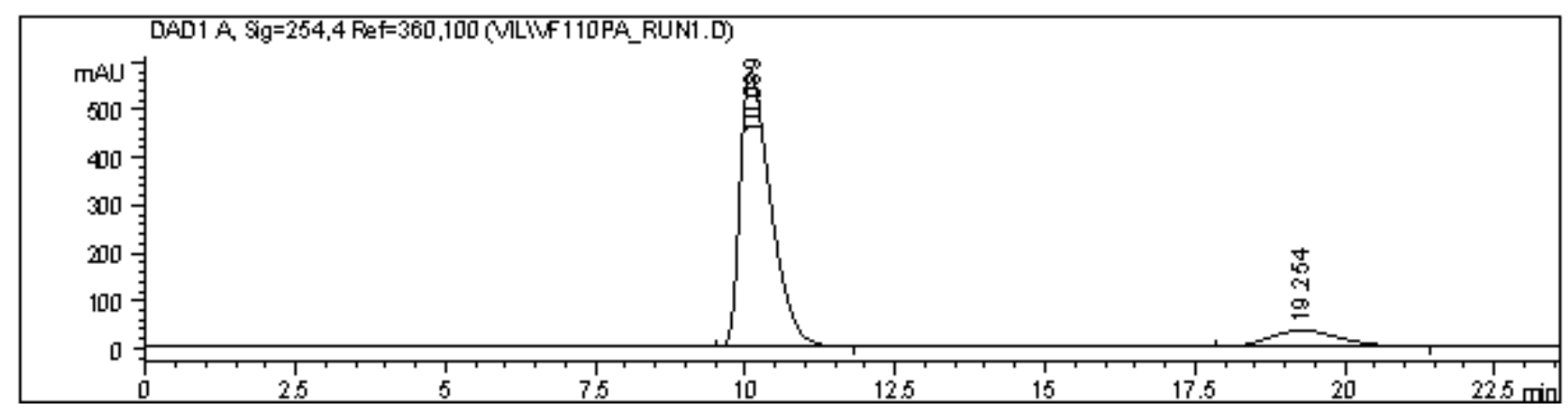

Signal 1: DADl A, Sig $=254,4$ Ref $=360,100$

\begin{tabular}{|c|c|c|c|c|c|}
\hline $\begin{array}{c}\text { Peak } \\
\#\end{array}$ & $\begin{array}{l}\text { Ret Time Type } \\
\text { [min] }\end{array}$ & $\begin{array}{l}\text { Width } \\
\text { [min] }\end{array}$ & $\begin{array}{c}\text { Area } \\
\text { [mind*'s] }\end{array}$ & $\begin{array}{l}\text { Height } \\
\text { [mbU] }\end{array}$ & $\begin{array}{c}\text { Area } \\
\text { 믑 }\end{array}$ \\
\hline & & & & & \\
\hline 2 & $4 \mathrm{BB}$ & & 2868.74390 & 34.90999 & 12.2760 \\
\hline
\end{tabular}




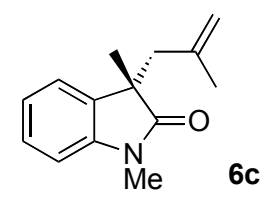

a) racemate

AD column, 99: 1 Hex: IPA $1 \mathrm{~mL} / \mathrm{min}, 24 \mathrm{bar}$

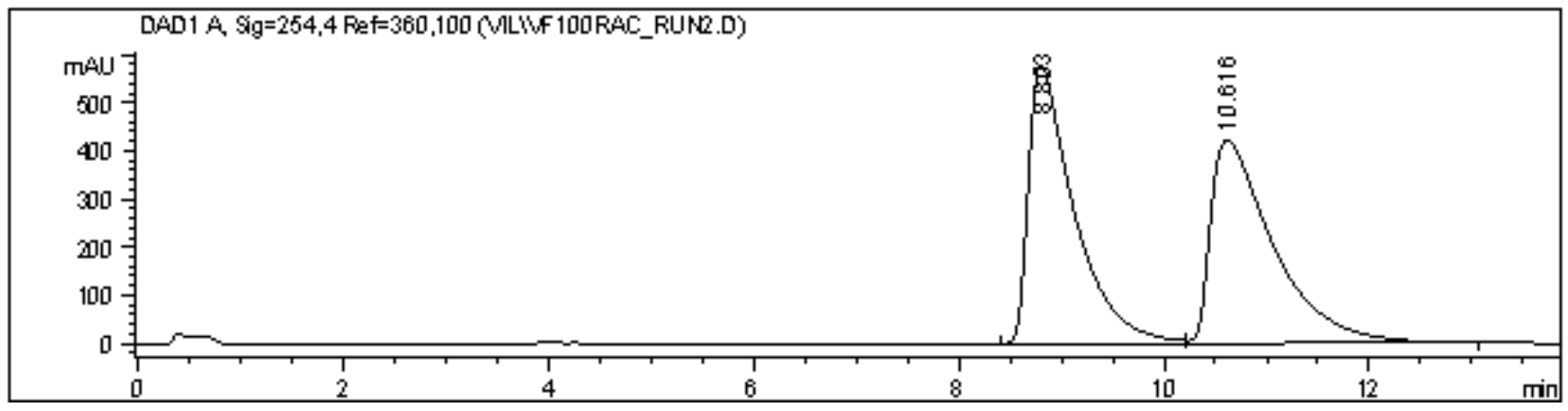

Signal 1: DADl A, Sig=254,4 Ref=360, 100

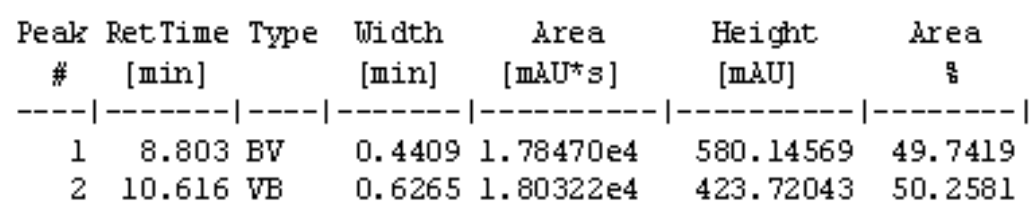

b) $89 \%$ ee

AD column, 99: 1 Hex:IPA, 1 mL/min, 24 bar

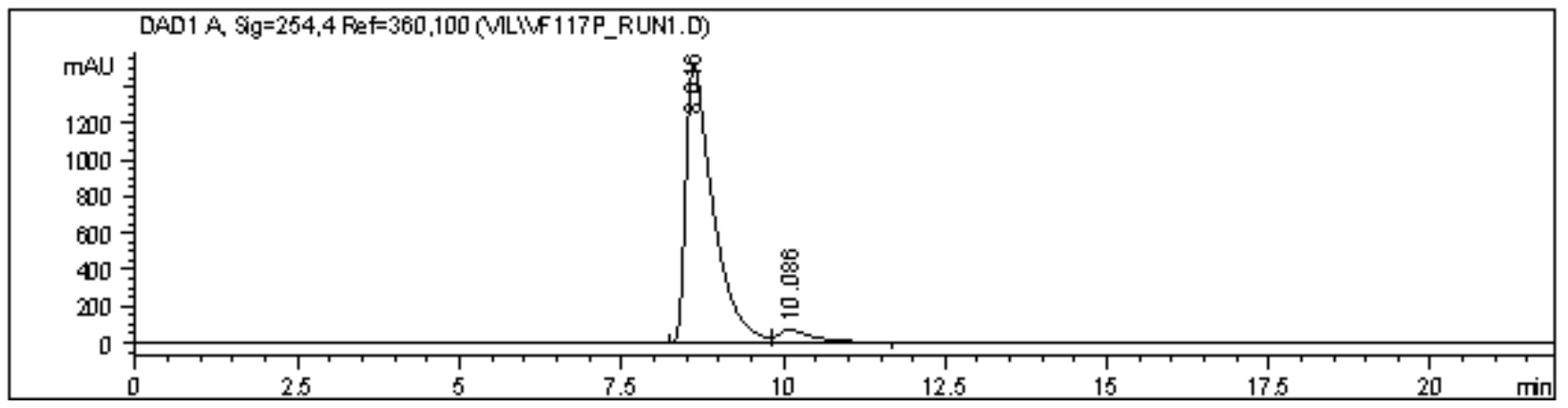

Signal 1: DADl A, Sigh 254,4 Ref $=360,100$

\begin{tabular}{|c|c|c|c|c|c|}
\hline $\begin{array}{c}\text { Peak } \\
\#\end{array}$ & $\begin{array}{l}\text { RetTime Type } \\
\text { [min] }\end{array}$ & $\begin{array}{l}\text { Width } \\
\text { [min] }\end{array}$ & $\begin{array}{c}\text { Area } \\
\text { [ma } \mathrm{m}^{*} \mathrm{~s} \text { ] }\end{array}$ & $\begin{array}{l}\text { Height } \\
\text { [miU] }\end{array}$ & $\begin{array}{c}\text { hrea } \\
\text { 믐 }\end{array}$ \\
\hline 1 & $8.616 \mathrm{BV}$ & 0.4152 & $4.41272 \mathrm{e} 4$ & 1517.52527 & 94.5946 \\
\hline 2 & $10.086 \mathrm{VB}$ & 0.4906 & 2521.57007 & 71.65304 & 5.4054 \\
\hline
\end{tabular}




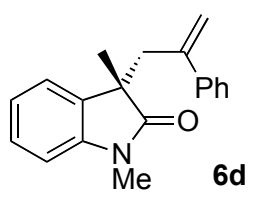

a) racemate

0J-R column, 95:5 Hex:IPA, $1 \mathrm{~mL} / \min , 30$ bar

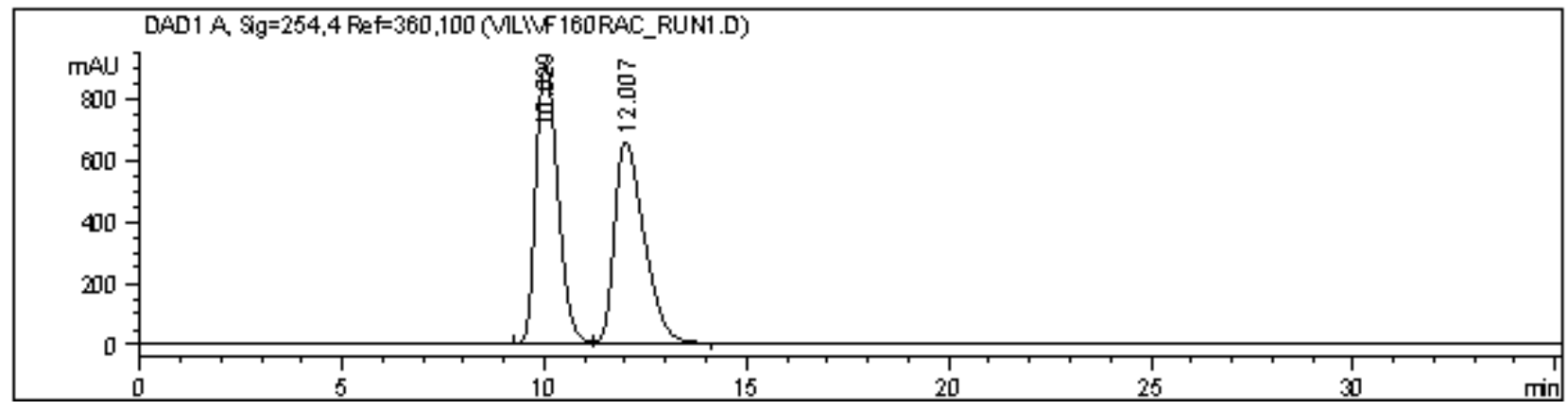

Signal 1: DADl A, Sig=254,4 Ref=360, 100

\begin{tabular}{|c|c|c|c|c|c|}
\hline $\begin{array}{c}\text { Peak } \\
\#\end{array}$ & $\begin{array}{l}\text { Ret Time Type } \\
\text { [min] }\end{array}$ & $\begin{array}{l}\text { Width } \\
\text { [min] }\end{array}$ & $\begin{array}{c}\text { Area } \\
{\left[\mathrm{maU}^{*} \mathrm{~s}\right]}\end{array}$ & $\begin{array}{l}\text { Height } \\
\text { [miU] }\end{array}$ & $\underset{\text { Area }}{\text { 믐 }}$ \\
\hline & & & & & \\
\hline 2 & $\begin{array}{l}10.029 \mathrm{BV} \\
12.007 \mathrm{VB}\end{array}$ & $\begin{array}{l}0.5982 \\
0.7749\end{array}$ & $\begin{array}{l}3.41167 \mathrm{e} 4 \\
3.31263 \mathrm{e} 4\end{array}$ & $\begin{array}{l}904.21796 \\
654.09021\end{array}$ & $\begin{array}{l}50.7365 \\
49.2635\end{array}$ \\
\hline
\end{tabular}

b) $88 \%$ ee

0J-R column, 95:5 Hex:IPA, $1.0 \mathrm{~mL} / \min , 30 \mathrm{bar}$

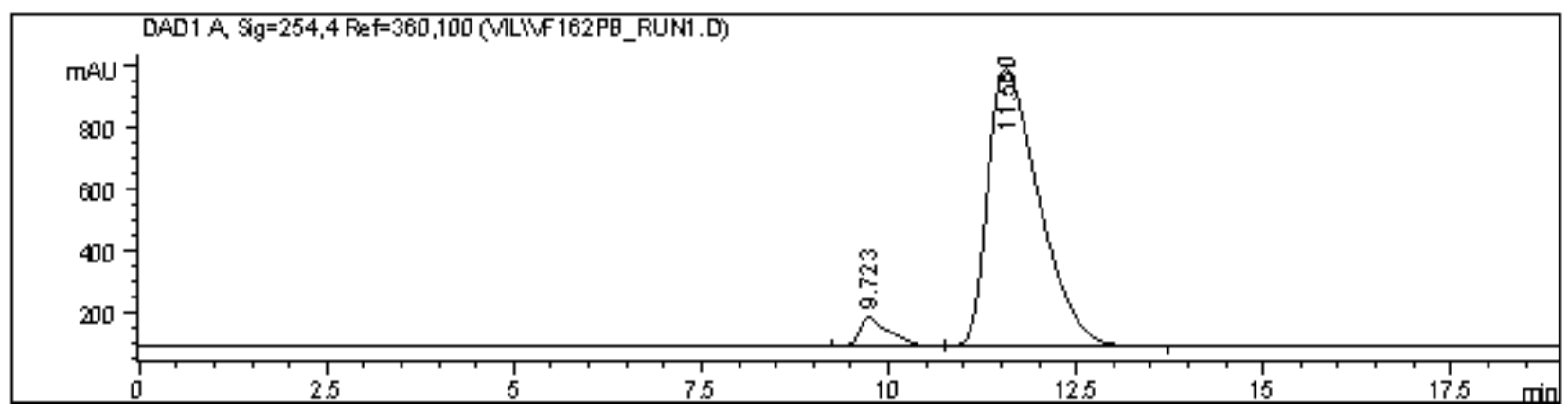

Signal 1: DADl A, Sig=254,4 Ref=360, 100

\begin{tabular}{|c|c|c|c|c|c|}
\hline $\begin{array}{c}\text { Peak } \\
\quad \#\end{array}$ & $\begin{array}{l}\text { Ret Time Type } \\
\text { [min] }\end{array}$ & $\begin{array}{l}\text { Width } \\
\text { [min] }\end{array}$ & $\begin{array}{c}\text { Area } \\
{\left[\min U^{*} s\right]}\end{array}$ & $\begin{array}{l}\text { Height } \\
\text { [miU] }\end{array}$ & $\begin{array}{c}\text { Area } \\
\text { 믑 }\end{array}$ \\
\hline & & &.---1 &.----1 & \\
\hline $\begin{array}{l}1 \\
2\end{array}$ & $\begin{array}{r}9.723 \mathrm{BV} \\
11.560 \mathrm{VB}\end{array}$ & & $\begin{array}{l}2618.04956 \\
4.26313 \mathrm{e} 4\end{array}$ & $\begin{array}{r}94.15912 \\
897.27618\end{array}$ & $\begin{array}{r}5.7858 \\
94.2142\end{array}$ \\
\hline
\end{tabular}




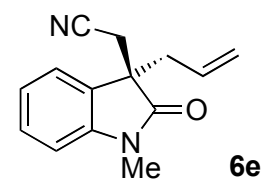

a) racemate

AD column, 98:2 Hex:IPA, $1.0 \mathrm{~mL} / \min , 24$ bar

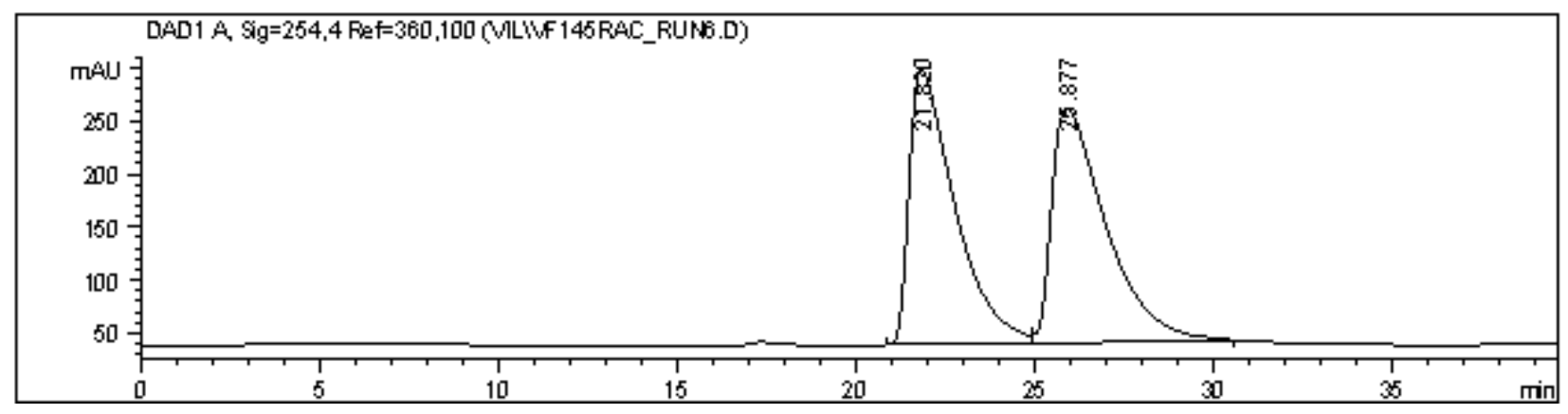

Signal 1: DADl A, Sig $=254,4$ Re $f=360,100$

\begin{tabular}{|c|c|c|c|c|c|}
\hline $\begin{array}{c}\text { Peak } \\
\#\end{array}$ & $\begin{array}{l}\text { Ret Time Type } \\
\text { [min] }\end{array}$ & $\begin{array}{l}\text { width } \\
\text { [min] }\end{array}$ & $\begin{array}{c}\text { Area } \\
\left.\text { [mind* } U^{*}\right]\end{array}$ & $\begin{array}{l}\text { Height } \\
\text { [miU] }\end{array}$ & $\begin{array}{c}\text { Area } \\
\text { 믑 }\end{array}$ \\
\hline 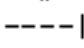 & & & ------- & $-0-1-0-0$ & \\
\hline 1 & $21.820 \mathrm{BV}$ & 1. 2842 & 2. $30154 \mathrm{e} 4$ & 260.14398 & 49.4511 \\
\hline 2 & $25.877 \mathrm{VB}$ & 1. 5679 & $2.35263 \mathrm{e} 4$ & 222.44762 & 50.5489 \\
\hline
\end{tabular}

b) $68 \%$ ee

AD column, 98:2 Hex: IPA, $1.0 \mathrm{~mL} / \min , 24$ bar

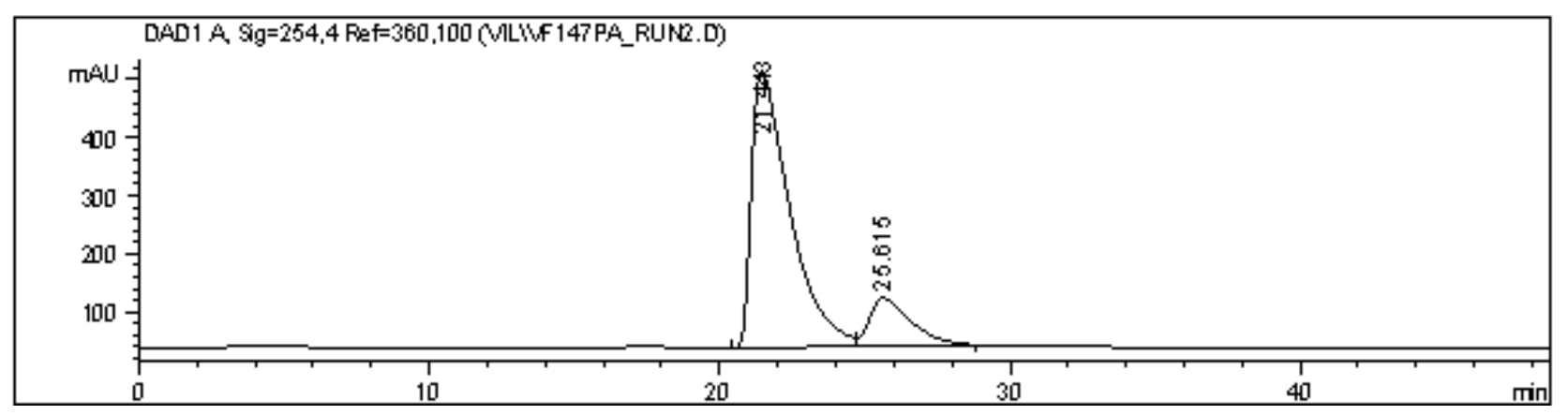

Signal 1: DADl A, Sig $=254,4$ Ref $=360,100$

\begin{tabular}{|c|c|c|c|c|c|}
\hline $\begin{array}{c}\text { Peak } \\
\#\end{array}$ & $\begin{array}{l}\text { RetTime Type } \\
\text { [min] }\end{array}$ & $\begin{array}{l}\text { Width } \\
\text { [min] }\end{array}$ & $\begin{array}{c}\text { Area } \\
\text { [mit*s] }\end{array}$ & $\begin{array}{l}\text { Height } \\
\text { [miU] }\end{array}$ & $\begin{array}{c}\text { Area } \\
\text { 몹 }\end{array}$ \\
\hline & & ----- & ---------- & --------ー- & -------- \\
\hline 1 & $21.443 \mathrm{BV}$ & 1. 3464 & $4.37320 \mathrm{e} 4$ & 474.89600 & 1329 \\
\hline 2 & $25.615 \mathrm{VB}$ & 1. 4035 & 8247.66016 & 82.56102 & 8671 \\
\hline
\end{tabular}




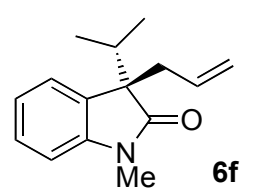

a) racemate

AD column, 97:3 Hex: (9: 1 Hex:IPA), 0.5 mL/min, 12 bar

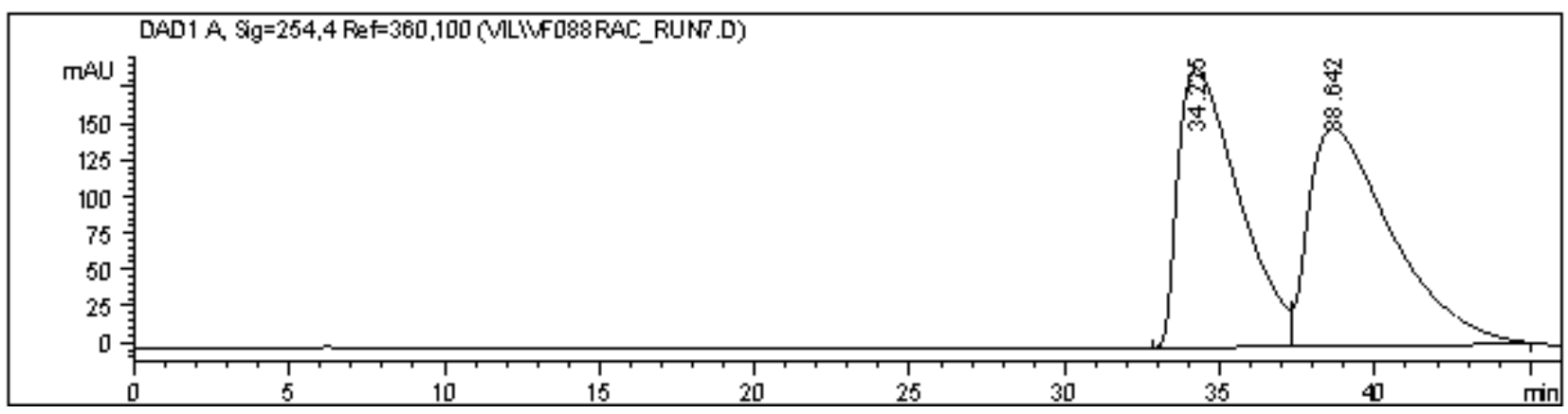

Signal 1: DADl A, Sig $=254,4$ Ref $=360,100$

\begin{tabular}{|c|c|c|c|c|c|}
\hline $\begin{array}{c}\text { Peaz } \\
\ldots\end{array}$ & $\begin{array}{l}\text { Ret Time Type } \\
\text { [min] }\end{array}$ & $\begin{array}{l}\text { Width } \\
\text { [min] }\end{array}$ & $\begin{array}{c}\text { Area } \\
{\left[\mathrm{mid}^{*} \mathrm{~s}\right]}\end{array}$ & $\begin{array}{l}\text { Height } \\
\text { [miU] }\end{array}$ & $\begin{array}{c}\text { Area } \\
\text { 몹 }\end{array}$ \\
\hline & & & & & \\
\hline $\begin{array}{l}1 \\
2\end{array}$ & $\begin{array}{l}34.225 \mathrm{BV} \\
38.642 \mathrm{VB}\end{array}$ & & $\begin{array}{l}2.59314 \mathrm{e} 4 \\
2.80061 \mathrm{e} 4\end{array}$ & $\begin{array}{l}190.74402 \\
149.21870\end{array}$ & \\
\hline
\end{tabular}

b) $72 \%$ ee

AD column, 97:3 Hex: (9:1 Hex:IPA), $0.5 \mathrm{~mL} / \min , 11$ bar

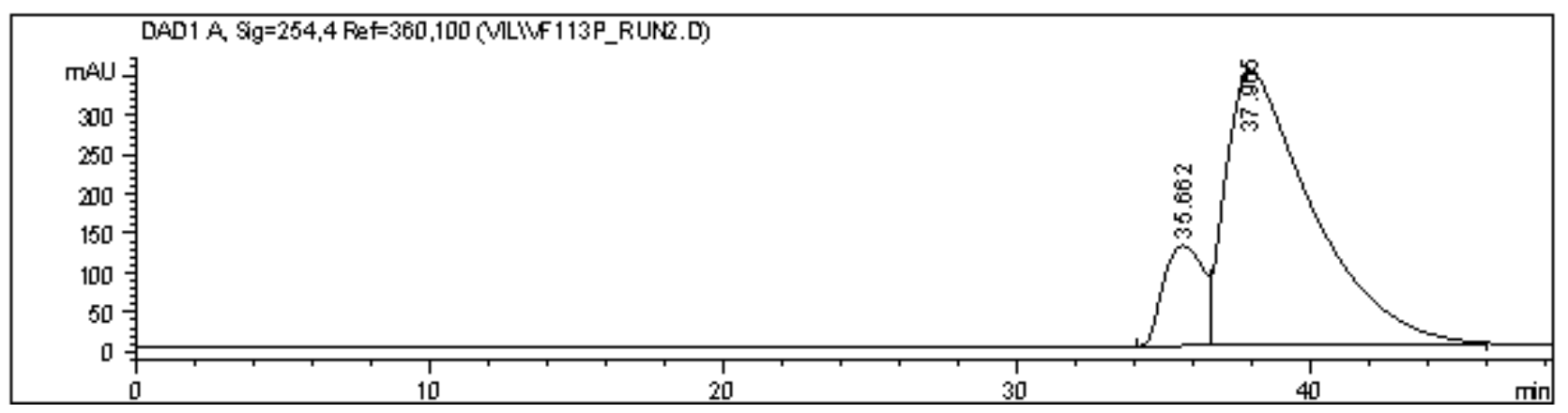

Signal 1: DADl A, Sig $=254,4$ Ref $=360,100$

\begin{tabular}{|c|c|c|c|c|c|}
\hline $\begin{array}{c}\mathrm{Peal} \\
\#\end{array}$ & $\begin{array}{l}\text { Ret Time Type } \\
\text { [min] }\end{array}$ & $\begin{array}{l}\text { Width } \\
\text { [min] }\end{array}$ & 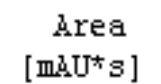 & $\begin{array}{l}\text { Height } \\
\text { [miU] }\end{array}$ & $\begin{array}{c}\text { Area } \\
\text { 믑 }\end{array}$ \\
\hline 1 & $35.662 \mathrm{BV}$ & 1. 5459 & 1. $18417 \mathrm{e} 4$ & 126.52760 & 14.2537 \\
\hline 2 & $37.965 \mathrm{VB}$ & 2.8813 & 7. $12363 \mathrm{e} 4$ & 348.71661 & 85.7463 \\
\hline
\end{tabular}




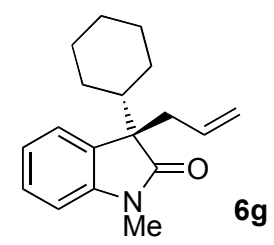

a) racemate

0J-R column, 99:1 Hex:IPA, $0.5 \mathrm{~mL} / \min , 14 \mathrm{bar}$

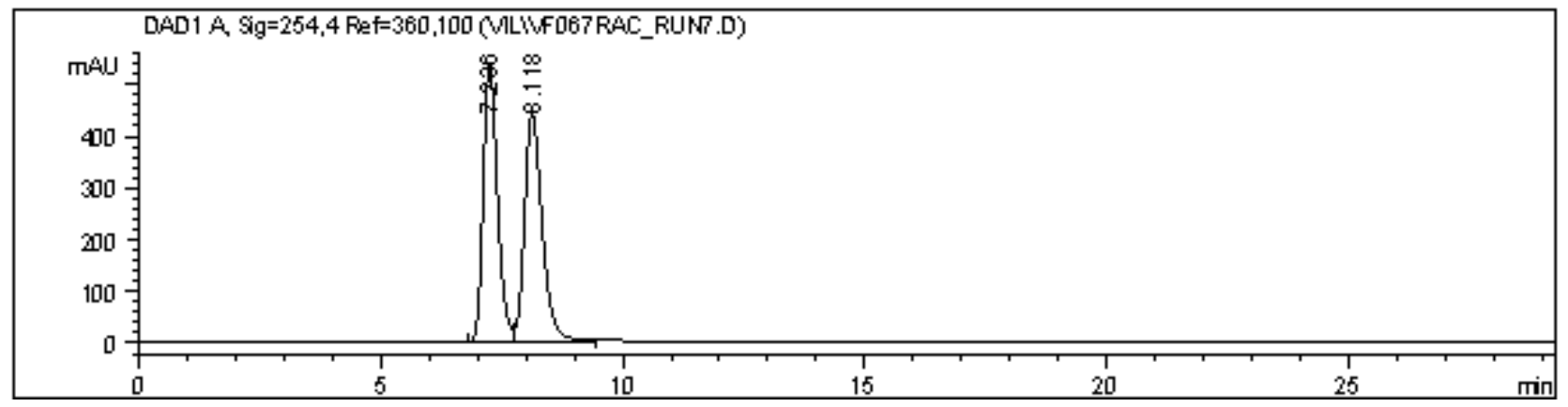

Signal 1: DADl A, Sig $=254,4$ Ref $=360,100$

\begin{tabular}{|c|c|c|c|c|c|}
\hline $\begin{array}{c}\text { Peak } \\
\#\end{array}$ & $\begin{array}{l}\text { RetTime Type } \\
\text { [min] }\end{array}$ & $\begin{array}{l}\text { Width } \\
\text { [min] }\end{array}$ & $\begin{array}{c}\text { Area } \\
{\left[\mathrm{ma}^{2} \mathrm{U}^{*} \mathrm{~s}\right]}\end{array}$ & $\begin{array}{l}\text { Height } \\
\text { [miU] }\end{array}$ & $\begin{array}{c}\text { hrea } \\
\text { 몹 }\end{array}$ \\
\hline 1 & $7236 \mathrm{BU}$ & & & 535,46173 & \\
\hline 2 & $8.118 \mathrm{VV}$ & 0.3857 & 1. $12182 \mathrm{e} 4$ & 442.57620 & 50.7095 \\
\hline
\end{tabular}

b) $76 \%$ ee

0J-R column, 99:1 Hex:IPA, $0.5 \mathrm{~mL} / \min , 14 \mathrm{bar}$

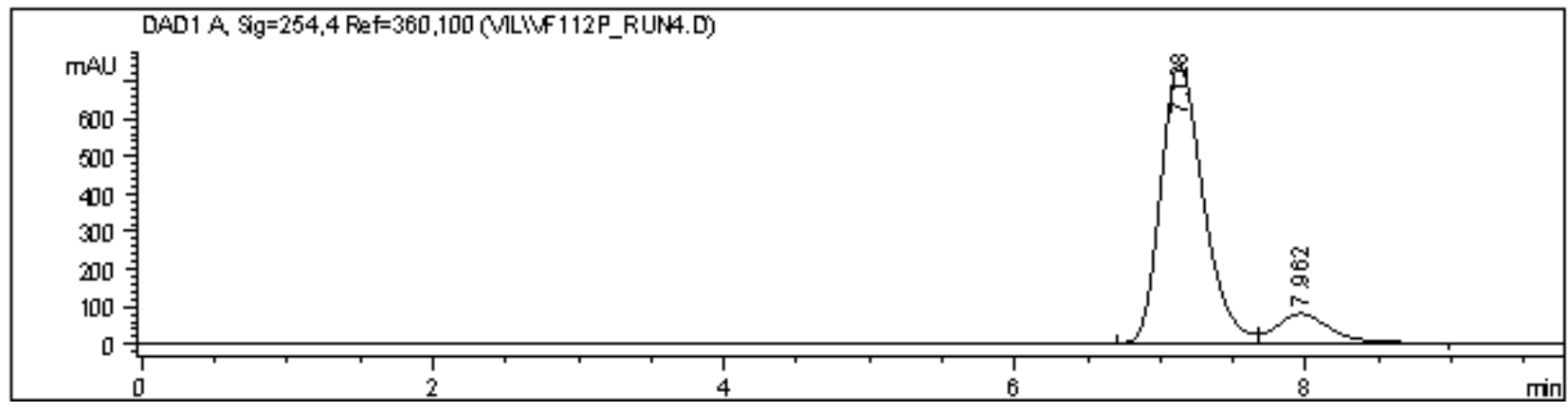

Signal 1: DADl A, Sig=254,4 Ref=360, 100

\begin{tabular}{|c|c|c|c|c|c|}
\hline $\begin{array}{c}\text { Peak } \\
\#\end{array}$ & $\begin{array}{l}\text { Ret Time Type } \\
\text { [min] }\end{array}$ & $\begin{array}{l}\text { Width } \\
\text { [min] }\end{array}$ & $\begin{array}{c}\text { Area } \\
{\left[\mathrm{mLU}^{\star} \mathrm{s}\right]}\end{array}$ & $\begin{array}{l}\text { Height } \\
\text { [miU] }\end{array}$ & $\begin{array}{c}\text { Area } \\
\text { 믑 }\end{array}$ \\
\hline & 7.12 & & .50 & 15613 & 516 \\
\hline 2 & $7.962 \mathrm{VB}$ & 0.3911 & 2046.10754 & 78.24133 & 11.9484 \\
\hline
\end{tabular}




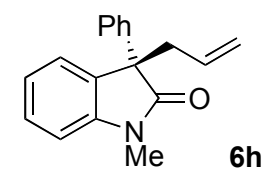

a) racemate

0J-R column, 90:10 Hex: IPA, $1 \mathrm{~mL} / \mathrm{min}, 30 \mathrm{bar}$

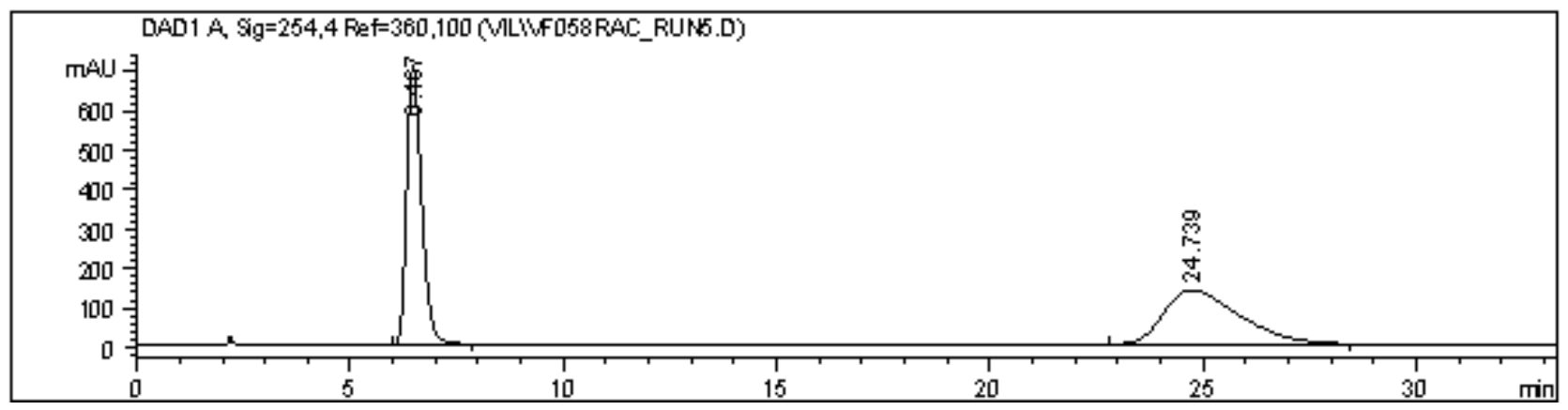

Signal 1: DADl A, Sig=254,4 Ref=360, 100

\begin{tabular}{|c|c|c|c|c|c|}
\hline $\begin{array}{c}\text { Pealz } \\
\#\end{array}$ & $\begin{array}{l}\text { Ret Time Type } \\
\text { [min] }\end{array}$ & $\begin{array}{l}\text { Width } \\
\text { [min] }\end{array}$ & 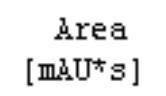 & $\begin{array}{l}\text { Height } \\
\text { [miU] }\end{array}$ & $\underset{\substack{\text { Area } \\
\text { 믐 }}}{ }$ \\
\hline & & & & & \\
\hline 2 & $24.739 \mathrm{BB}$ & 1.8878 & 1. $70367 \mathrm{e} 4$ & 137.47829 & 49.7478 \\
\hline
\end{tabular}

b) $79 \%$ ee

0J-R column, 90:10 Hex:IPA, $1.0 \mathrm{~mL} / \min , 31$ bar

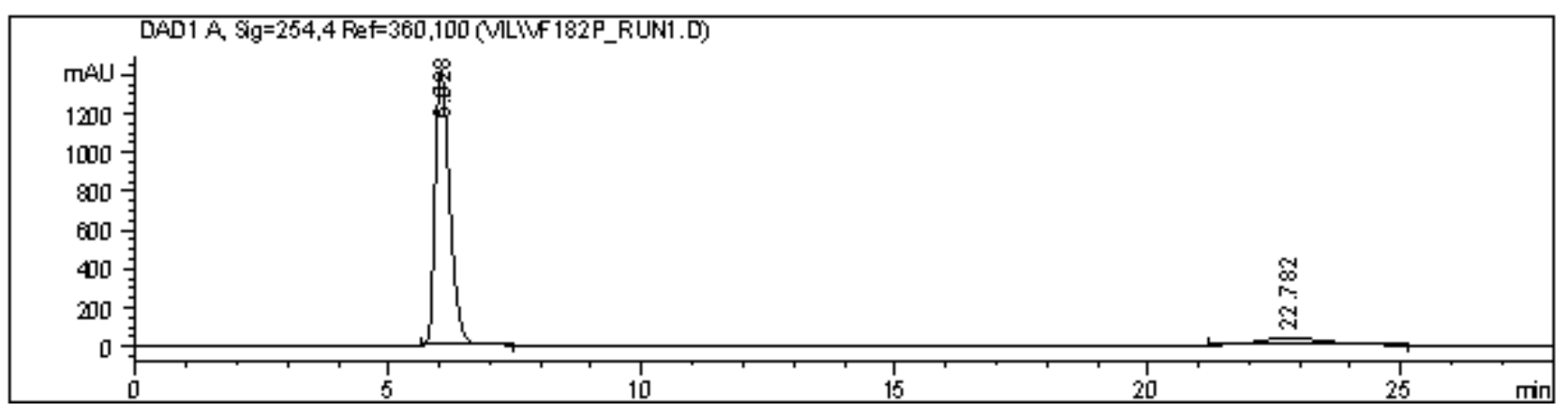

Signal 1: DADl A, Sig $=254,4$ Ref $=360,100$

\begin{tabular}{|c|c|c|c|c|c|}
\hline $\begin{array}{c}\text { Pealz } \\
\#\end{array}$ & $\begin{array}{l}\text { RetTime Type } \\
\text { [min] }\end{array}$ & $\begin{array}{l}\text { Width } \\
\text { [min] }\end{array}$ & $\begin{array}{c}\text { Area } \\
{\left[\mathrm{mid}^{*} \mathrm{~s}\right]}\end{array}$ & $\begin{array}{l}\text { Height } \\
\text { [miU] }\end{array}$ & $\begin{array}{c}\text { Area } \\
\text { 몸 }\end{array}$ \\
\hline 1 & 6.0281 & 309 & $2.84164 \mathrm{e} 4$ & 1417.23218 & 89.6468 \\
\hline 2 & $22.782 \mathrm{BB}$ & 1. 3262 & 3281.78491 & 36.11427 & 10.3532 \\
\hline
\end{tabular}




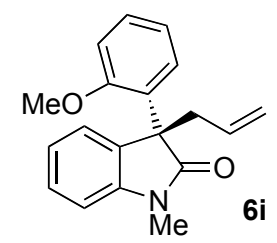

a) racemate

0J-R column, 90:10 Hex: IPA, $1.0 \mathrm{~mL} / \mathrm{min}, 31 \mathrm{bar}$

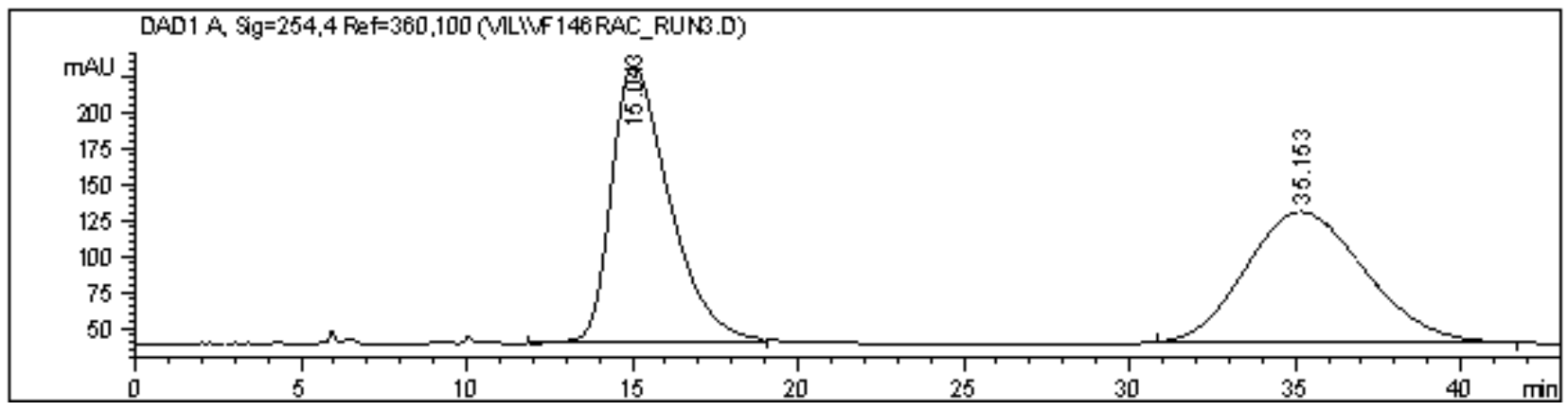

Signal 1: DADl A, Sig $=254,4$ Ref $=360,100$

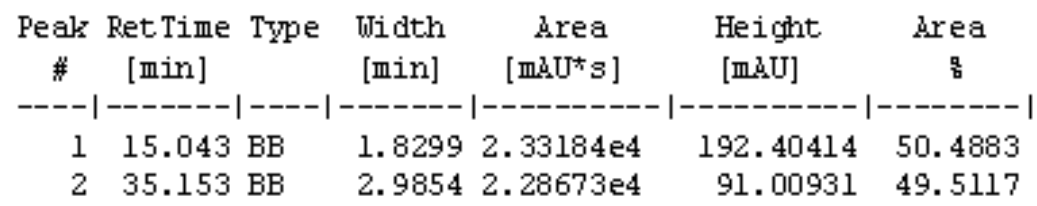

b) $95 \%$ ee

0J-R column, 90:10 Hex: IPA, $1.0 \mathrm{~mL} / \mathrm{min}, 31 \mathrm{bar}$

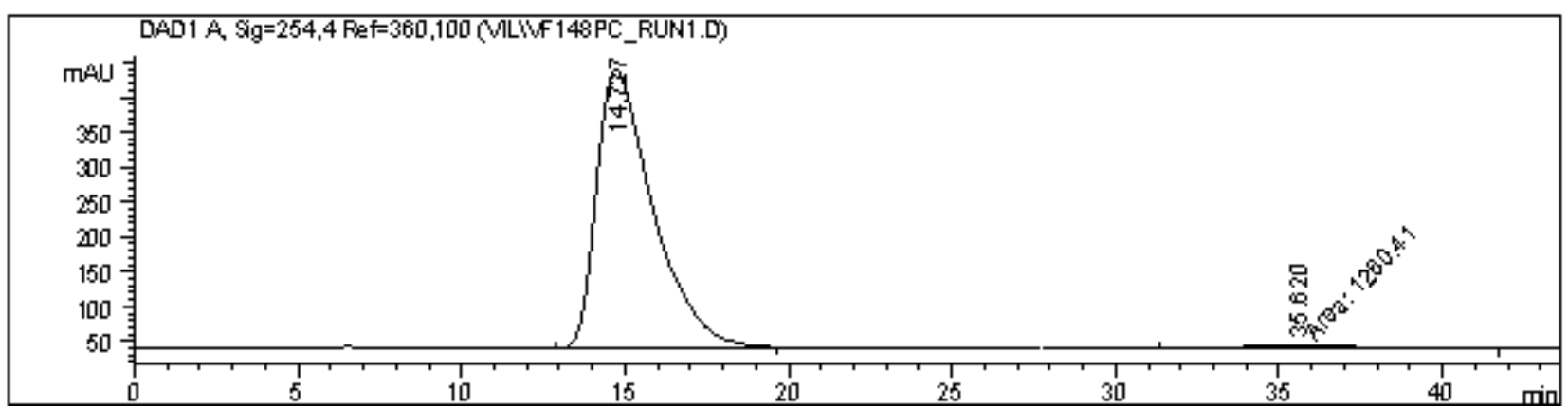

Signal 1: DADl A, Sig $=254,4$ Ref $=360,100$

\begin{tabular}{|c|c|c|c|c|c|}
\hline $\begin{array}{c}\text { Pealz } \\
\#\end{array}$ & $\begin{array}{l}\text { RetTime Type } \\
\text { [min] }\end{array}$ & $\begin{array}{l}\text { Width } \\
\text { [min] }\end{array}$ & $\begin{array}{c}\text { Area } \\
{\left[\mathrm{ma}^{*} \mathrm{U} \mathrm{N}^{*} \mathrm{~s}\right.}\end{array}$ & $\begin{array}{l}\text { Height } \\
\text { [miU] }\end{array}$ & $\begin{array}{c}\text { Area } \\
\text { 믐 }\end{array}$ \\
\hline & תחים תחת & & 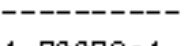 & & \\
\hline $\begin{array}{l}1 \\
2\end{array}$ & $\begin{array}{l}14.727 \mathrm{BB} \\
35.620 \mathrm{MM}\end{array}$ & & $\begin{array}{l}4.79978 \mathrm{e} 4 \\
1260.41345\end{array}$ & $\begin{array}{r}398.80563 \\
5.45893\end{array}$ & $\begin{array}{r}97.4412 \\
2.5588\end{array}$ \\
\hline
\end{tabular}




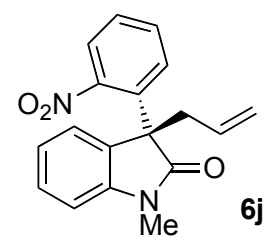

a) racemate

0J-R column, 90:10 Hex: IPA, $1.0 \mathrm{~mL} / \min , 30 \mathrm{bar}$

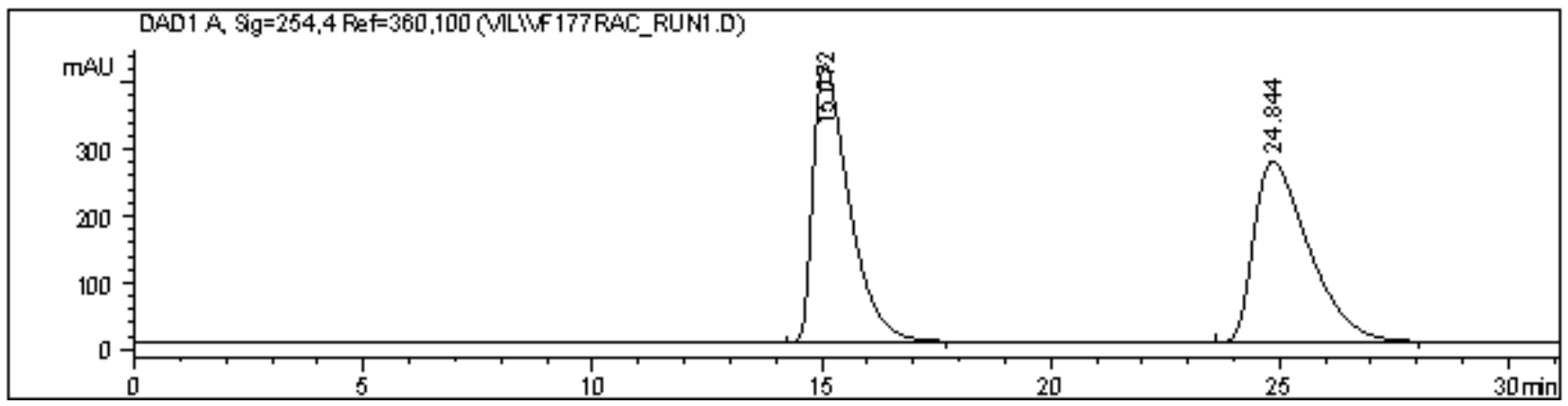

Signal 1: DADl A, Sig $=254,4$ Ref $=360,100$

\begin{tabular}{|c|c|c|c|c|c|}
\hline $\begin{array}{c}\text { Peak } \\
\ldots\end{array}$ & $\begin{array}{l}\text { Ret Time Type } \\
\text { [min] }\end{array}$ & $\begin{array}{l}\text { Width } \\
\text { [min] }\end{array}$ & $\begin{array}{c}\text { Area } \\
{\left[\mathrm{m}^{2} \mathrm{U}^{*} \mathrm{~s}\right]}\end{array}$ & $\begin{array}{l}\text { Height } \\
\text { [miU] }\end{array}$ & 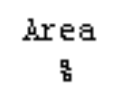 \\
\hline & & & & & \\
\hline 2 & $24.844 \mathrm{BB}$ & & $2.28356 \mathrm{e} 4$ & 271.54285 & 49.9777 \\
\hline
\end{tabular}

b) $87 \%$ ee

0J-R column, 90:10 Hex: IPA, $1.0 \mathrm{~mL} / \min , 31$ bar

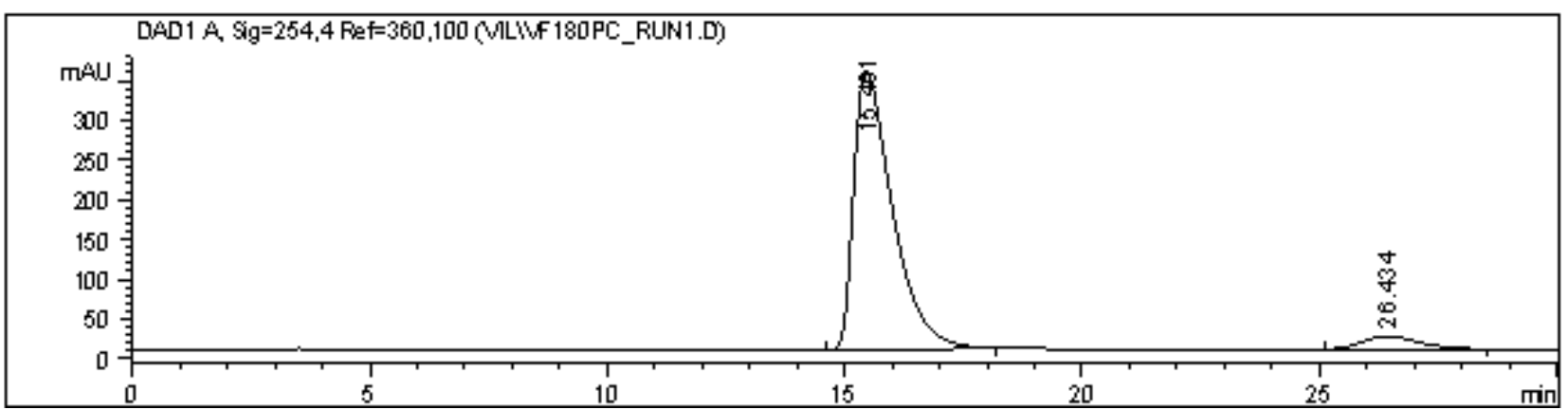

Signal 1: DADl A, Sig=254,4 Ref=360, 100

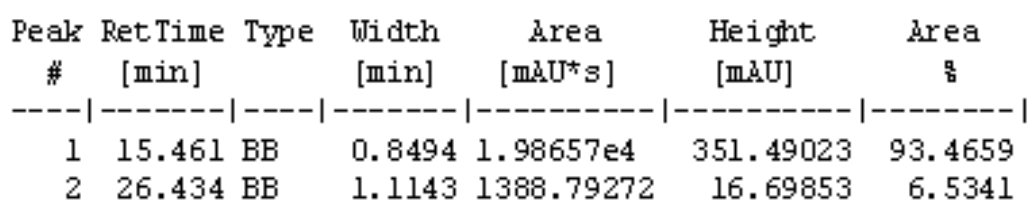




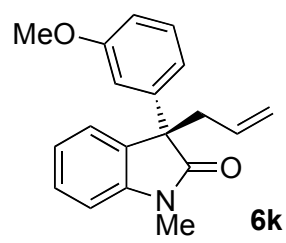

a) racemate

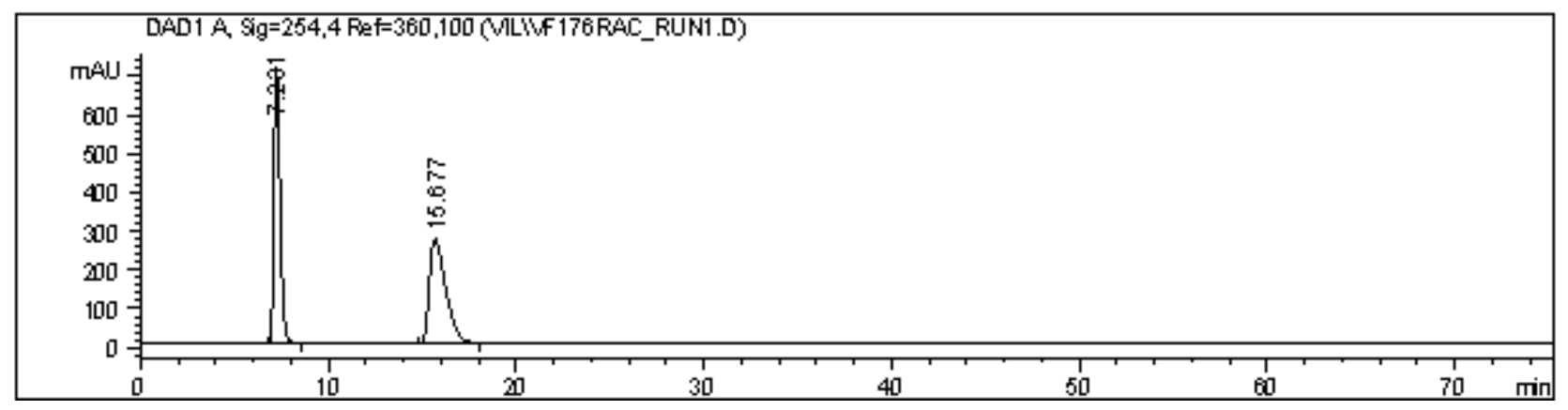

Signal 1: DADl A, Sig $=254,4$ Ref $=360,100$

\begin{tabular}{|c|c|c|c|c|c|}
\hline $\begin{array}{c}\text { Peak } \\
\ldots\end{array}$ & $\begin{array}{l}\text { RetTime Type } \\
\text { [min] }\end{array}$ & $\begin{array}{l}\text { Width } \\
\text { [min] }\end{array}$ & $\begin{array}{c}\text { Area } \\
\left.\text { [ma } \mathrm{m}^{*} \mathrm{~s}\right]\end{array}$ & $\begin{array}{l}\text { Height } \\
\text { [miU] }\end{array}$ & $\begin{array}{c}\text { Area } \\
\text { 믑 }\end{array}$ \\
\hline & & - & -- & 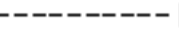 & ------- \\
\hline 1 & 7.23 & 0.3 & 1. $64583 \mathrm{e} 4$ & & \\
\hline 2 & 15 . & & $1.64656 \mathrm{e} 4$ & 270.59 & 50. \\
\hline
\end{tabular}

b) $78 \%$ ee

0J-R column, 90:10 Hex:IPA, $1.0 \mathrm{~mL} / \min , 31$ bar

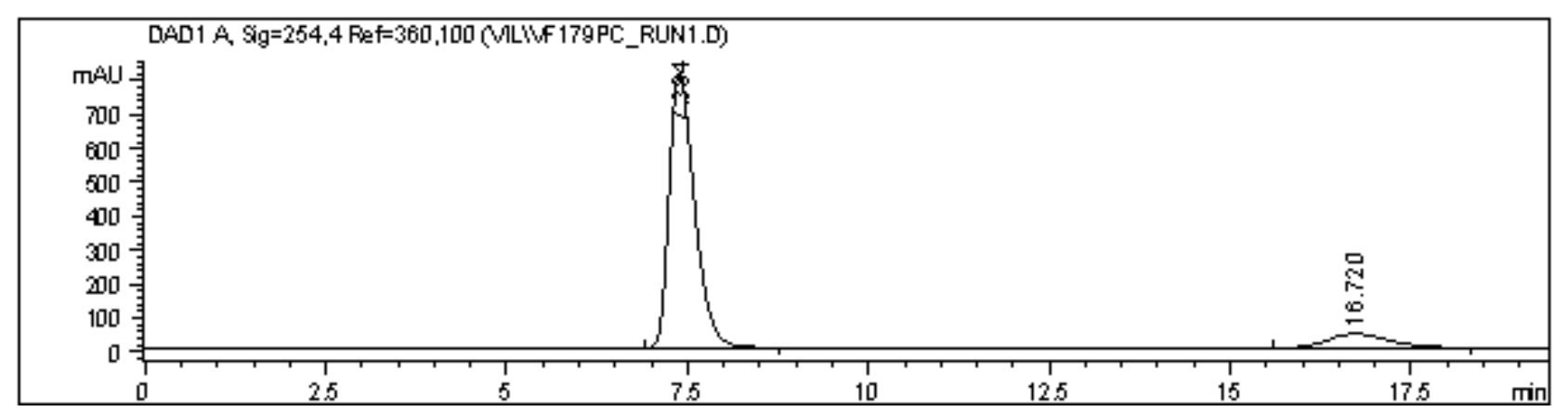

Signal 1: DADl A, sig $=254,4$ Ref $=360,100$

\begin{tabular}{|c|c|c|c|c|c|}
\hline $\begin{array}{c}\text { Peak } \\
\#\end{array}$ & $\begin{array}{l}\text { Ret Time Type } \\
\text { [min] }\end{array}$ & $\begin{array}{l}\text { Width } \\
\text { [min] }\end{array}$ & $\begin{array}{c}\text { Area } \\
\text { [ma } \mathrm{mU}^{*} \mathrm{~s} \text { ] }\end{array}$ & $\begin{array}{l}\text { Height } \\
\text { [miU] }\end{array}$ & $\begin{array}{l}\text { Area } \\
\text { 믐 }\end{array}$ \\
\hline 1 & $7.384 \mathrm{~B}$ & 0.37 & 1.983 & 811. & 3876 \\
\hline 2 & $16.720 \mathrm{BB}$ & 0.9226 & 2479.63794 & 41.29181 & 11.1124 \\
\hline
\end{tabular}


7. ${ }^{1} \mathrm{H}$ NMR Spectra

$\mathrm{CDCl}_{3}, 400 \mathrm{MHz}$

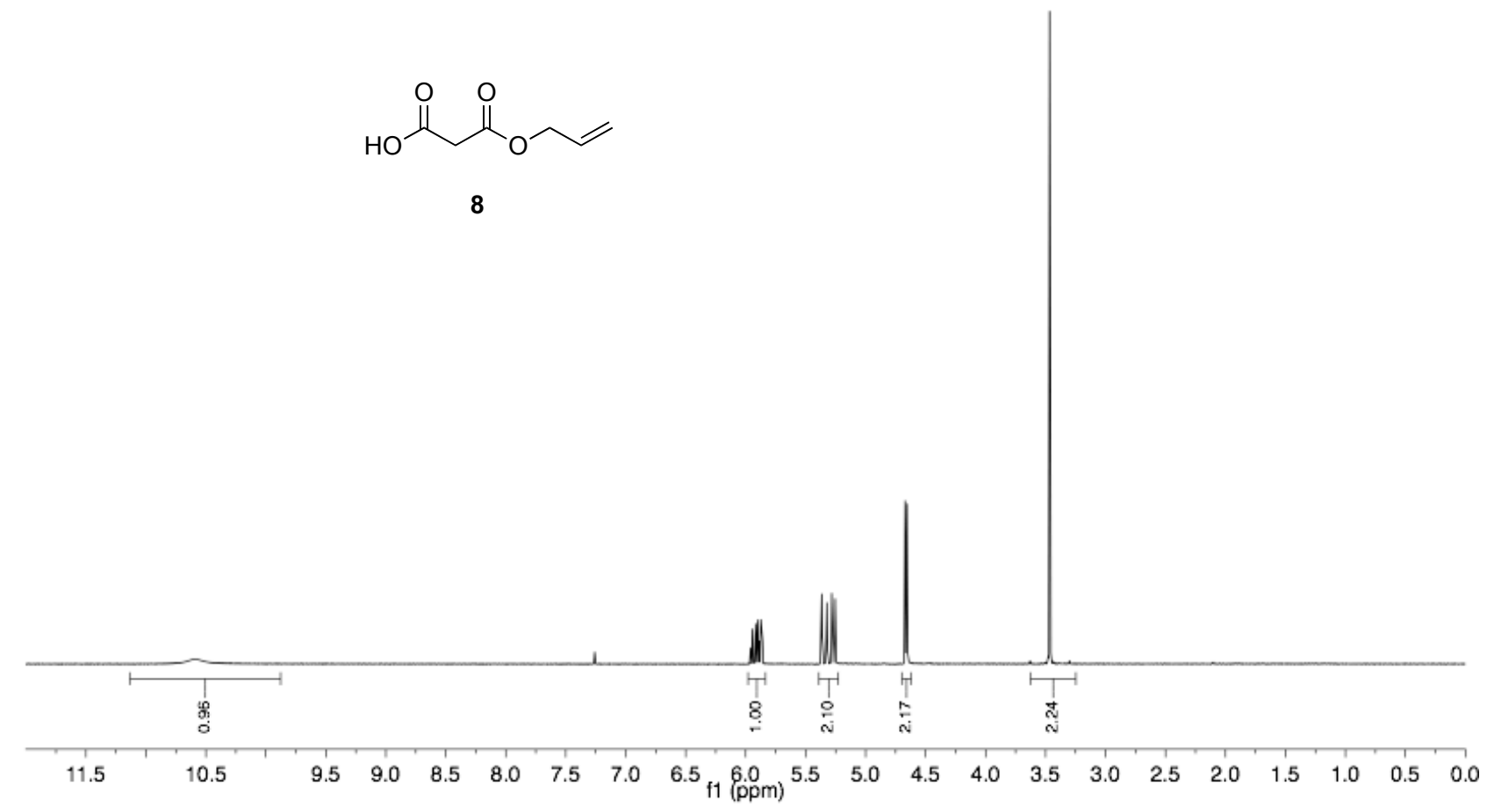

$\mathrm{CDCl}_{3}, 400 \mathrm{MHz}$<smiles>C=C(C)COC(=O)CC(=O)O</smiles>

9

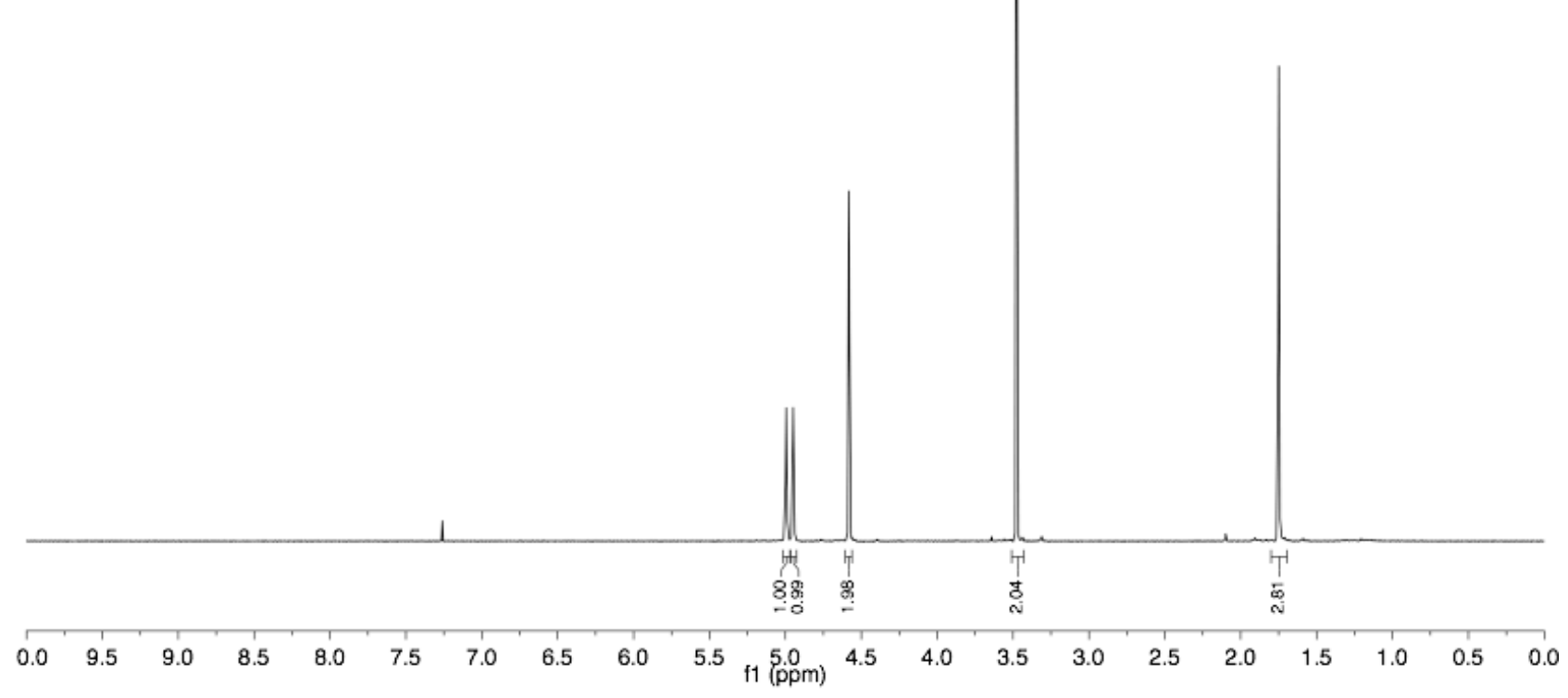


$\mathrm{CDCl}_{3}, 400 \mathrm{MHz}$

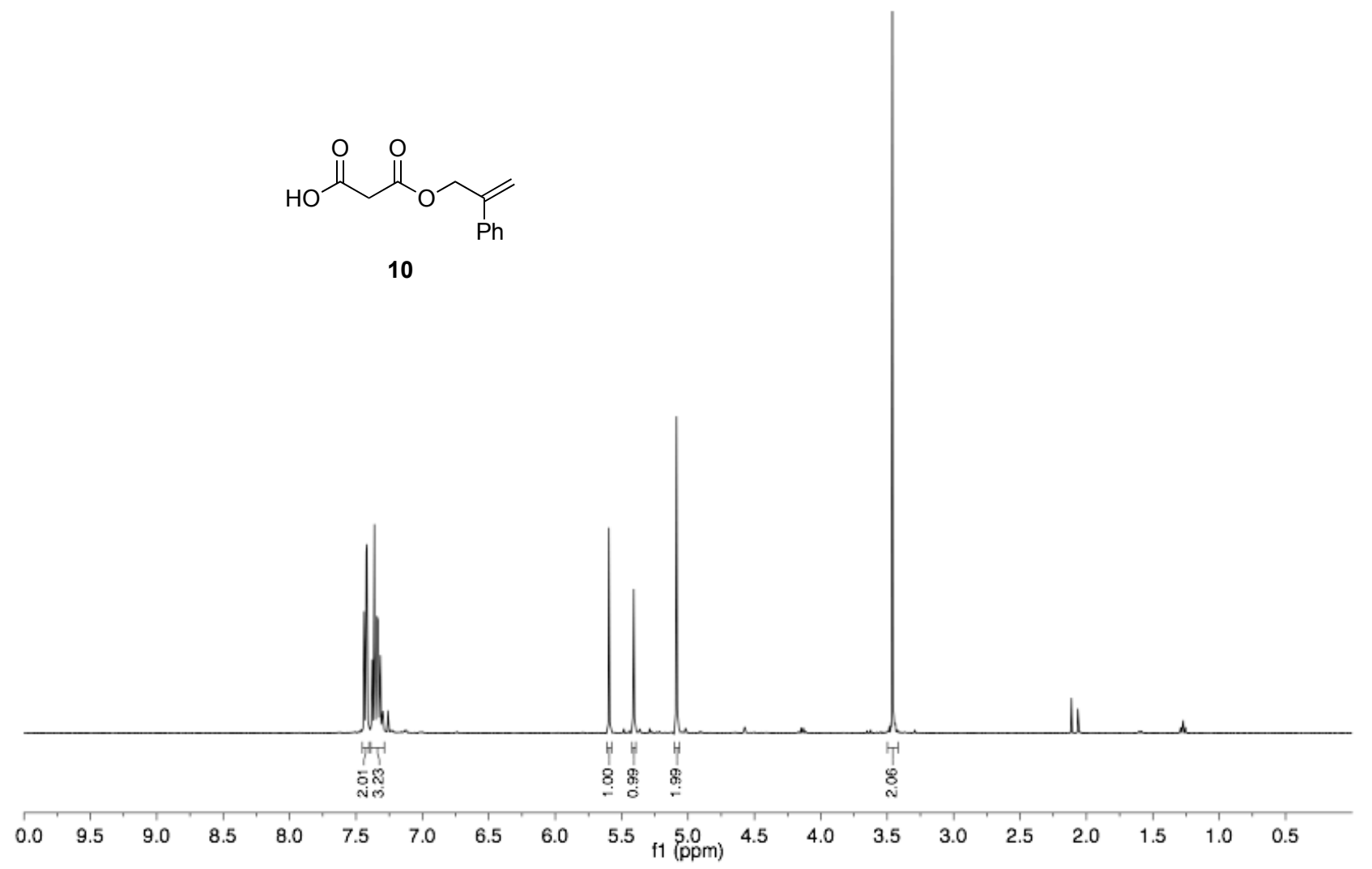

$\mathrm{CDCl}_{3}, 400 \mathrm{MHz}$

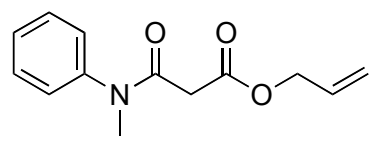

12

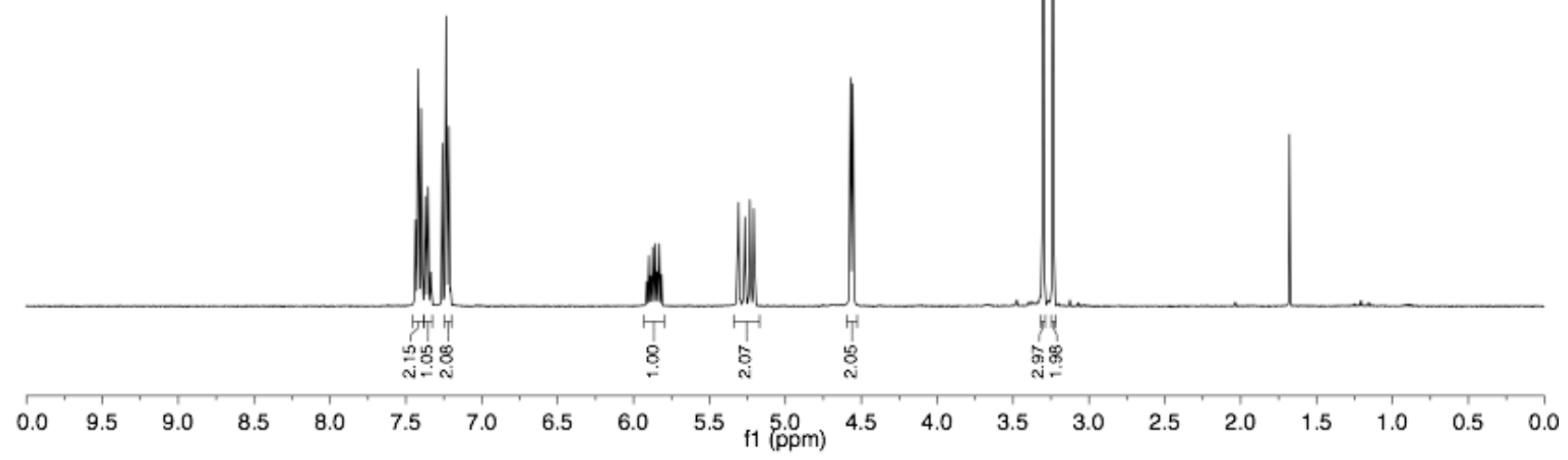


$\mathrm{CDCl}_{3}, 400 \mathrm{MHz}$

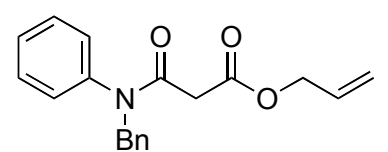

13

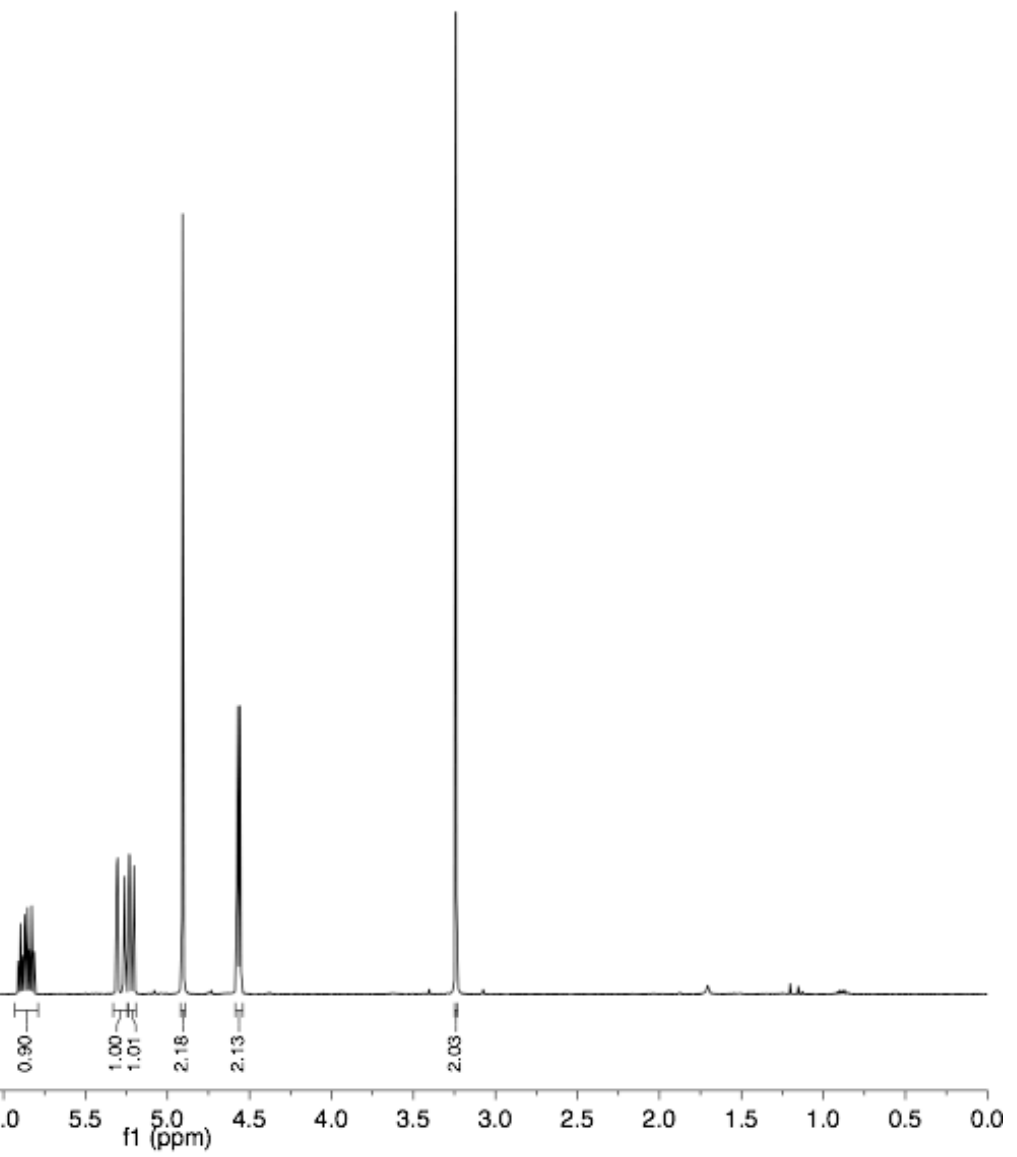

$\mathrm{CDCl}_{3}, 400 \mathrm{MHz}$
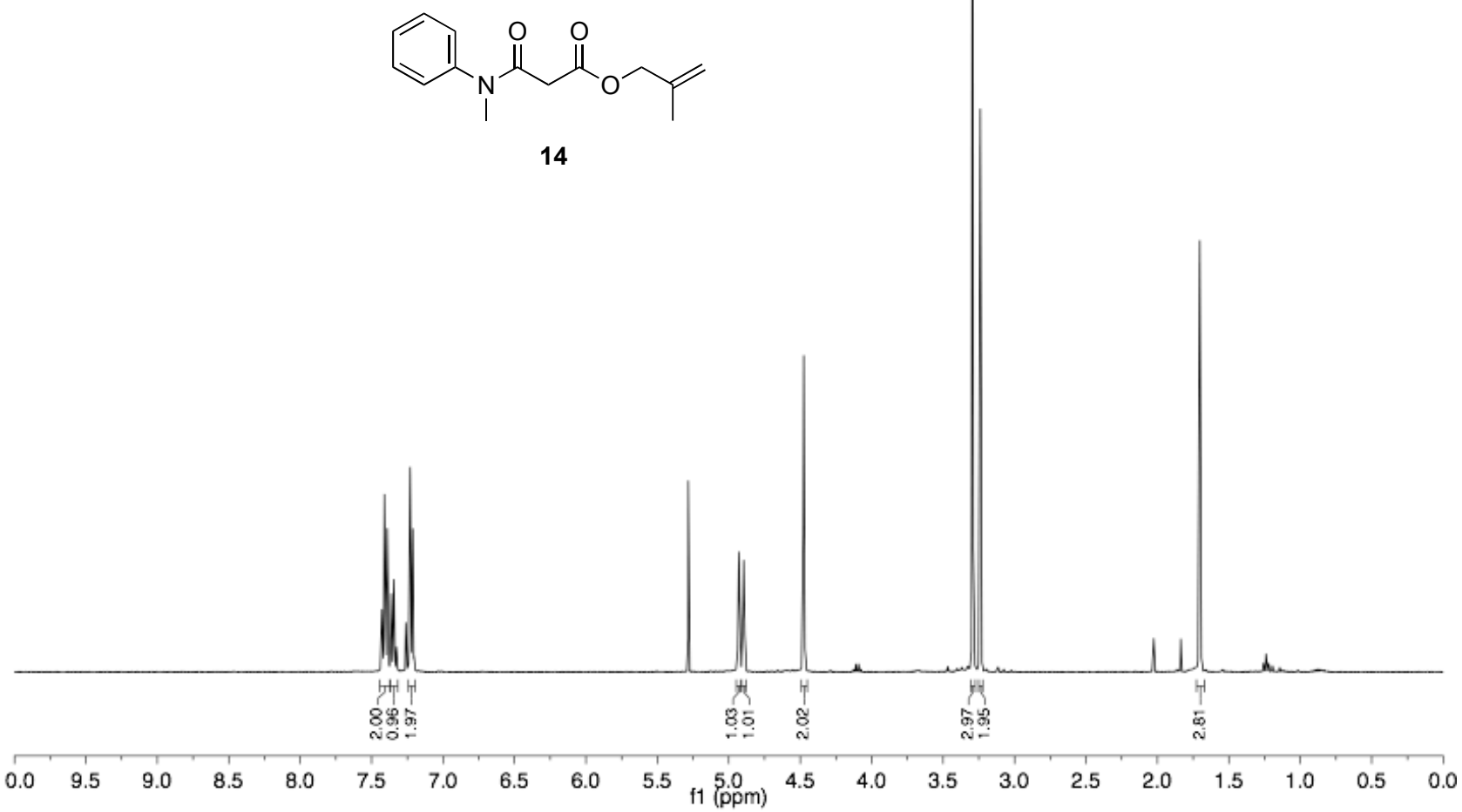
$\mathrm{CDCl}_{3}, 400 \mathrm{MHz}$

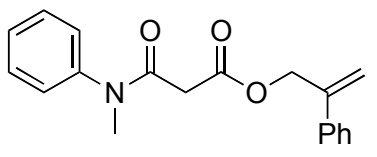

15

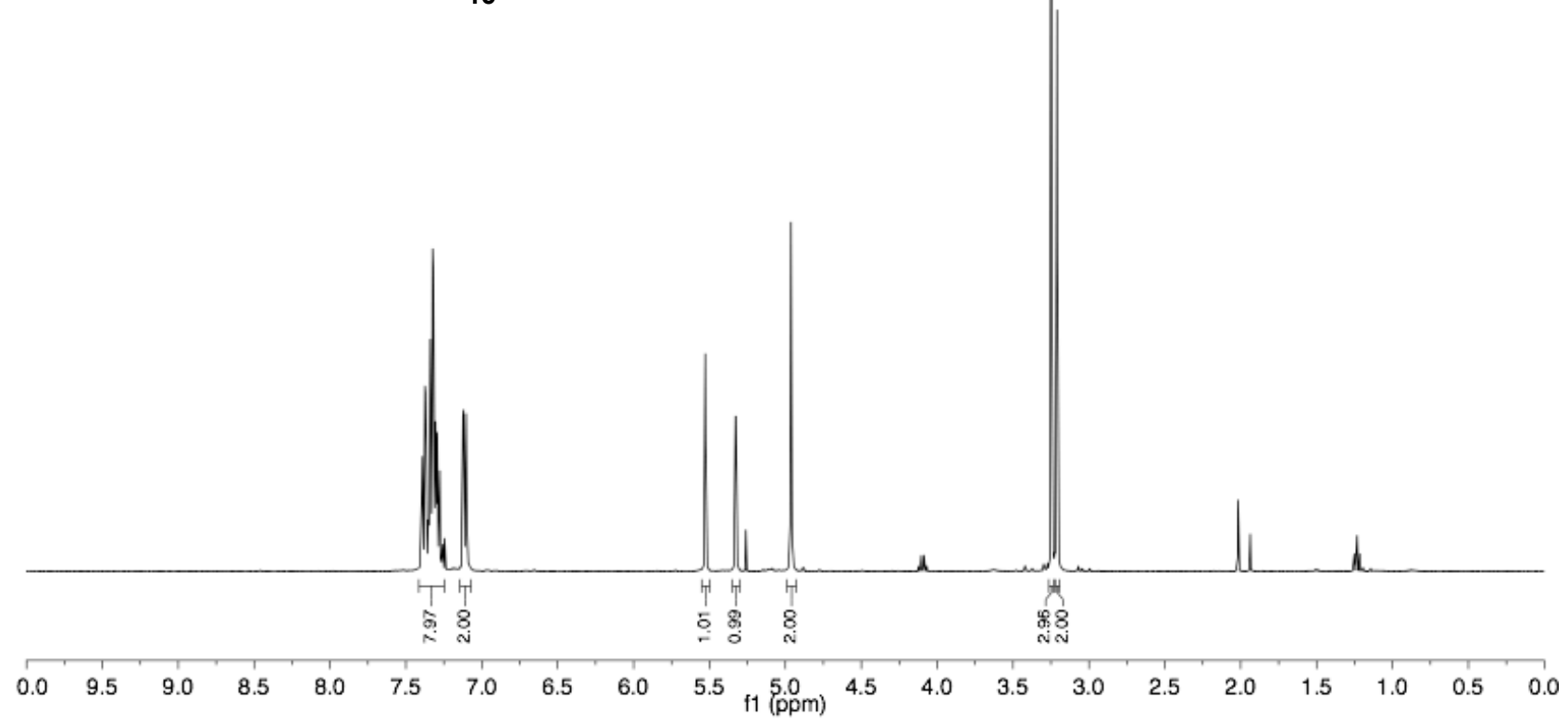

$\mathrm{CDCl}_{3}, 400 \mathrm{MHz}$

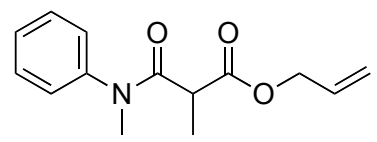

16

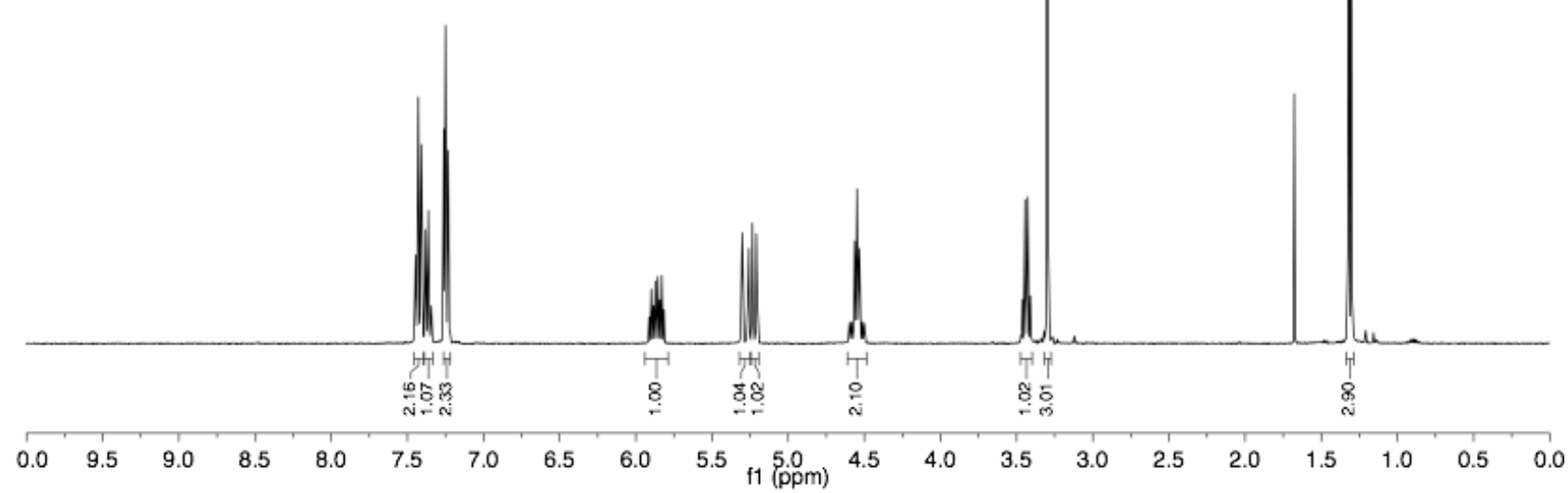


$\mathrm{CDCl}_{3}, 400 \mathrm{MHz}$
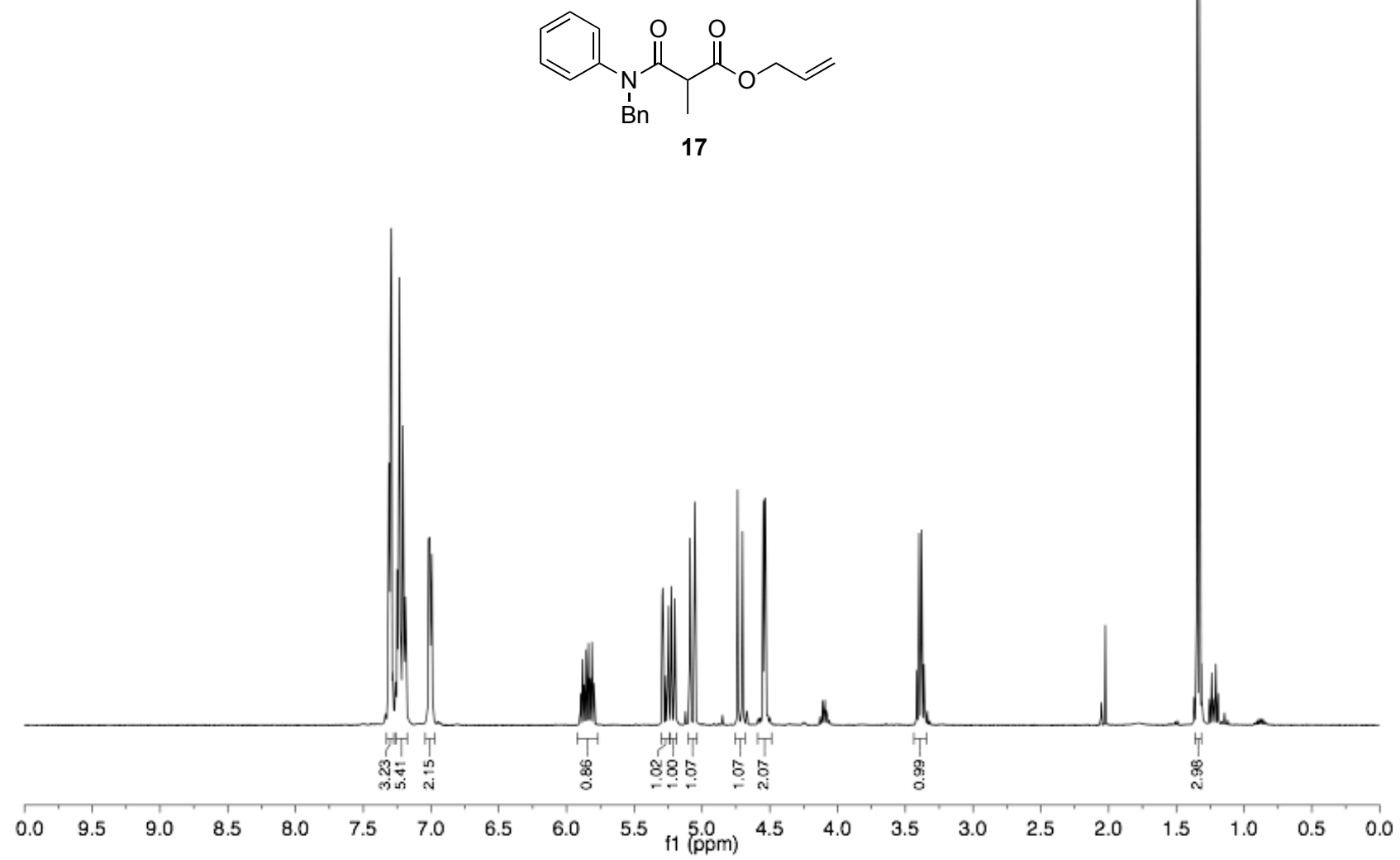

$\mathrm{CDCl}_{3}, 400 \mathrm{MHz}$

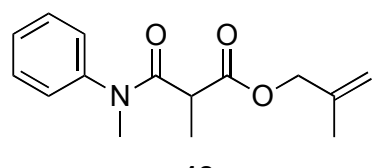

18

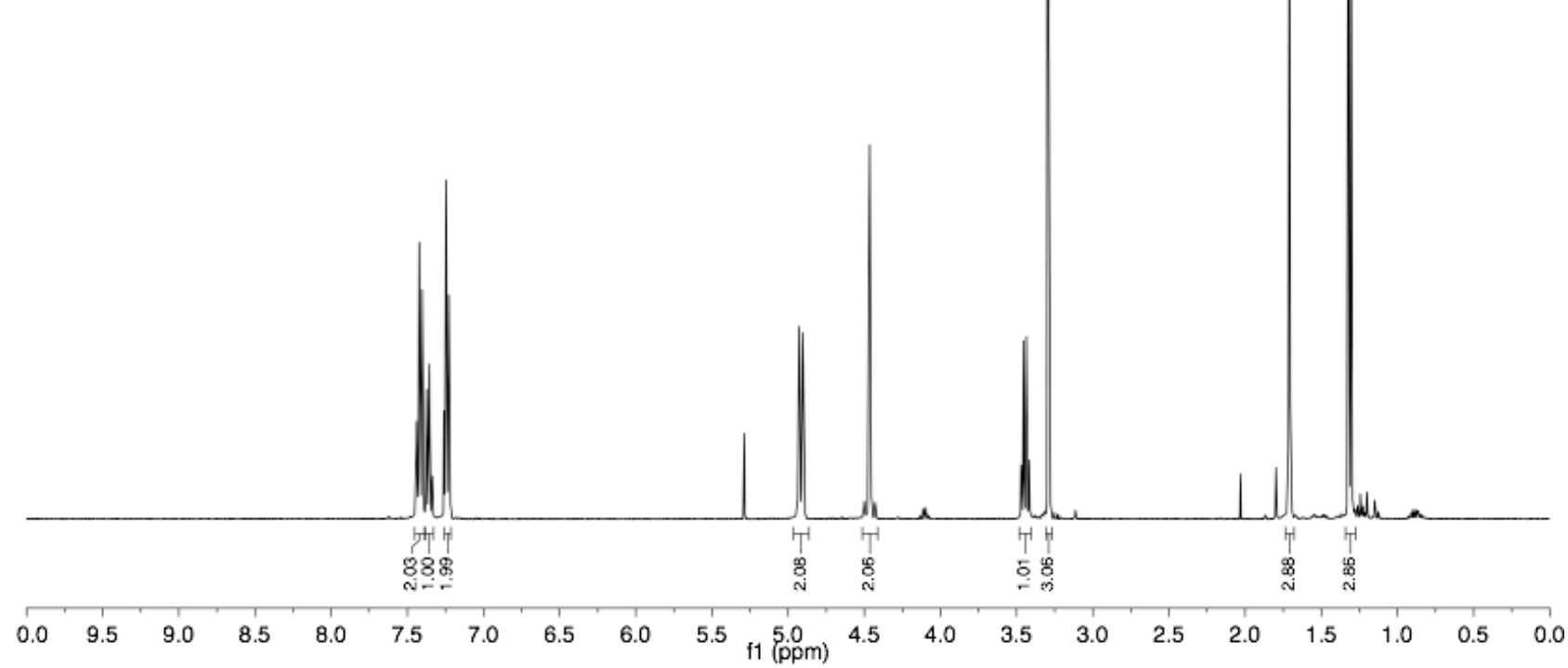


$\mathrm{CDCl}_{3}, 400 \mathrm{MHz}$

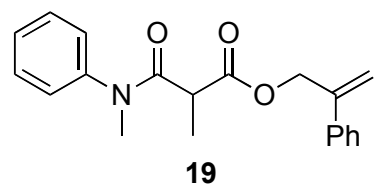

$\mathrm{CDCl}_{3}, 400 \mathrm{MHz}$
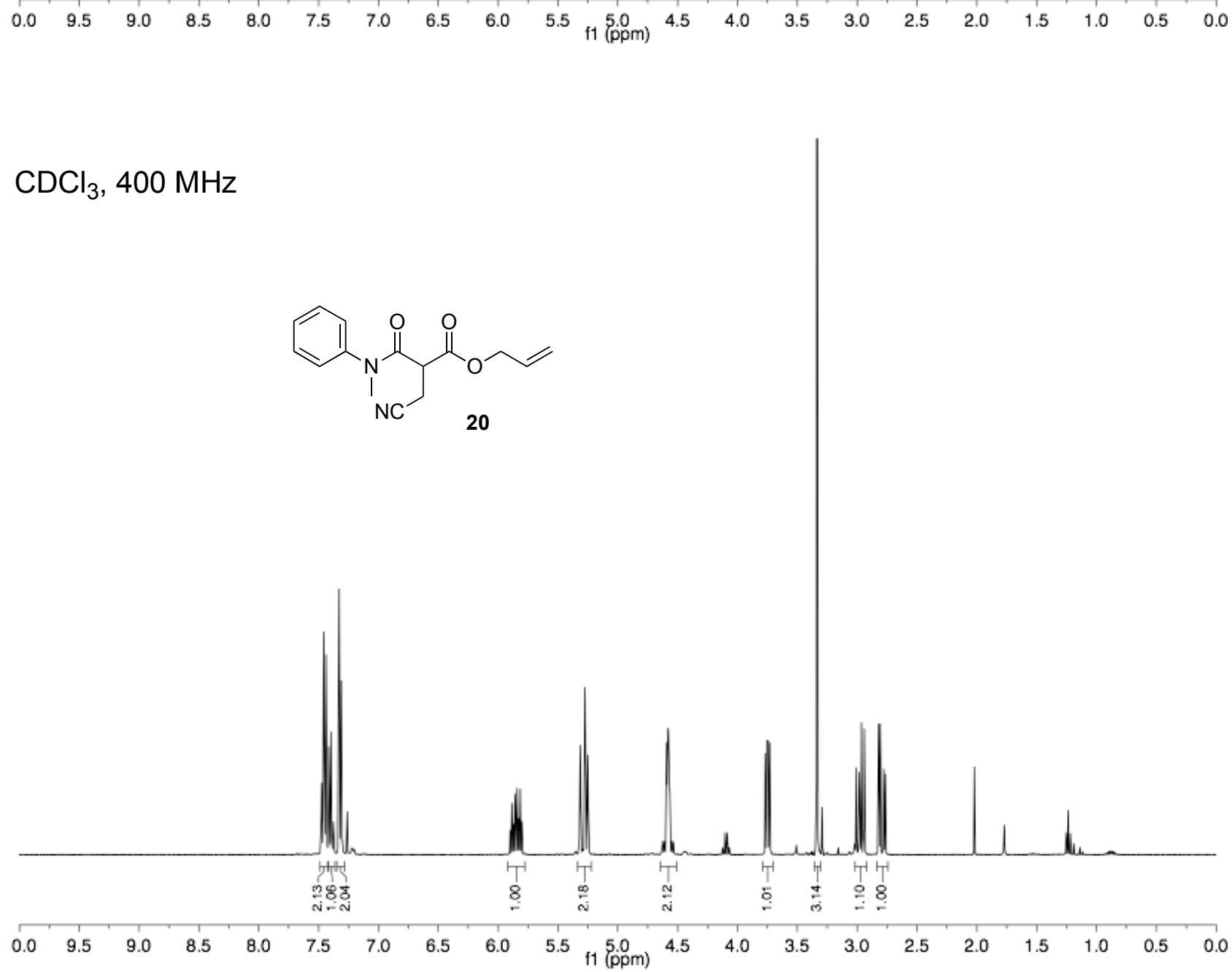
$\mathrm{CDCl}_{3}, 400 \mathrm{MHz}$<smiles>C=CCOC(=O)C(C(=O)N(C)c1ccccc1)C(C)C</smiles>

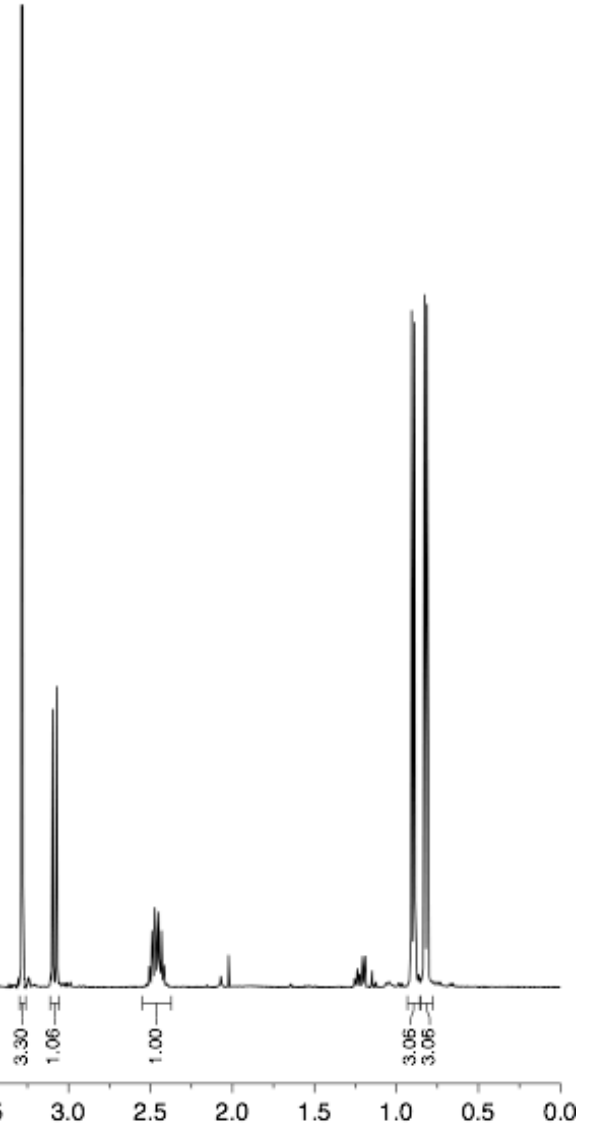

$\mathrm{CDCl}_{3}, 400 \mathrm{MHz}$

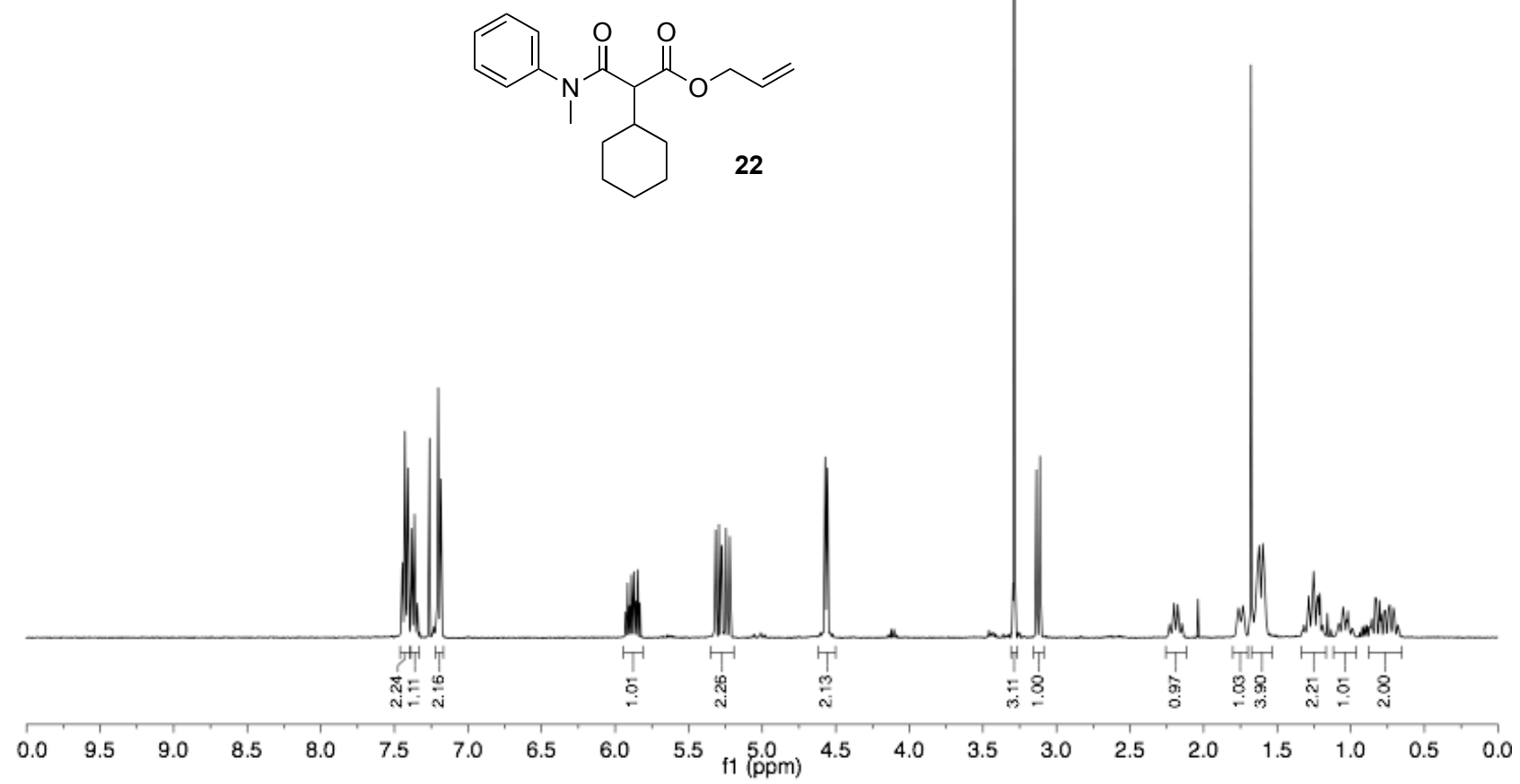


$\mathrm{CDCl}_{3}, 400 \mathrm{MHz}$
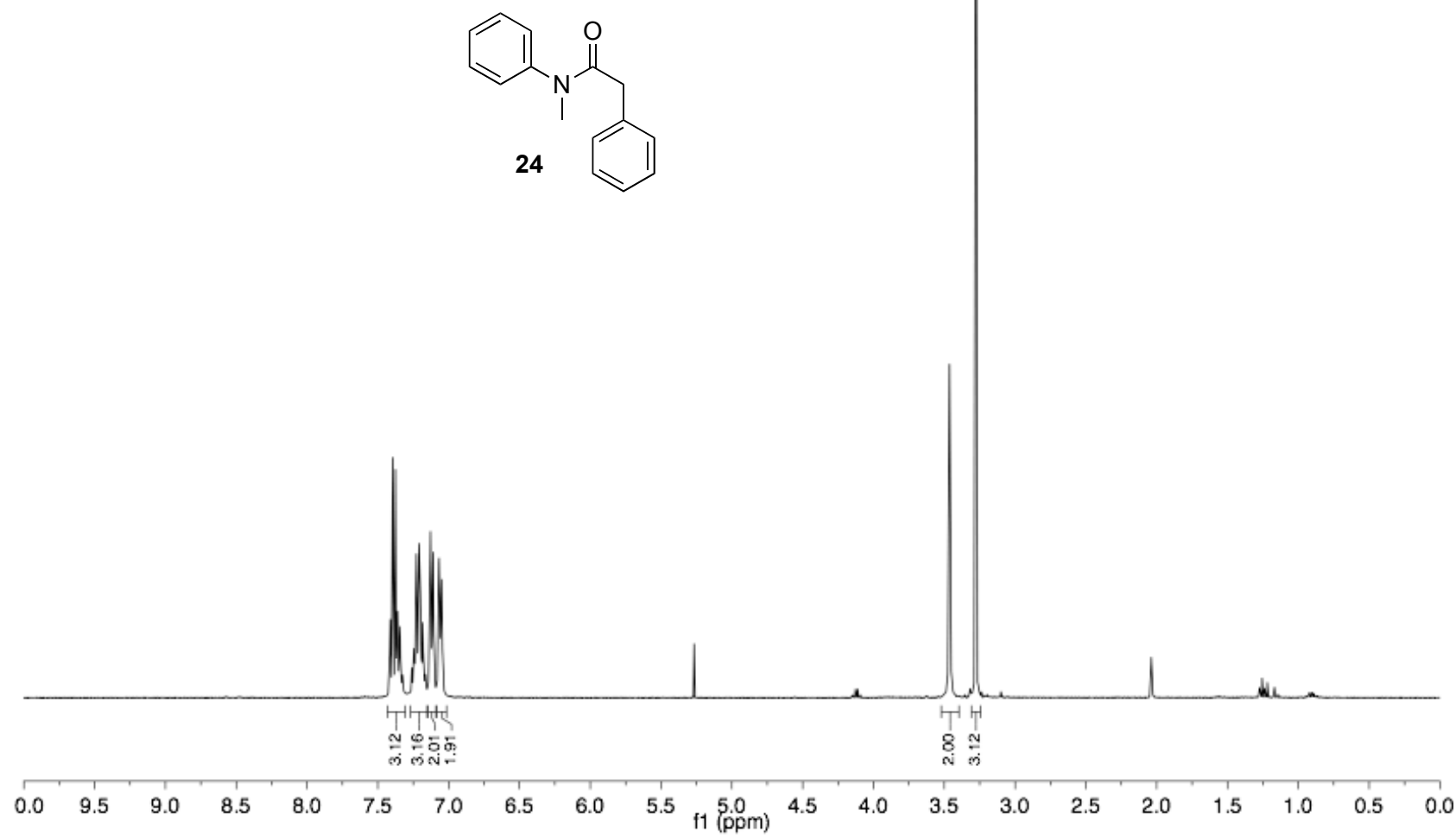

$\mathrm{CDCl}_{3}, 400 \mathrm{MHz}$
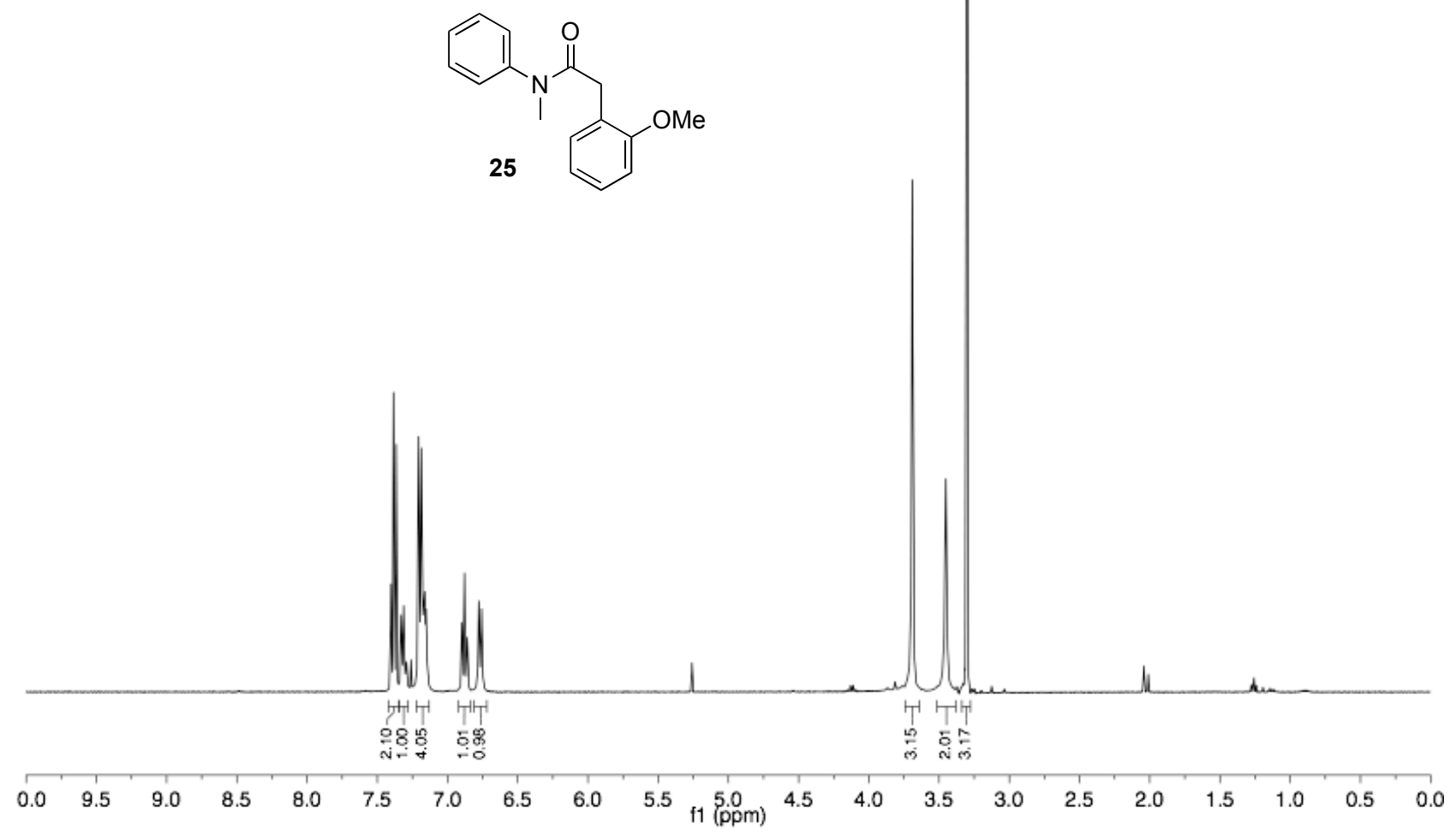

S-54 
$\mathrm{CDCl}_{3}, 400 \mathrm{MHz}$
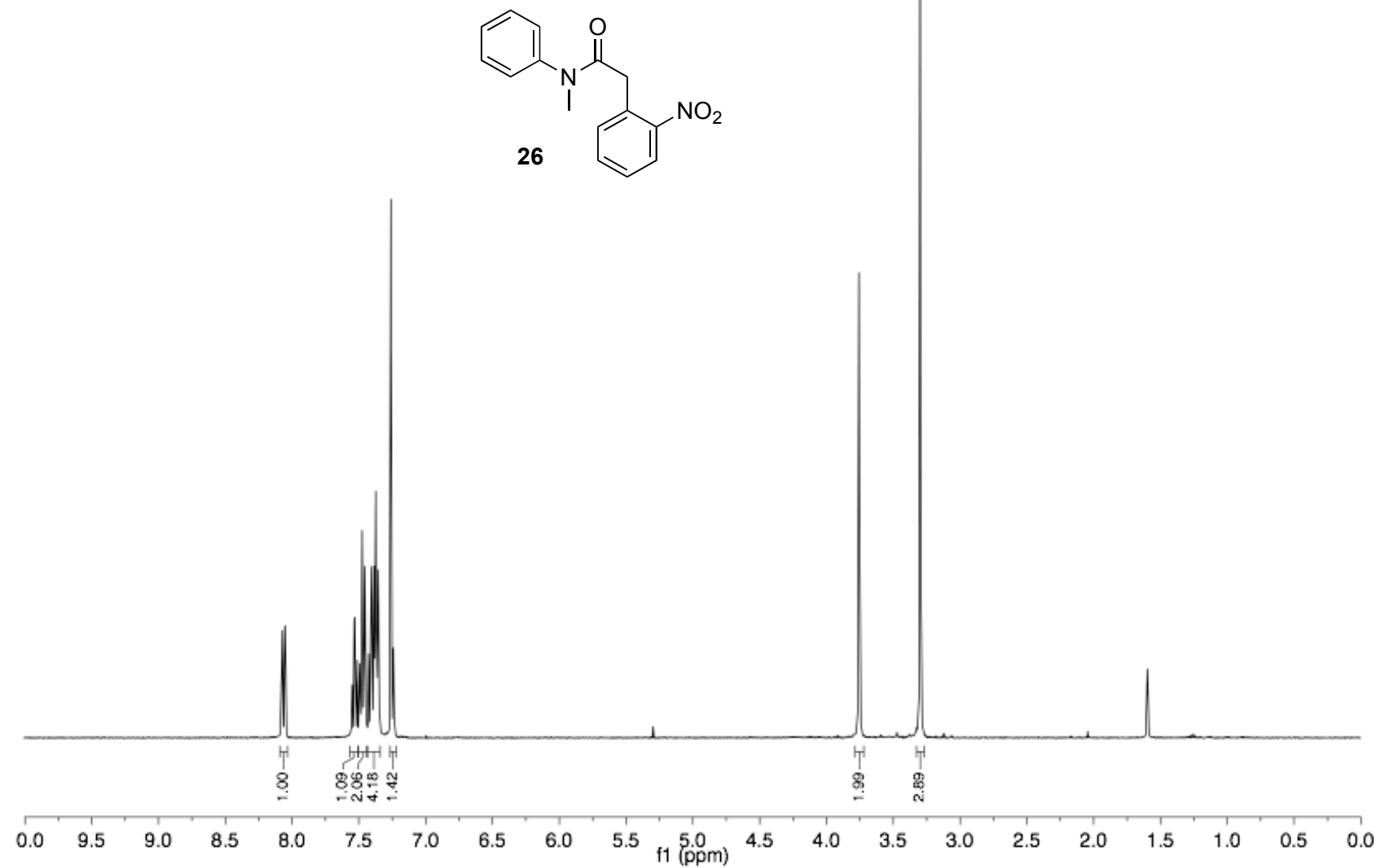

$\mathrm{CDCl}_{3}, 400 \mathrm{MHz}$
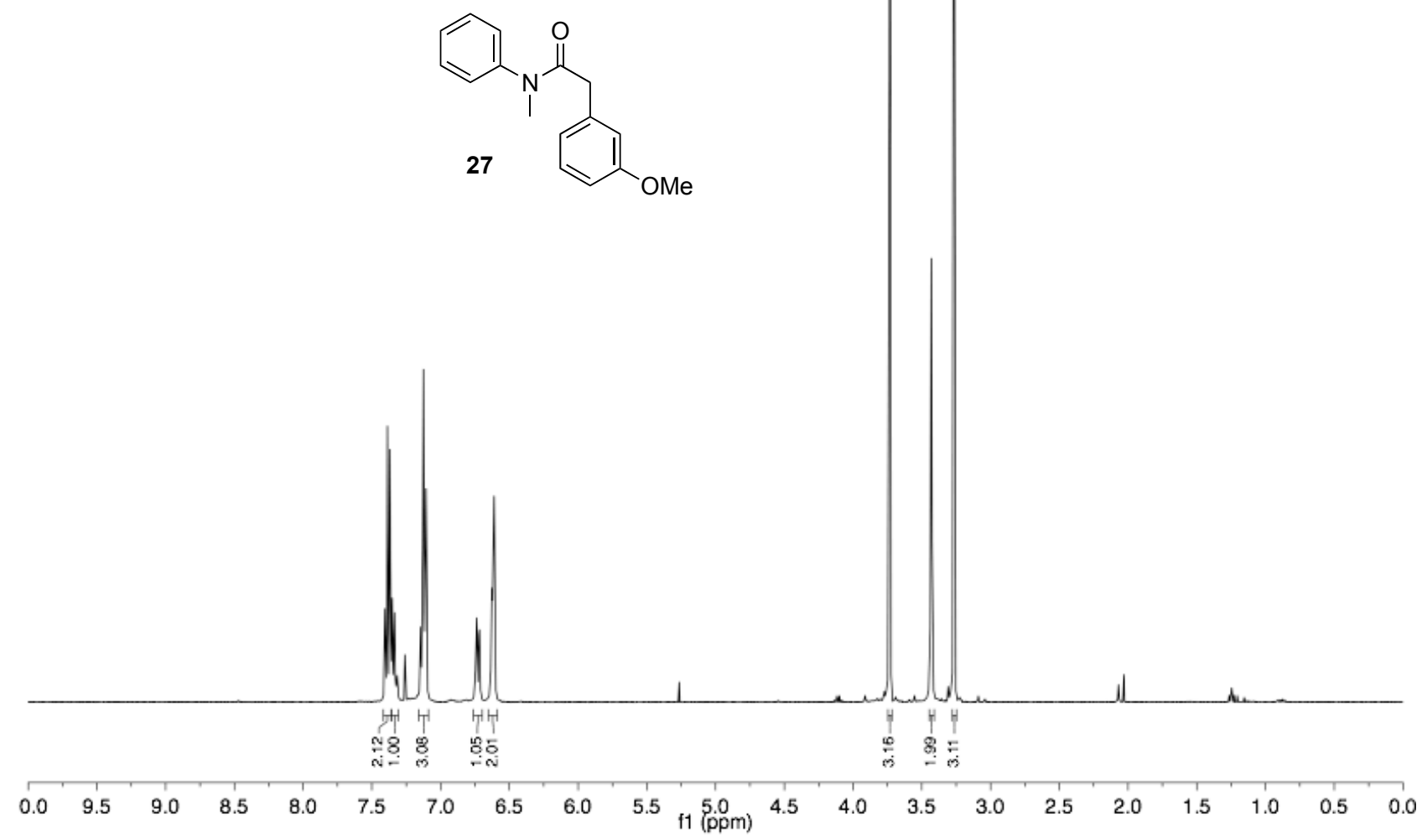

S-55 
$\mathrm{CDCl}_{3}, 400 \mathrm{MHz}$
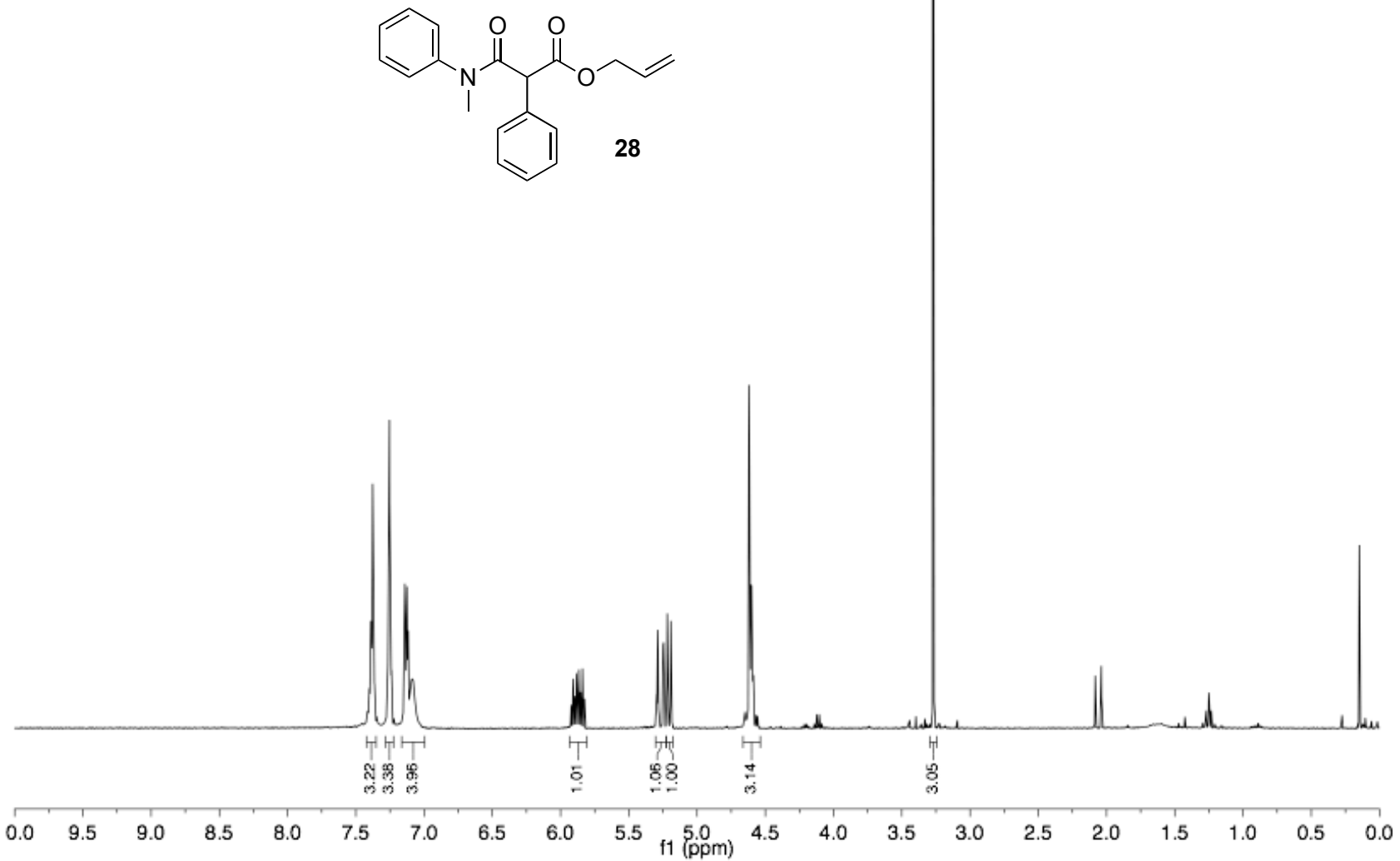

$\mathrm{CDCl}_{3}, 400 \mathrm{MHz}$
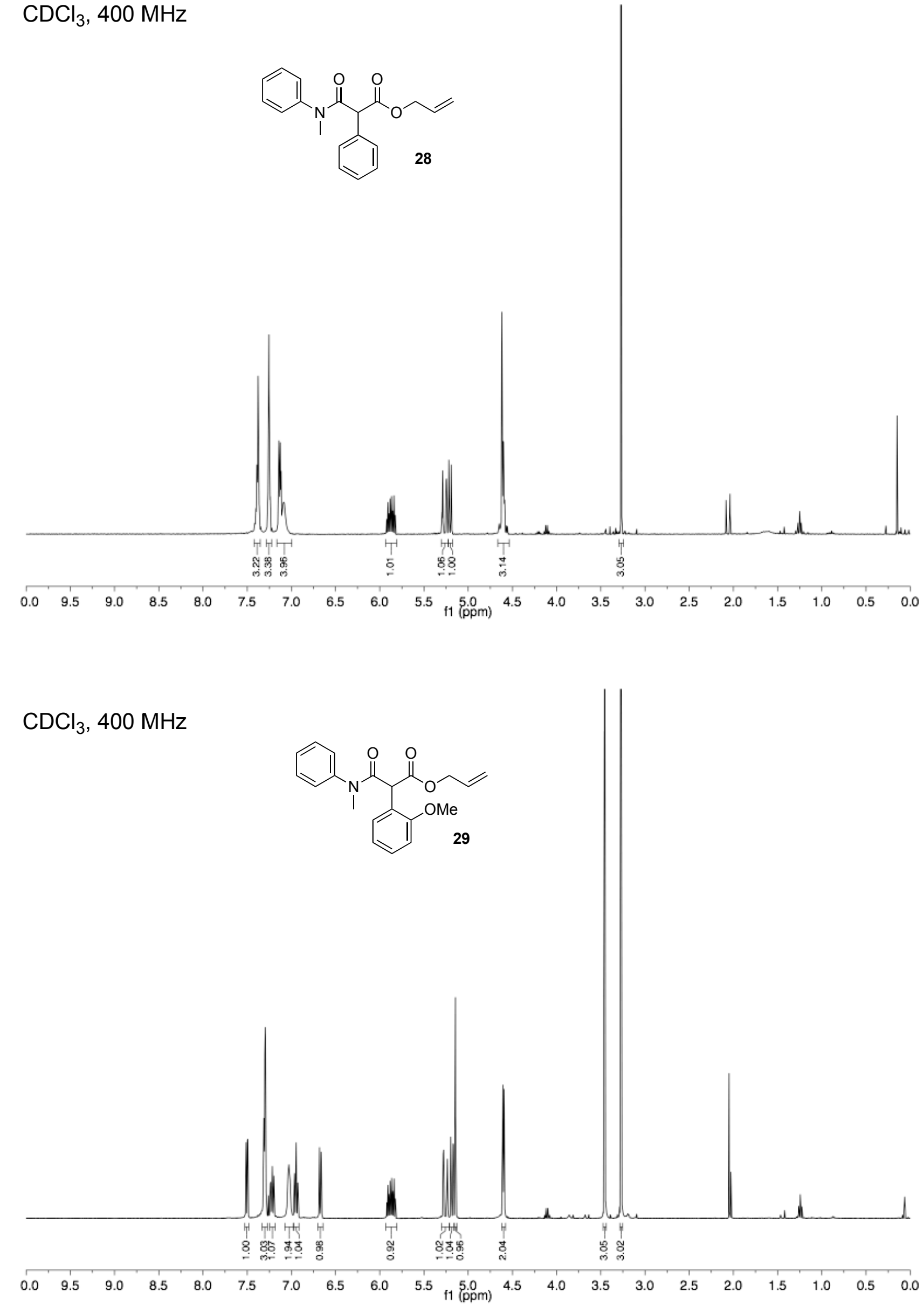
$\mathrm{CDCl}_{3}, 400 \mathrm{MHz}$
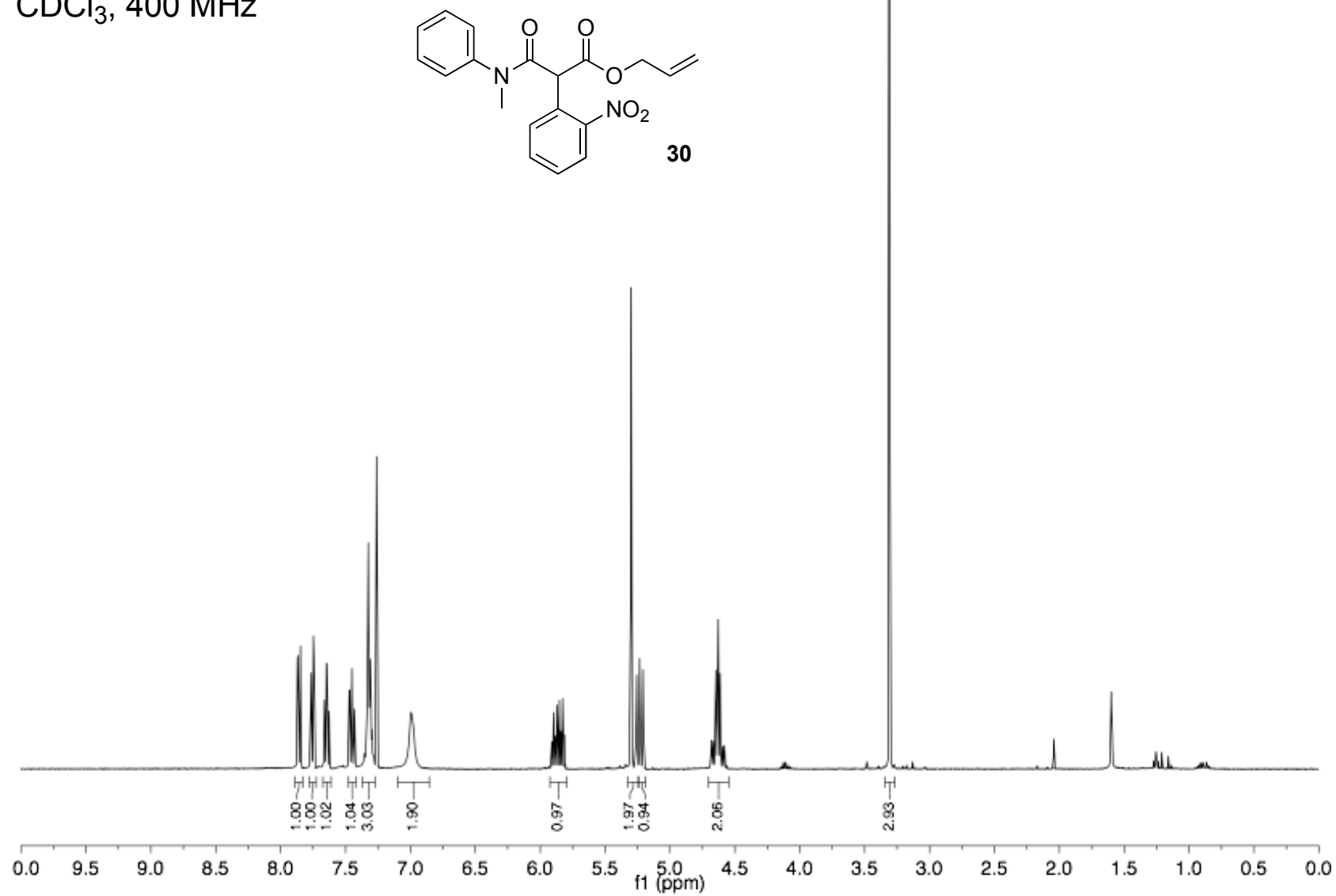

$\mathrm{CDCl}_{3}, 400 \mathrm{MHz}$

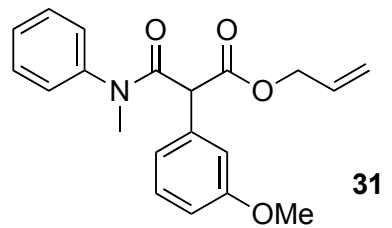

31

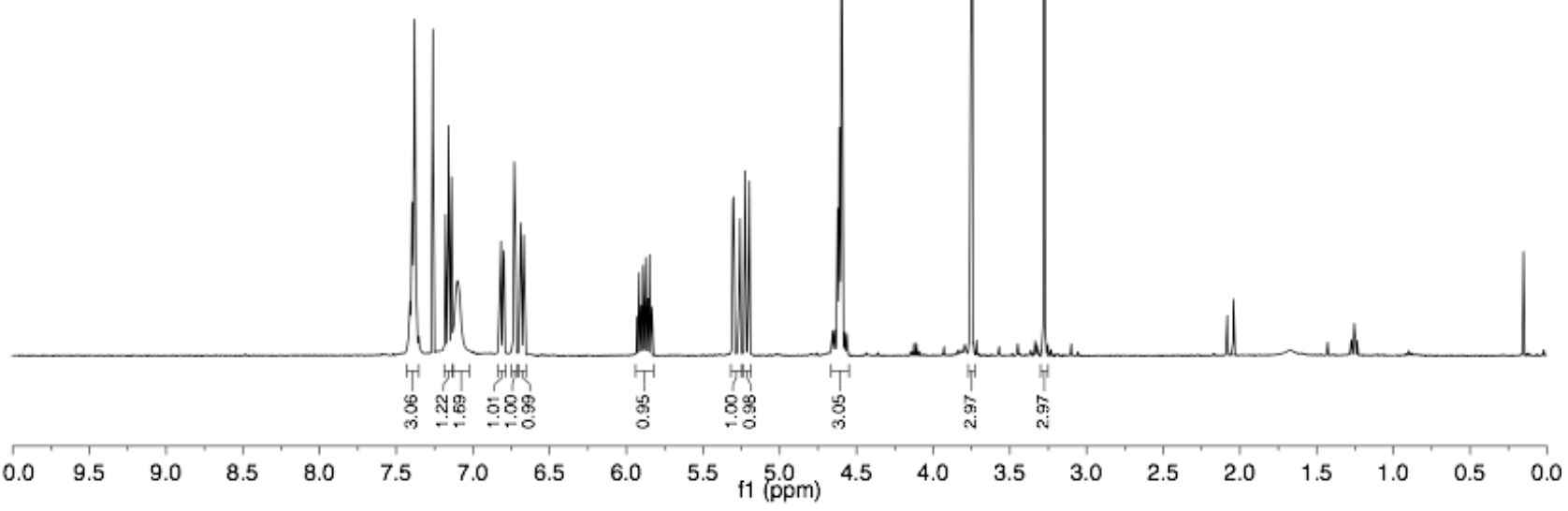


$\mathrm{CDCl}_{3}, 400 \mathrm{MHz}$
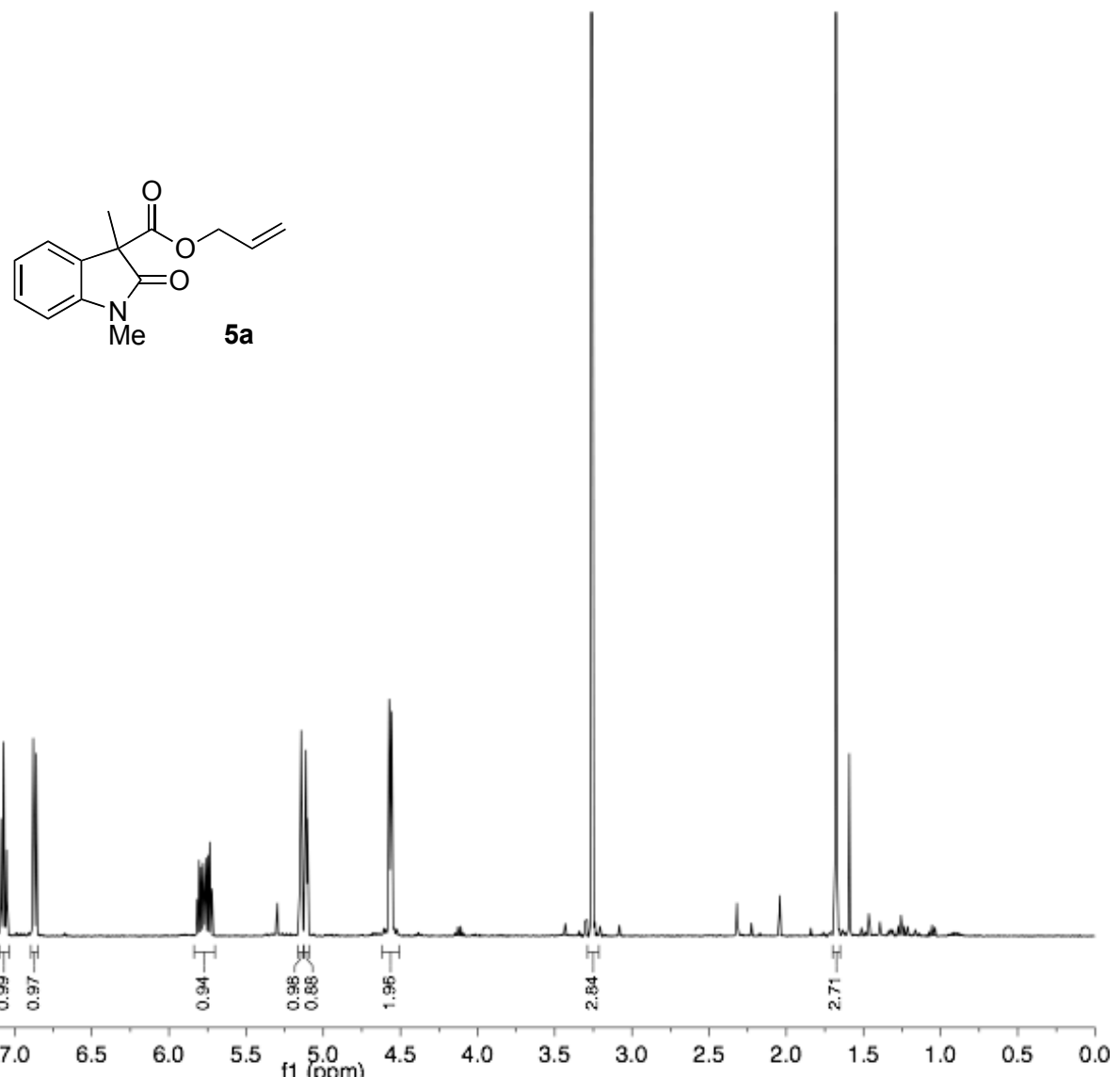

$\mathrm{CDCl}_{3}, 400 \mathrm{MHz}$
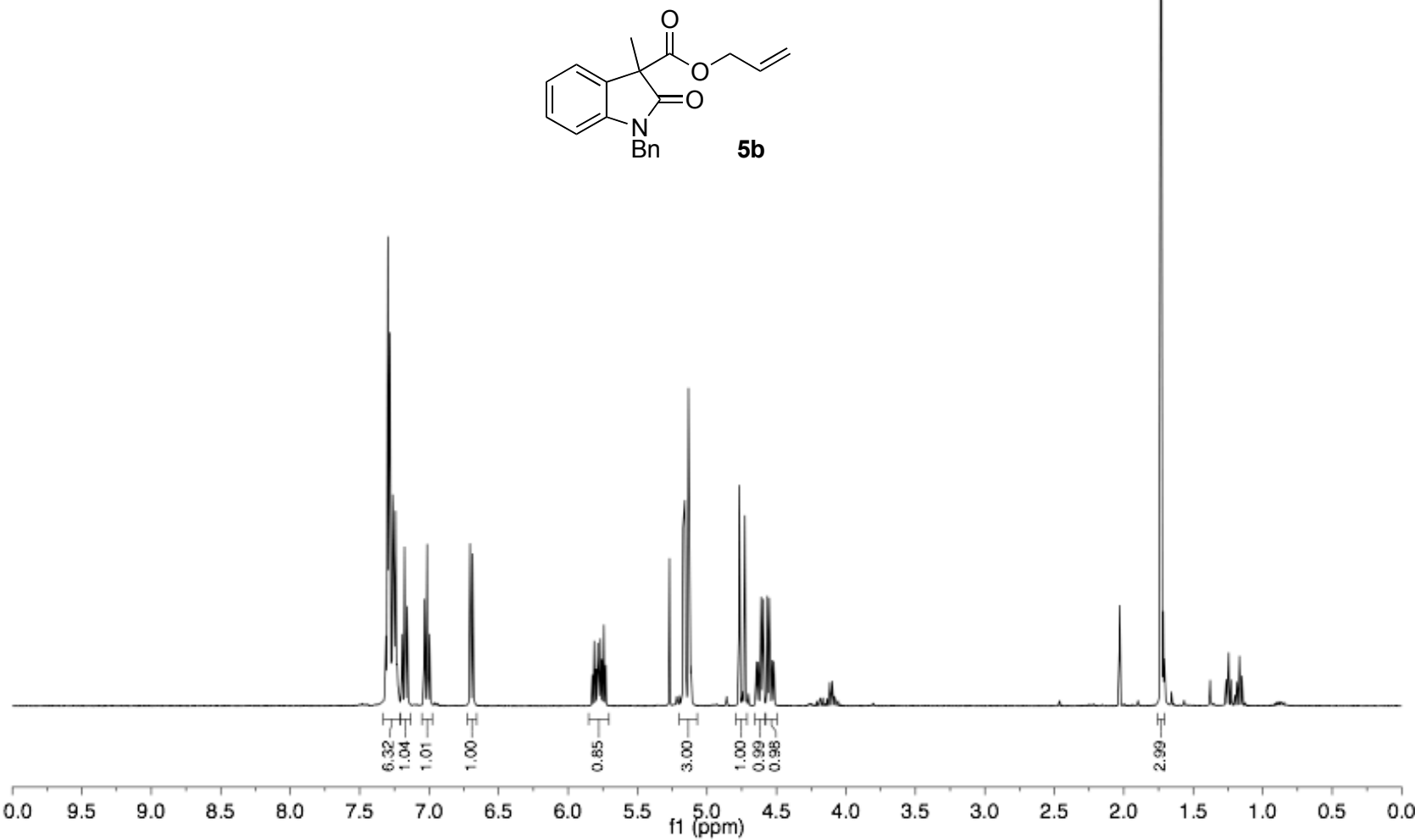
$\mathrm{CDCl}_{3}, 400 \mathrm{MHz}$

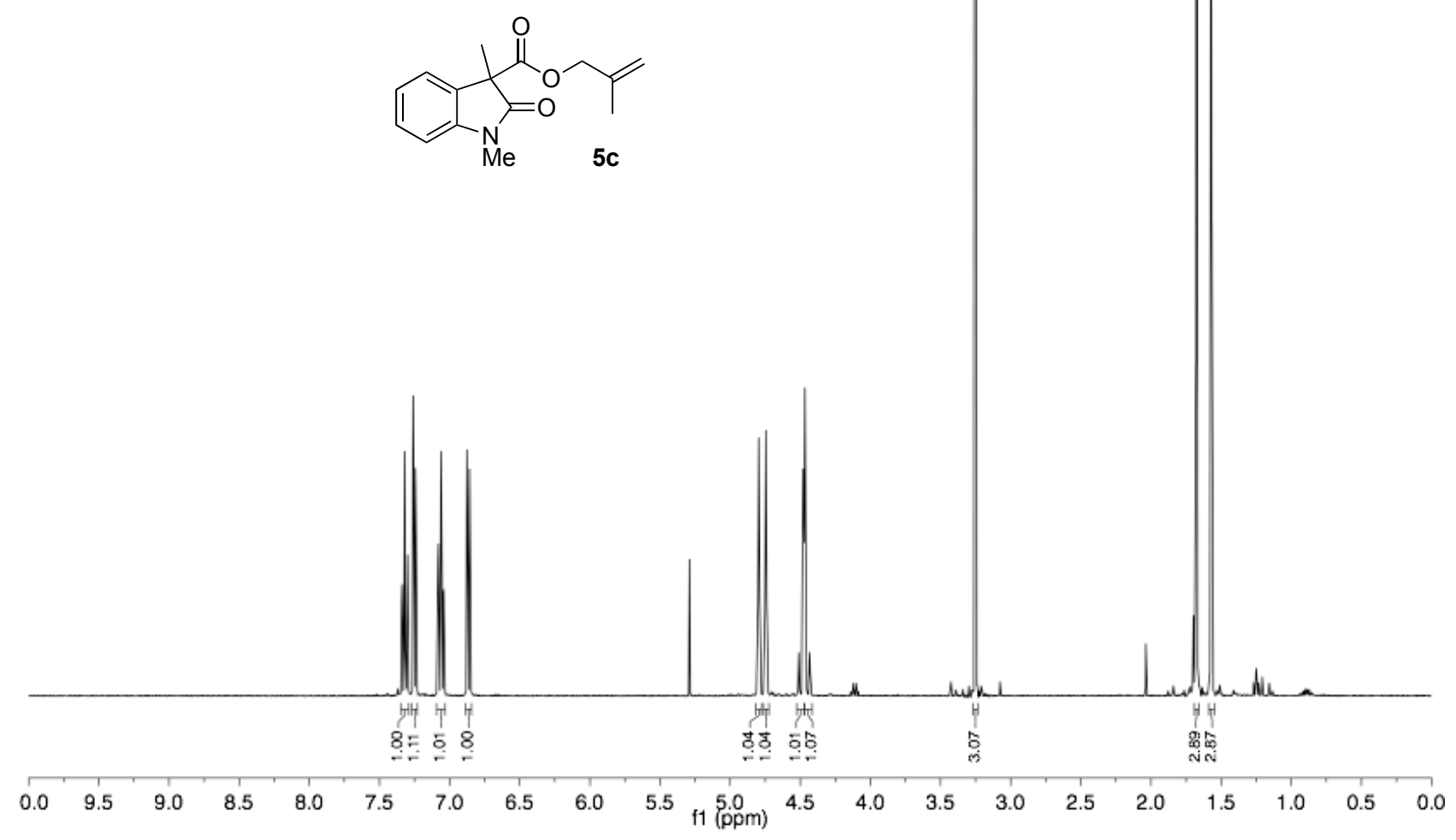

$\mathrm{CDCl}_{3}, 400 \mathrm{MHz}$
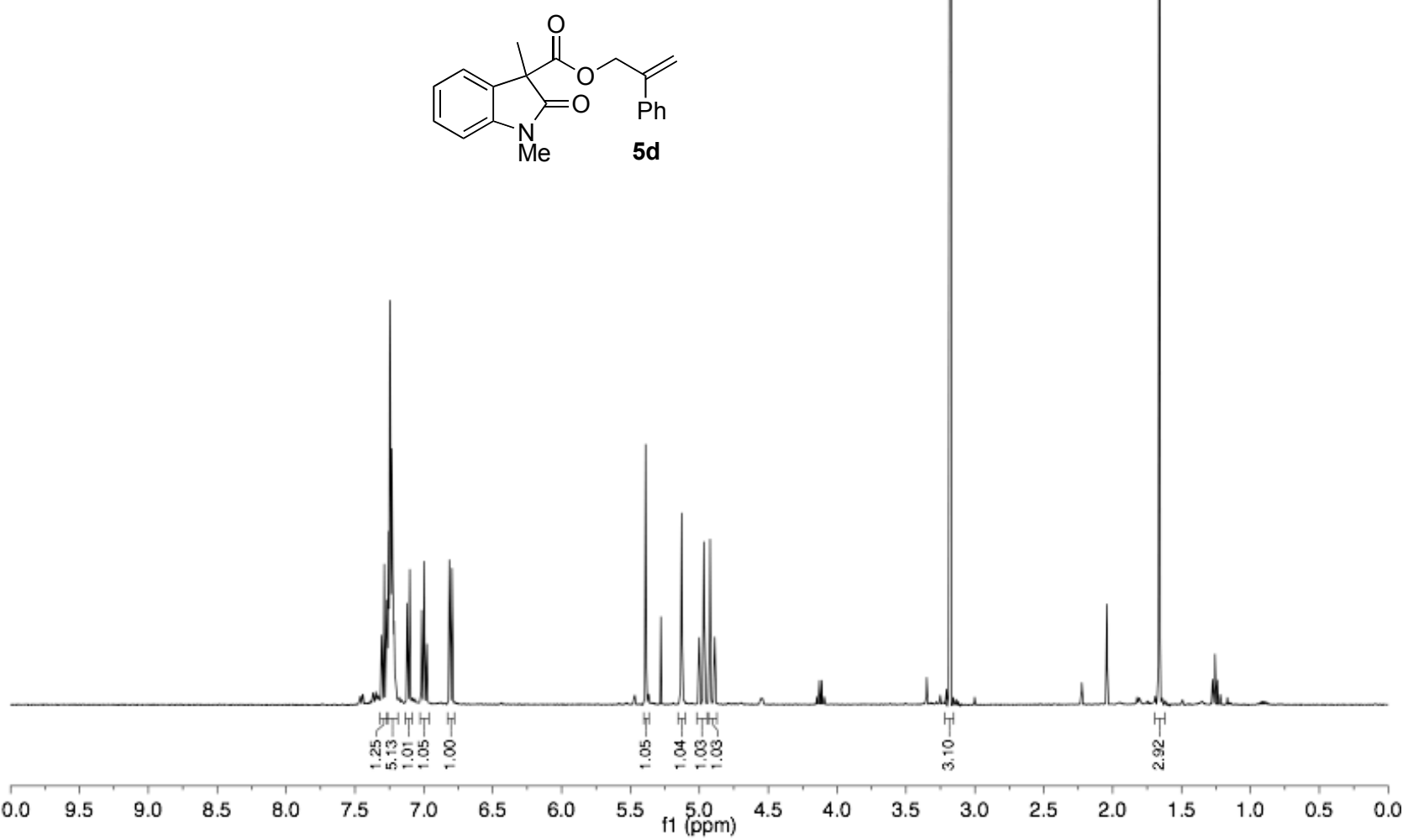
$\mathrm{CDCl}_{3}, 400 \mathrm{MHz}$

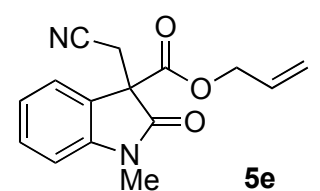

$\mathrm{CDCl}_{3}, 400 \mathrm{MHz}$
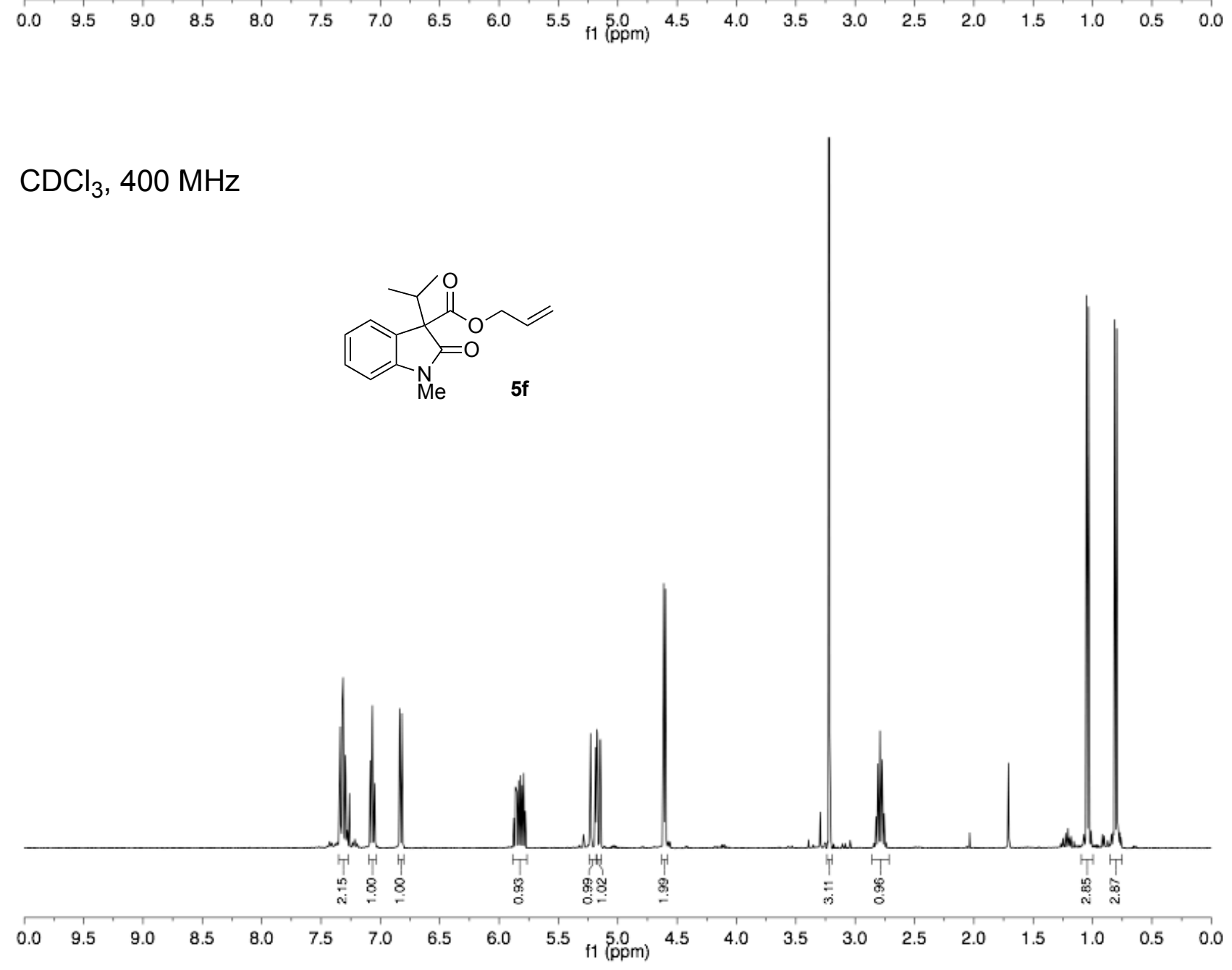
$\mathrm{CDCl}_{3}, 400 \mathrm{MHz}$

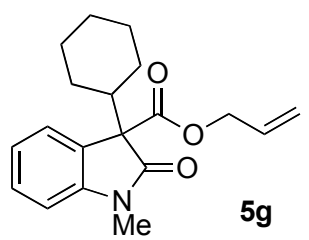

$\mathrm{CDCl}_{3}, 400 \mathrm{MHz}$
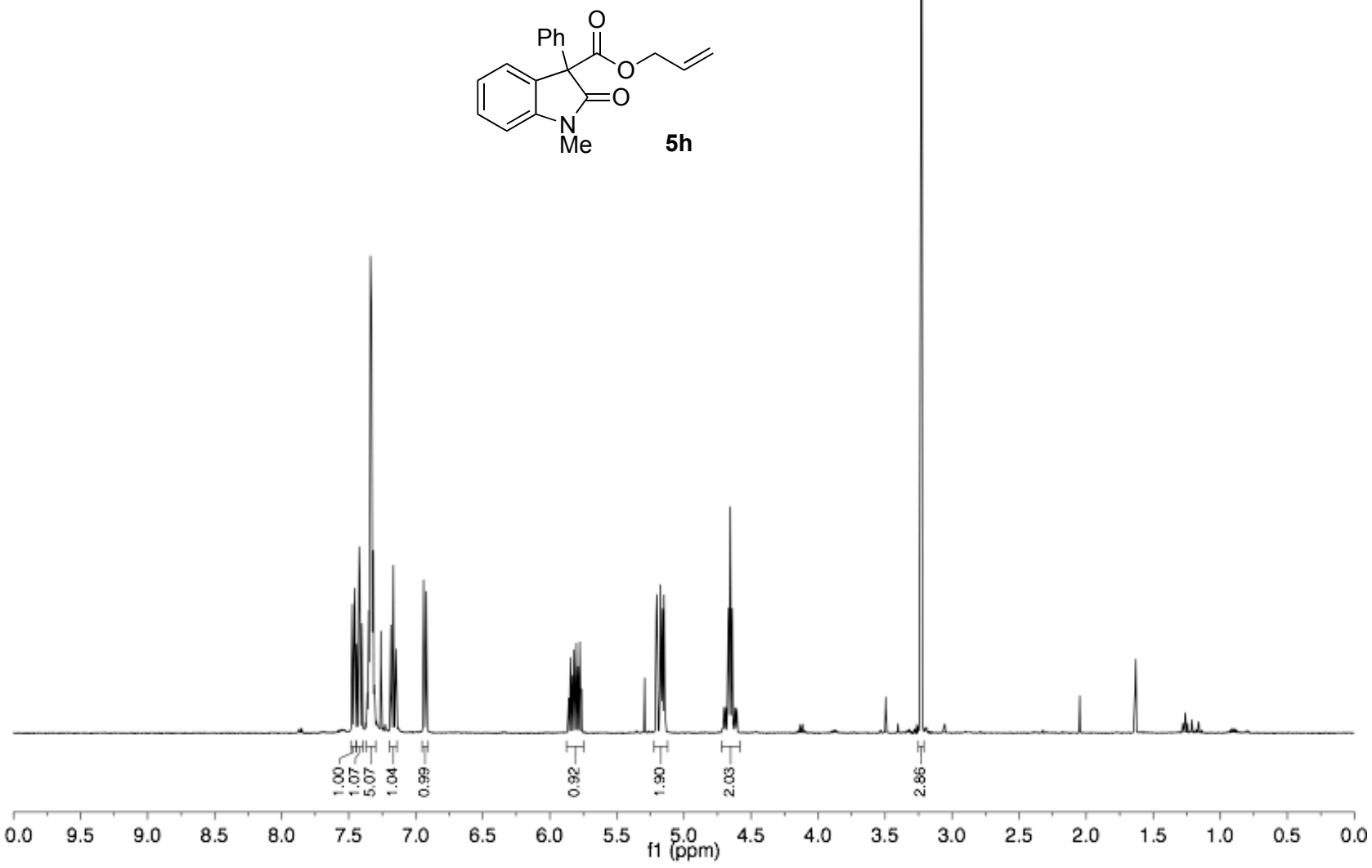
$\mathrm{CDCl}_{3}, 400 \mathrm{MHz}$
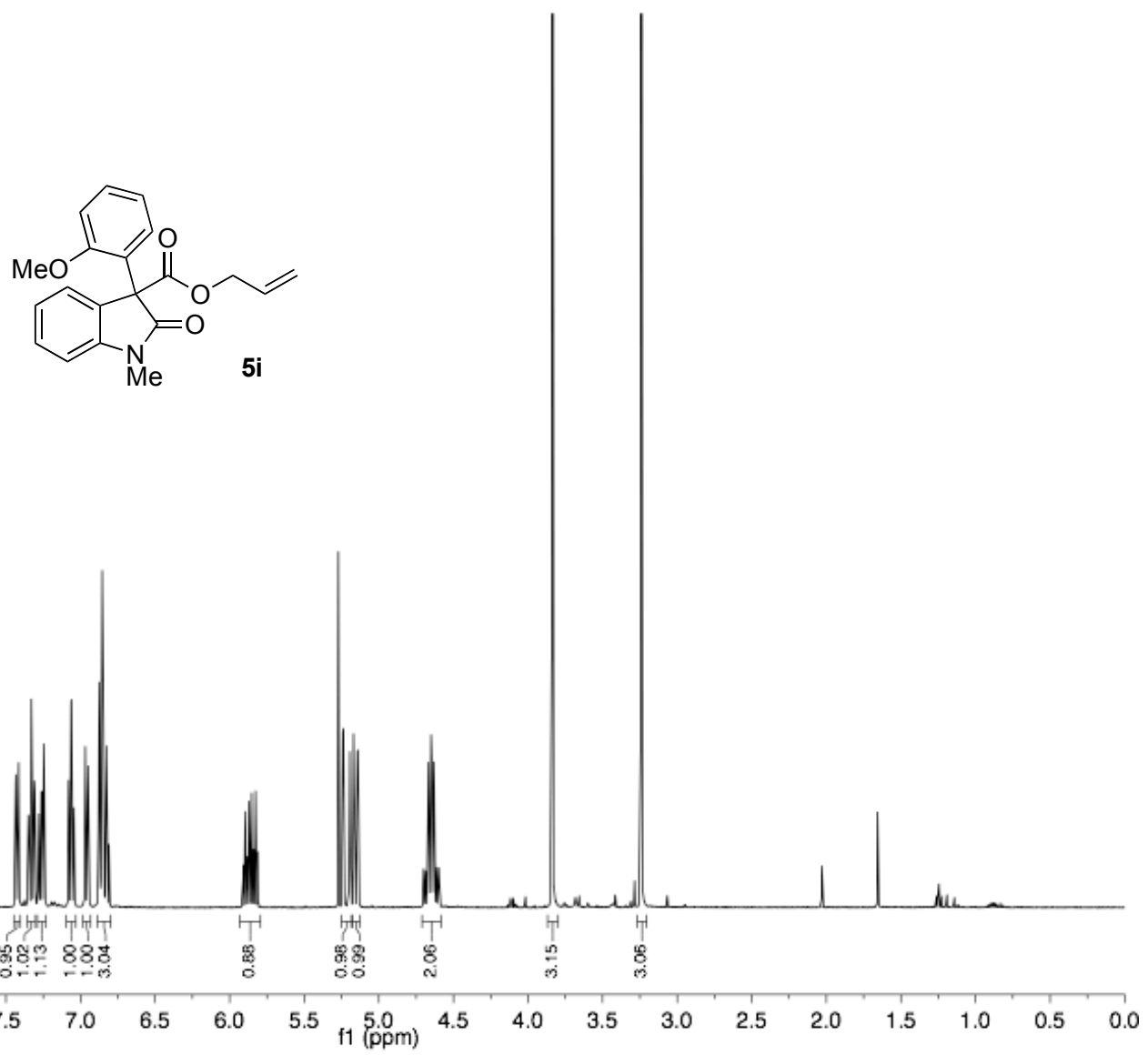

$\mathrm{CDCl}_{3}, 400 \mathrm{MHz}$
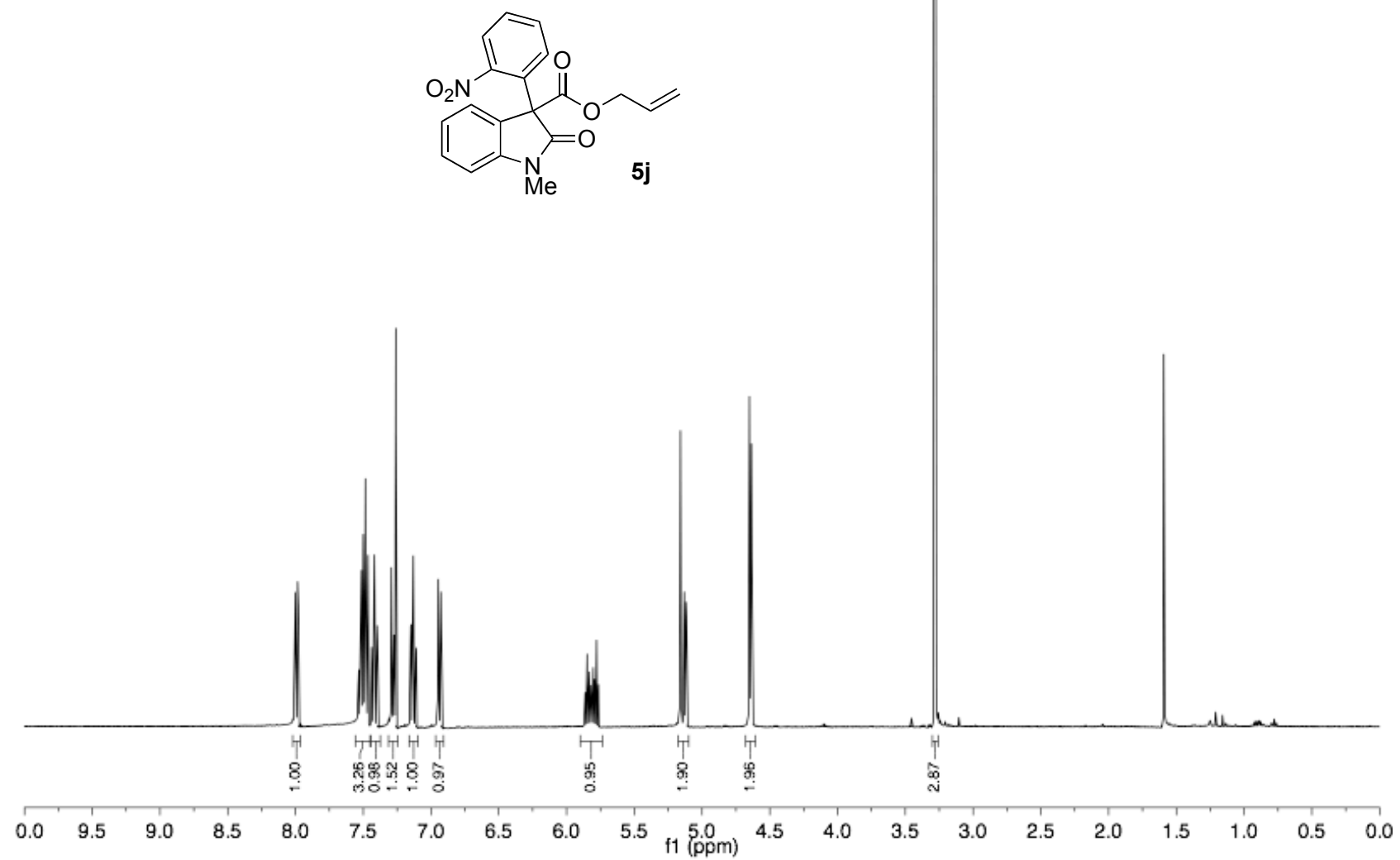
$\mathrm{CDCl}_{3}, 400 \mathrm{MHz}$

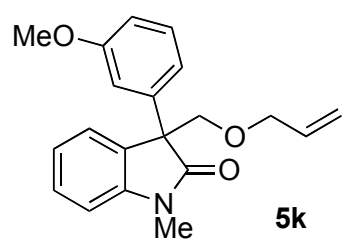

$\mathrm{CDCl}_{3}, 400 \mathrm{MHz}$

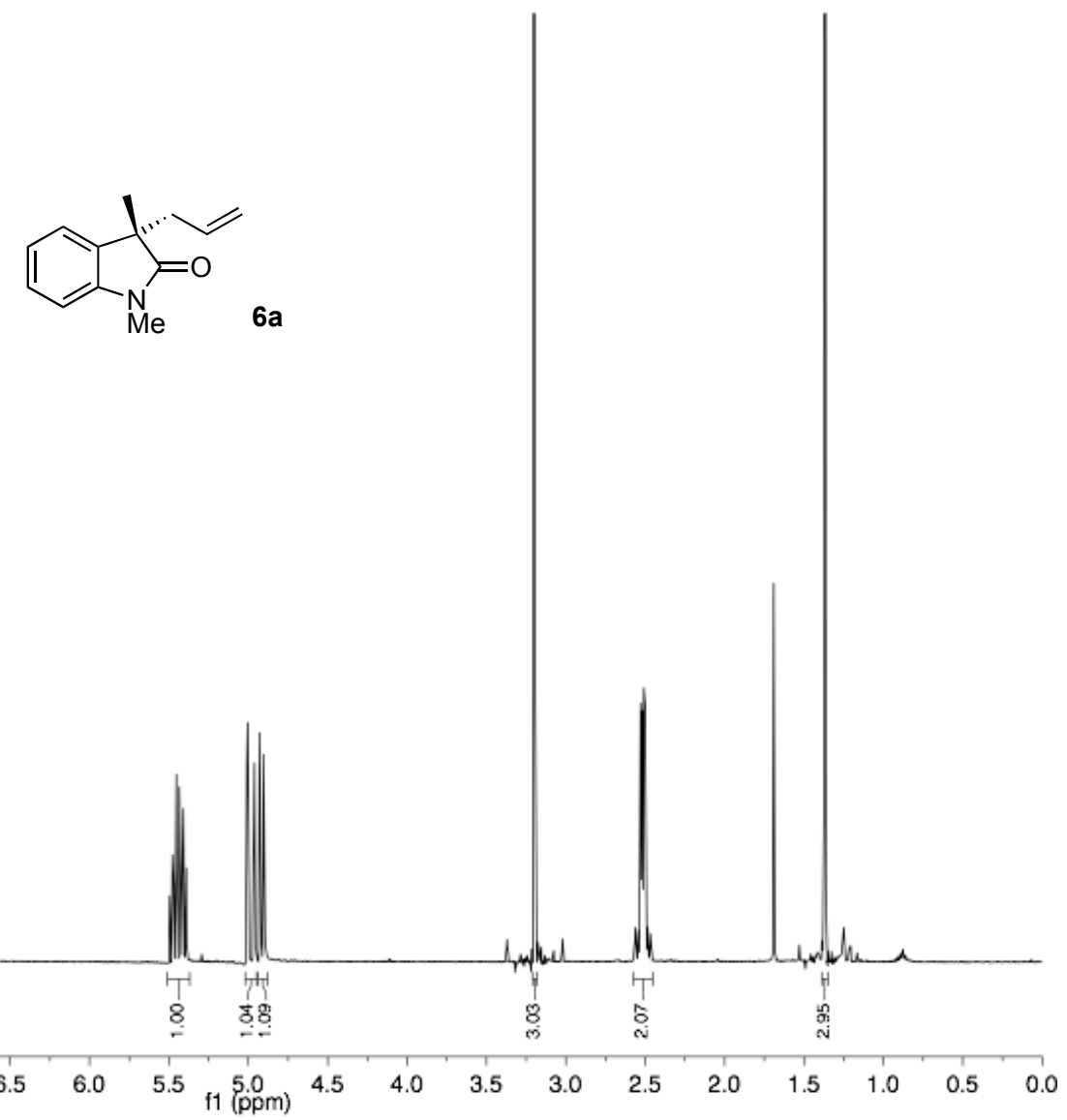




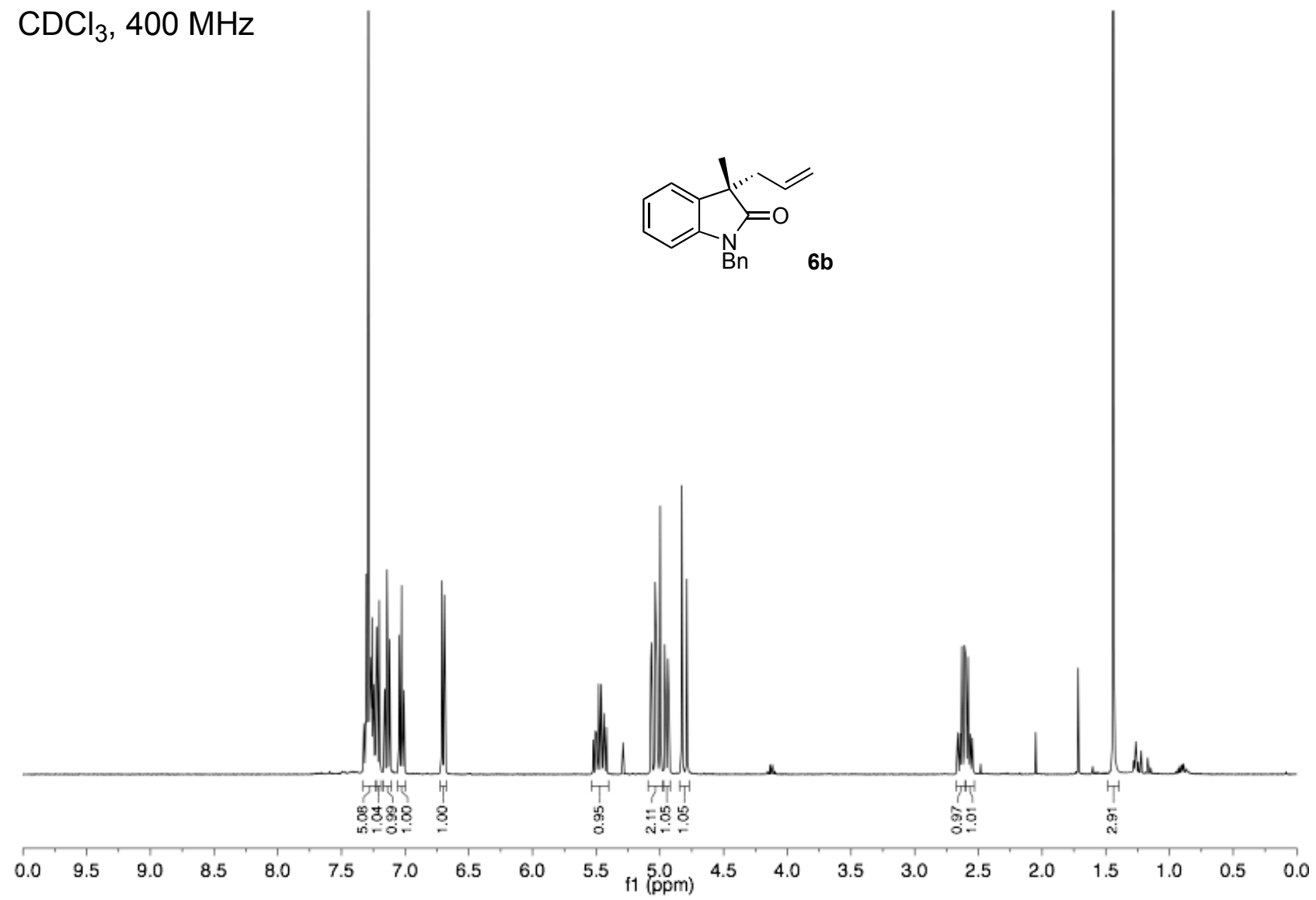

$\mathrm{CDCl}_{3}, 400 \mathrm{MHz}$

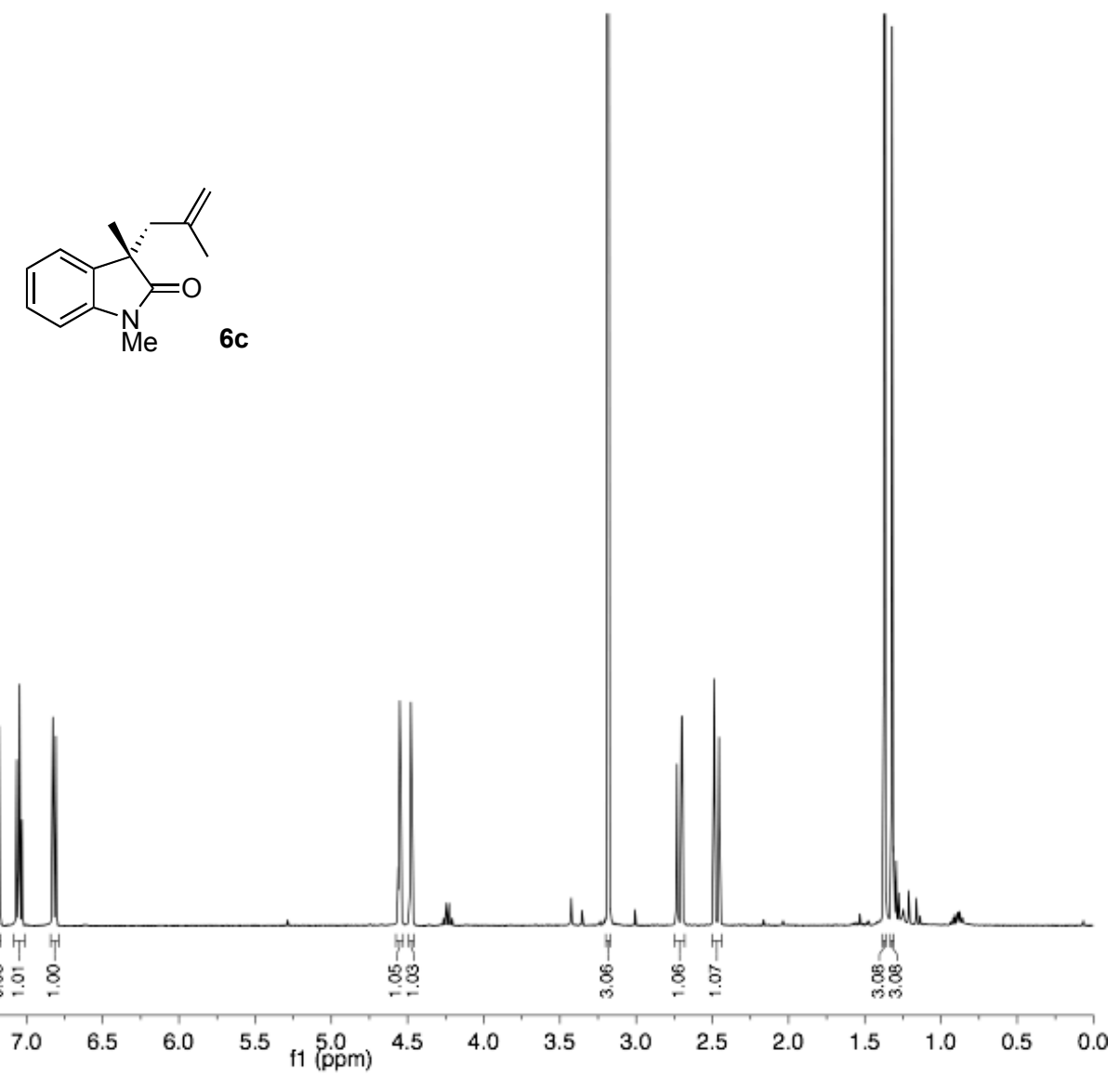


$\mathrm{CDCl}_{3}, 400 \mathrm{MHz}$

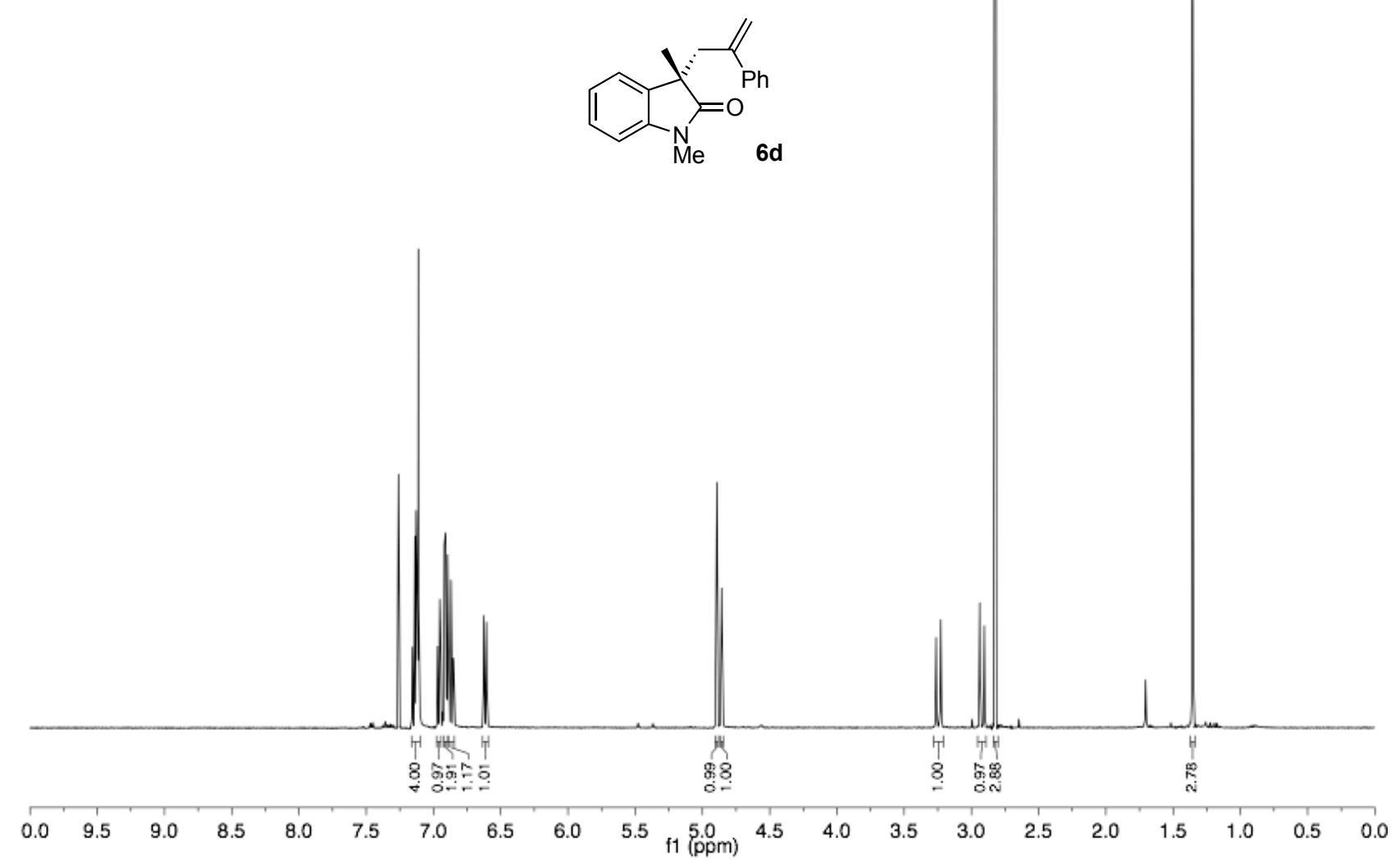

$\mathrm{CDCl}_{3}, 400 \mathrm{MHz}$

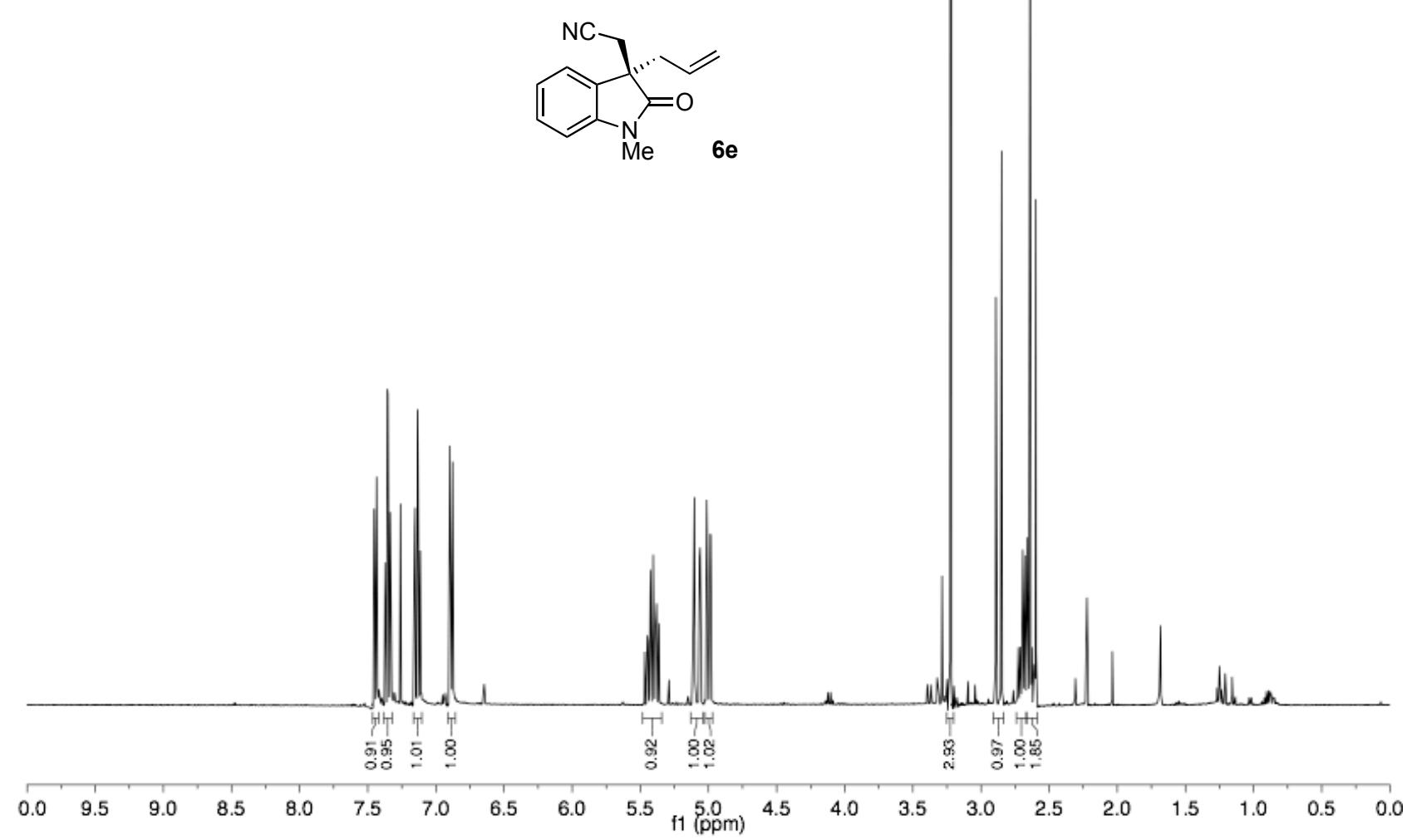


$\mathrm{CDCl}_{3}, 400 \mathrm{MHz}$
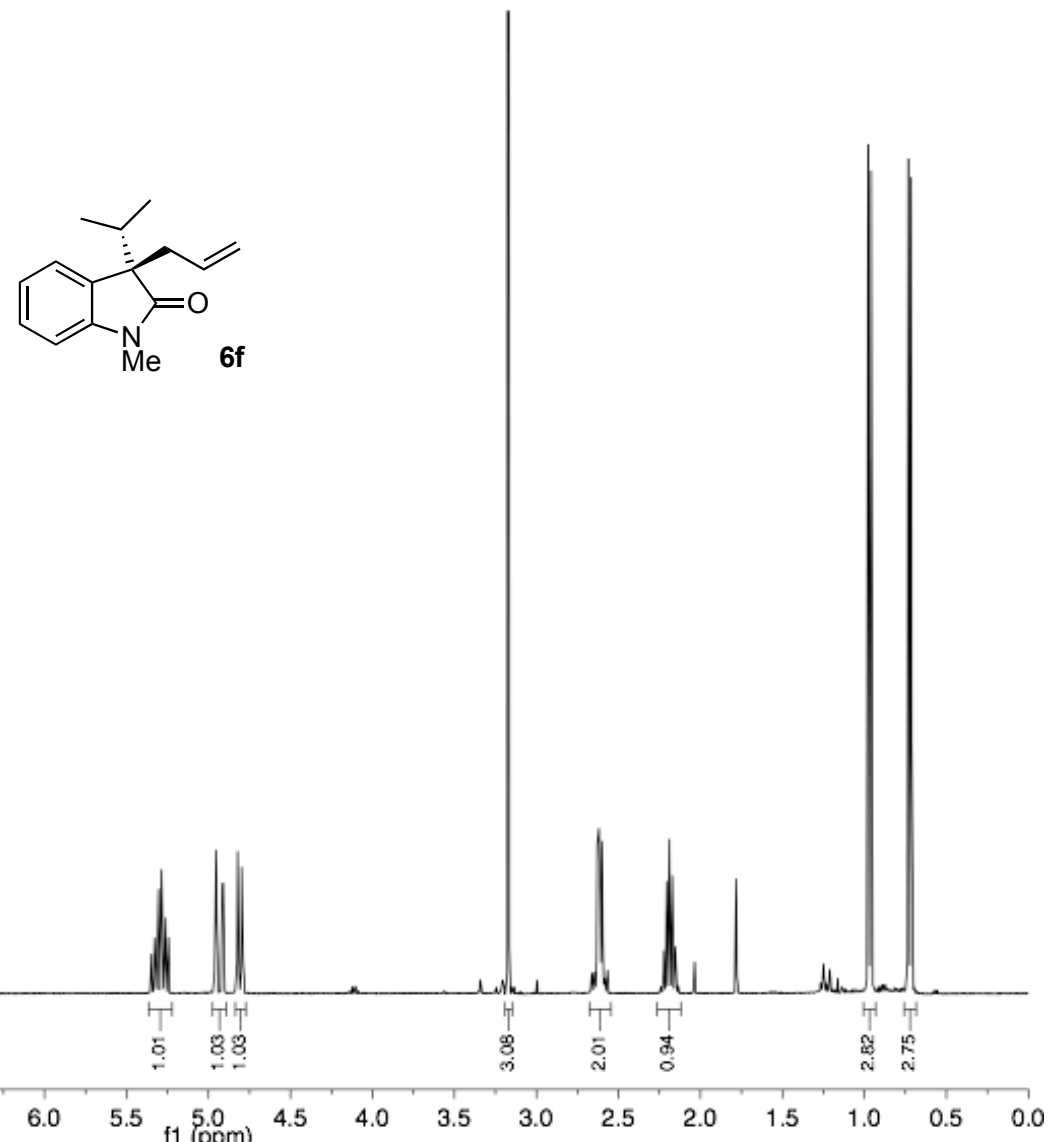

$\mathrm{CDCl}_{3}, 400 \mathrm{MHz}$
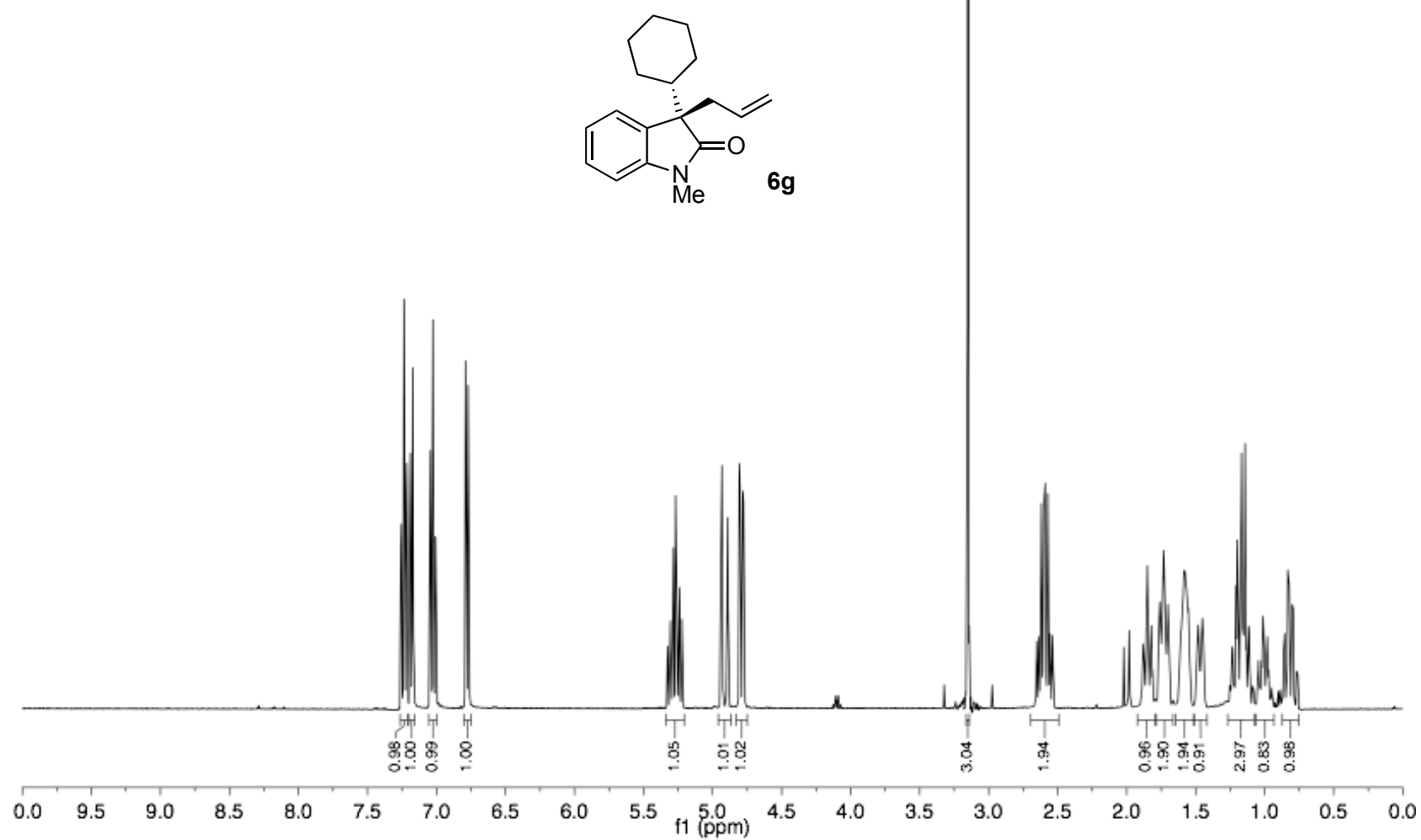
$\mathrm{CDCl}_{3}, 400 \mathrm{MHz}$
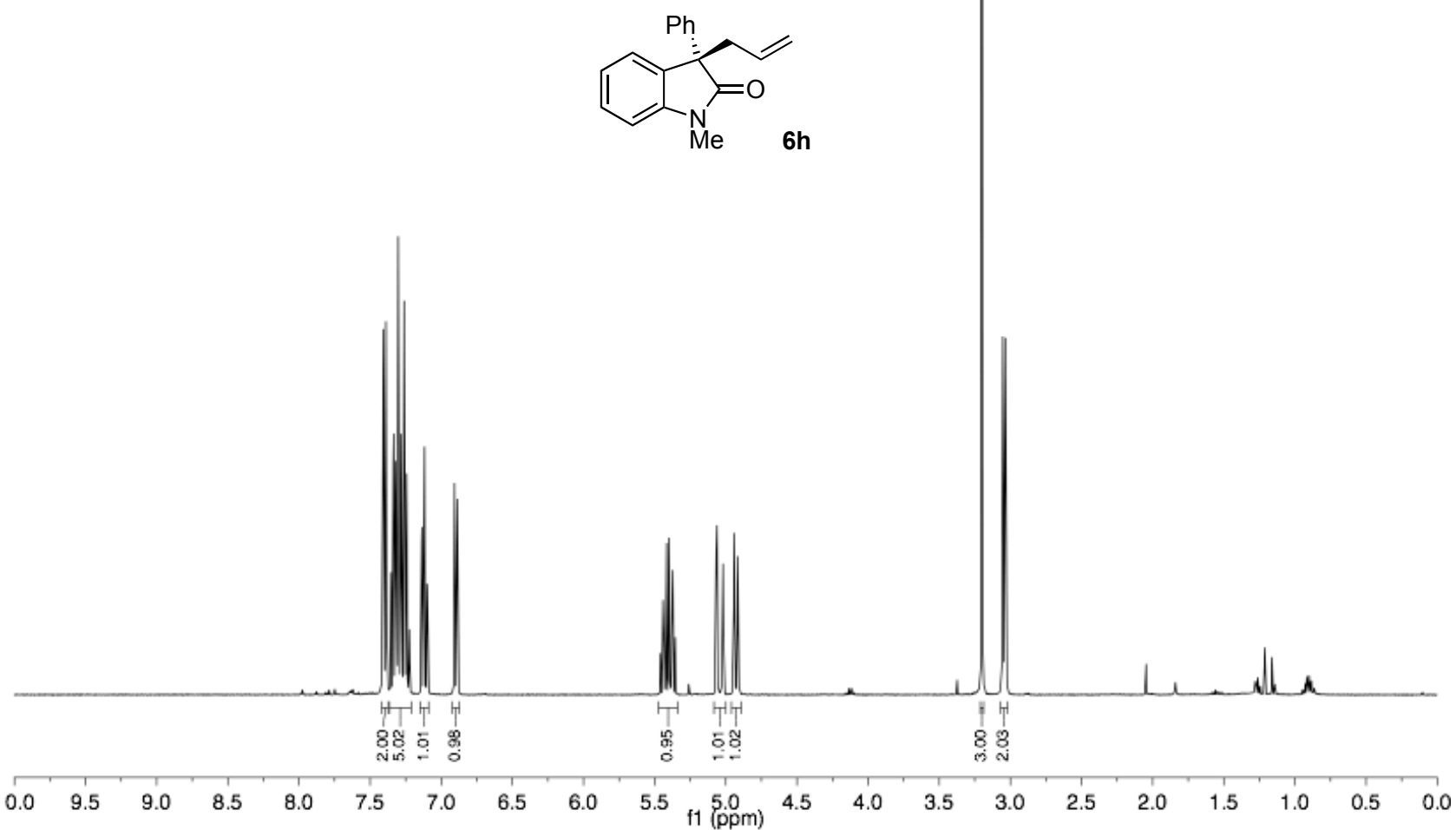

$\mathrm{CDCl}_{3}, 400 \mathrm{MHz}$
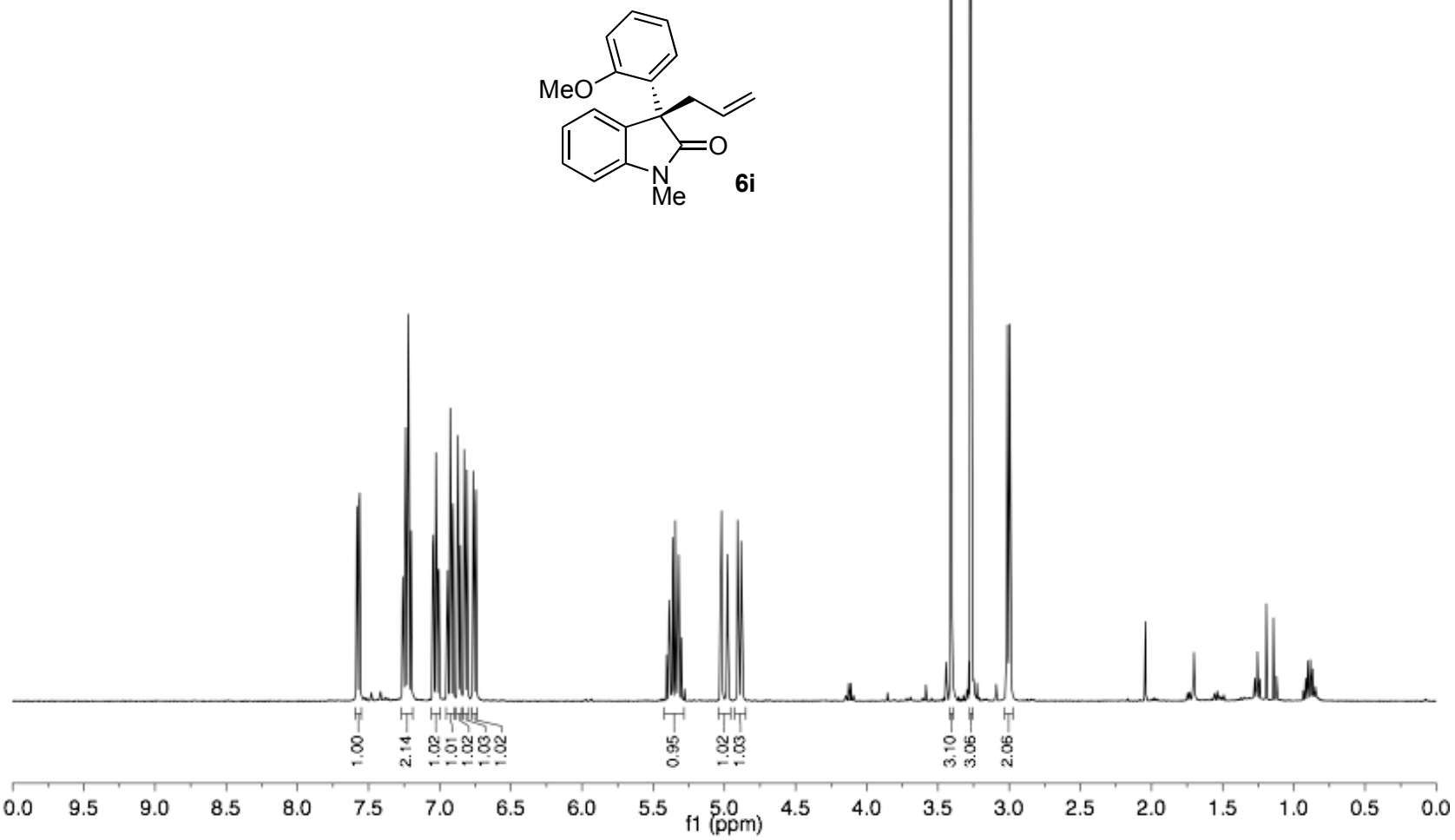
$\mathrm{CDCl}_{3}, 400 \mathrm{MHz}$

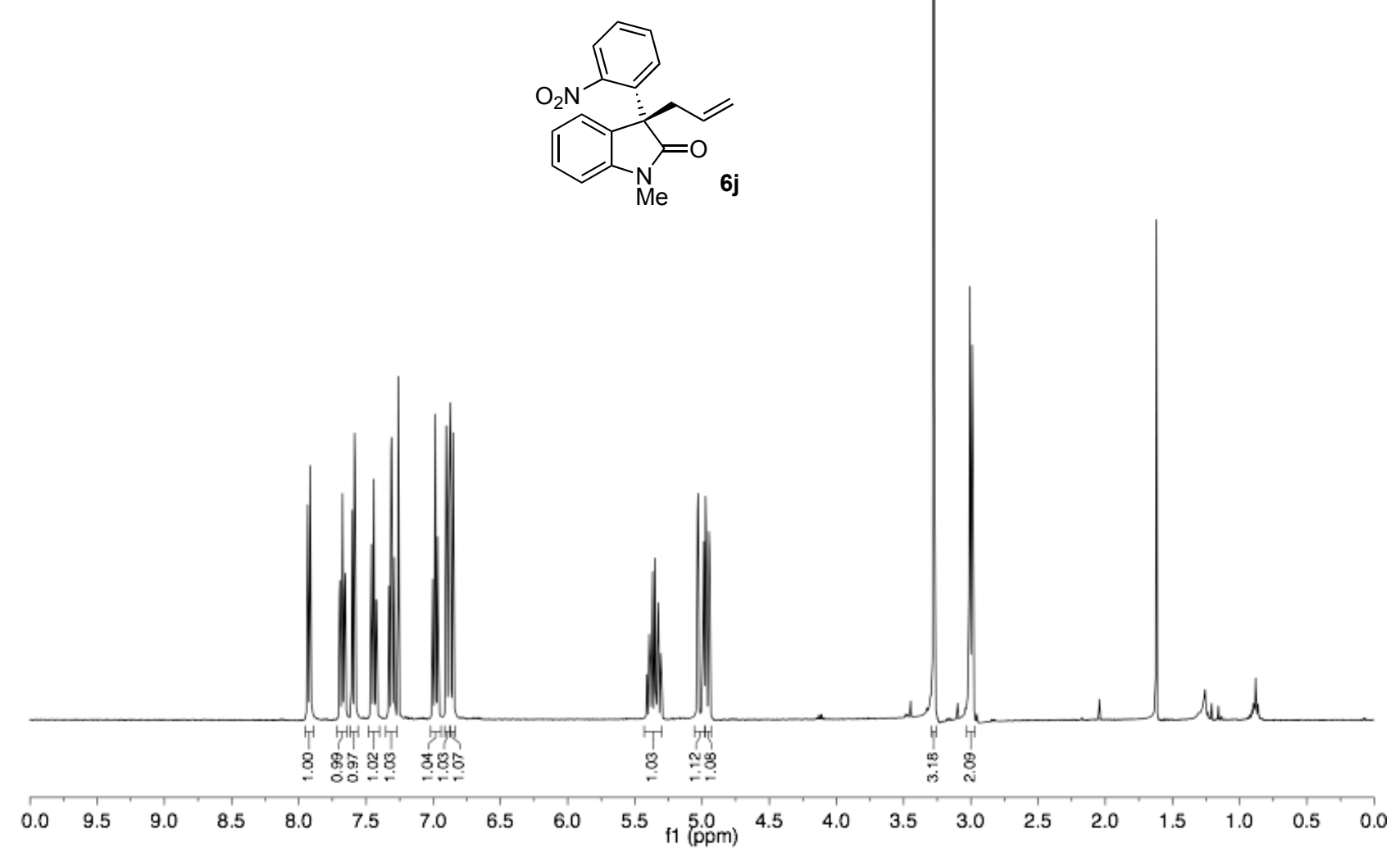

$\mathrm{CDCl}_{3}, 400 \mathrm{MHz}$
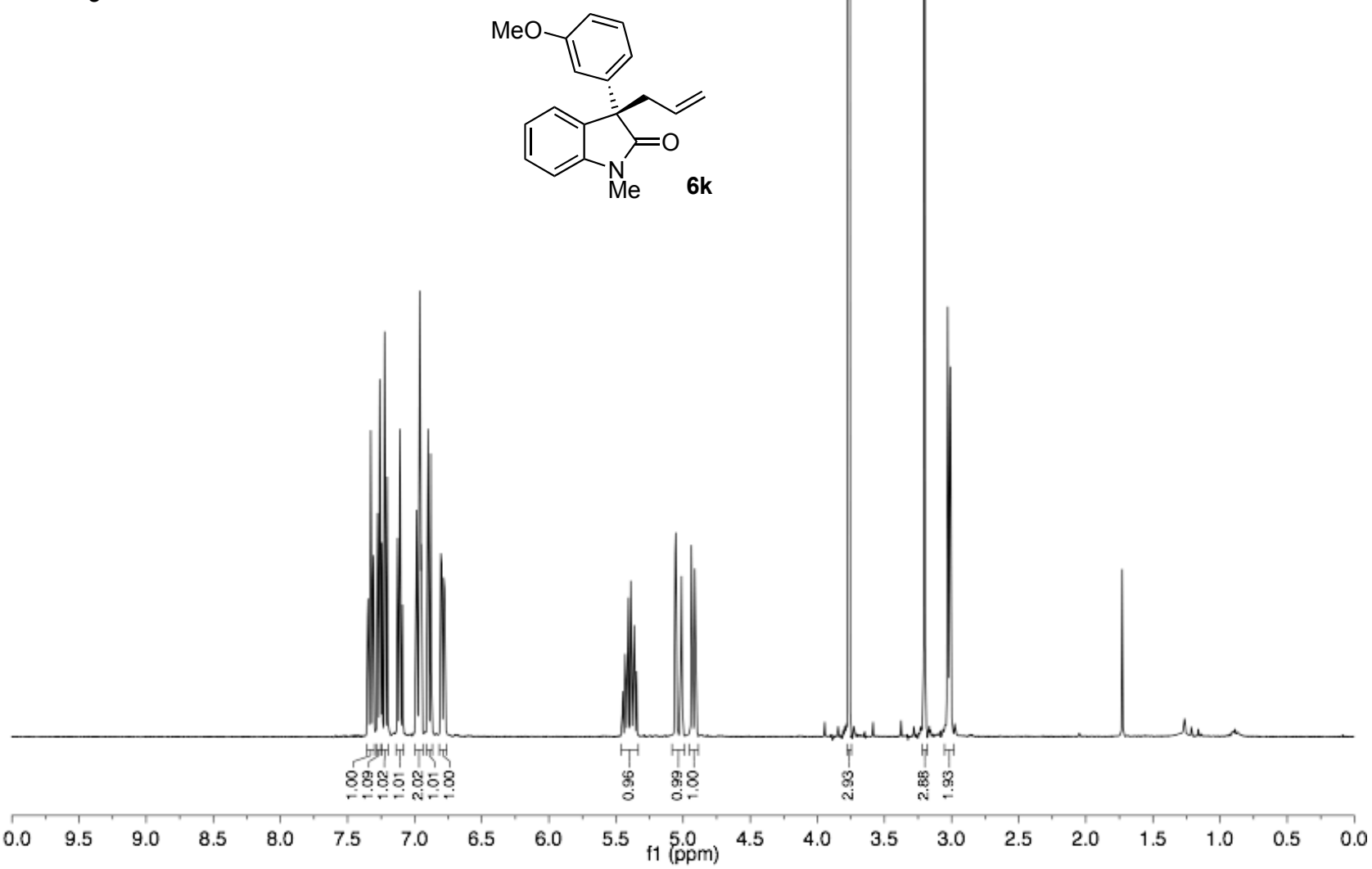